Fabio Bobrow

Controle de um pêndulo invertido com 6 graus de liberdade e rodas de reação 

Fabio Bobrow

\section{Controle de um pêndulo invertido com 6 graus de liberdade e rodas de reação}

Dissertação apresentada à Escola Politécnica da Universidade de São Paulo para obtenção do título de Mestre em Engenharia Elétrica com ênfase em Automação e Controle.

Orientador: Prof. Dr. Paulo Sérgio Pereira da Silva

\section{São Paulo}

2015 
Este exemplar foi revisado e corrigido em relação à versão original, sob responsabilidade única do autor e com a anuência de seu orientador.

São Paulo de de

Assinatura do autor:

Assinatura do orientador:

Catalogação-na-publicação

Bobrow, Fabio

Controle de um pêndulo invertido com 6 graus de liberdade e rodas de reação / F. Bobrow -- versão corr. -- São Paulo, 2015.

$105 \mathrm{p}$.

Dissertação (Mestrado) - Escola Politécnica da Universidade de São Paulo. Departamento de Engenharia de Telecomunicações e Controle.

1.SISTEMAS DE CONTROLE 2.CONTROLE MULTIVARIACIONAL 3.SISTEMAS NÃO LINEARES I.Universidade de São Paulo. Escola Politécnica. Departamento de Engenharia de Telecomunicações e Controle II.t. 
Nesta dissertação, um tipo diferente de pêndulo invertido controlado por rodas de reação é apresentado. Sua principal diferença está em seu ponto de articulação, que é constituído por uma junta esférica que permite com que o pêndulo gire em torno de seus três eixos. Além disso, três rodas de reação são utilizadas para seu controle e estabilização.

Primeiramente, um modelo do sistema é obtido a partir das equação de EulerLagrange, das leis de Newton e das leis de Kirchhoff. Em seguida, uma lei de controle que assegura a estabilização assintótica do sistema em um grande domínio é proposta. Por fim, simulações são realizadas para validar o controlador projetado.

Esse sistema possui diversas características interessantes, tanto do ponto de vista teórico como do ponto de vista de pesquisa. Do ponto de vista teórico, o sistema é nãolinear e suas entradas são fortemente acopladas, o que torna particularmente adequado para o processo de projeto e implementação de diversas técnicas de estabilização. Do ponto de vista de pesquisa, são consideradas duas técnicas de controle não linear: linearização padrão e linearização exata. Para que o sistema seja robusto e não desperdice energia, essas duas leis de controle diferentes são comutadas para a obtencão de um número suficiente de domínio de estabilidade.

Palavras-chave: pêndulo invertido, rodas de reação, sistema não-linear, linearização padrão, linearização exata 


\section{Abstract}

In this dissertation, a different kind of the reaction wheel pendulum is presented. The main difference is that its articulation point consists of a ball joint that allows the pendulum to rotate around its three axes. Furthermore, three reaction wheels are used for its control and stabilization.

First, a model of the system is obtained from Euler-Lagrange equations, Newton laws and Kirchhoff laws. After that, a control law that assure asymptotic stabilization of the system in a large domain is proposed. Finally, simulations are performed to validate the designed controller.

This system has several interesting features, both from a theoretical standpoint as from a research standpoint. From a theoretical standpoint, the system is nonlinear and its inputs are tightly coupled, making it particularly suitable for the design and implementation process of various stabilization techniques. From a research standpoint, two non-linear control techniques are considered: standard linearization and exact linearization. For the system to be robust and do not waste energy, these two different control laws are switched for obtaining a sufficiently large domain of stability.

Keywords: inverted pendulum, reaction wheel, nonlinear system, standard linearization, exact linarization 


\section{Lista de Figuras}

1.1 Exemplos de robôs capazes de caminhar ${ }^{1} \ldots \ldots \ldots \ldots$

1.2 Veículos e robôs auto-balanceáveis ${ }^{2} \ldots \ldots \ldots \ldots \ldots$

1.3 Pêndulo invertido Cart-pole $\ldots \ldots \ldots \ldots \ldots \ldots$

1.4 Outros tipos de pêndulos invertidos ${ }^{4} \ldots \ldots \ldots \ldots$

1.5 Pêndulo invertido controlado por rodas de reação $0^{5} \ldots \ldots \ldots$

1.6 Roda de reação ${ }^{6} \ldots \ldots \ldots \ldots$. . . . . . . . . . . . . . 7

2.1 Dinâmica do sistema completo . . . . . . . . . . . . . . . . 11

2.2 Diagrama de blocos do sistema completo . . . . . . . . . . . . . . 12

2.3 Diagrama de blocos do sub-sistema da haste . . . . . . . . . . . . . 13

2.4 Dinâmica do sub-sistema da haste . . . . . . . . . . . . . . . . . . 13

2.5 Notação utilizada nos ângulos de Euler . . . . . . . . . . . . . . . . . . . 14

2.6 Notação utilizada nos ângulos de Bryant . . . . . . . . . . . . . . . . 15

2.7 Diagrama de blocos do sub-sistema das rodas de reação $\ldots . .20 .20$

2.8 Dinâmica do sub-sistema das rodas de reação . . . . . . . . . . . . . . 21

2.9 Diagrama de blocos do sub-sistema dos motores elétricos . . . . . . . . . 22

2.10 Dinâmica do sub-sistema dos motores elétricos . . . . . . . . . . . 22

2.11 Dimensões de cilindros . . . . . . . . . . . . . . . . . . . . . 24

3.1 Diagrama de blocos do sistema completo . . . . . . . . . . . . . . 29

3.2 Diagrama de blocos do sistema completo e considerado (haste) . . . . 30

3.3 Diagrama de blocos do sub-sistema da haste . . . . . . . . . . . . . 33

3.4 Diagrama de blocos do sub-sistema da haste linearizado (linearização padrão . . . . . . . . . . . . . . . . . . 35

3.5 Diagrama de blocos do sub-sistema da haste linearizado (linearização padrão) e simplificado . . . . . . . . . . . . . . . . 36

3.6 Pólos e zeros de transmissão do sistema considerado (haste) . . . . . . . 37

3.7 Diagrama de blocos do sistema completo e considerado (haste e rodas de reação $\ldots \ldots \ldots$. . . . . . . . . . . . . . . . . . 39

3.8 Diagrama de blocos do sub-sistema das rodas de reação . . . . . . . . . 40

3.9 Diagrama de blocos do sistema considerado (haste e rodas de reação) . . 41

3.10 Diagrama de blocos simplificado do sistema considerado (haste e rodas

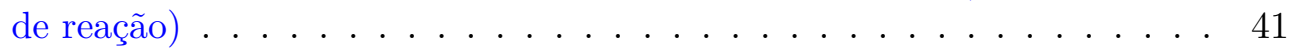


3.11 Pólos e zeros de transmissão do sistema considerado (haste e rodas de reação) . . . . . . . . . . . . . . . . . . 43

3.12 Diagrama de blocos do sistema completo e considerado (haste, rodas de reação e motores elétricos) . . . . . . . . . . . . . . . . . . 44

3.13 Diagrama de blocos do sub-sistema dos motores elétricos . . . . . . . . . 46

3.14 Diagrama de blocos do sistema considerado (haste, rodas de reação e motores elétricos) . . . . . . . . . . . . . . . . 46

3.15 Diagrama de blocos simplificado do sistema considerado (haste, rodas de reação e motores elétricos) . . . . . . . . . . . . . . . . . . 47

3.16 Pólos e zeros de transmissão do sistema considerado (haste, rodas de reação e motores elétricos) . . . . . . . . . . . . . . . . . . . . . . 49

3.17 Pólos e zeros de transmissão do sistema considerado (haste, rodas de reação e motores elétricos) 2 . . . . . . . . . . . . . . . . . . 50

3.18 Diagrama de blocos desacoplado do sistema considerado (haste, rodas de reação e motores elétricos) . . . . . . . . . . . . . . . 52

4.1 Diagrama de blocos do sistema composto pela haste com realimentação linearizante ...................... 53

4.2 Diagrama de blocos do sistema composto pela haste com realimentação linearizante e realimentação de estados . . . . . . . . . . . . . . . . . . 54

4.3 Diagrama de blocos do sistema composto pela haste com linearização padrão e realimentação de estados . . . . . . . . . . . . . . . . 54

4.4 Diagrama de blocos do sistema composto pela haste e rodas de reação com realimentação linearizante, realimentação de estados da haste e realimentação de estados das rodas de reação desprezando o coeficiente de atrito das rodas . . . . . . . . . . . . . . . . . . .

4.5 Diagrama de blocos do sistema composto pela haste com linearização padrão e rodas de reação com realimentação de estados da haste e realimentação de estados das rodas de reação desprezando o coeficiente de atrito das rodas . . . . . . . . . . . . . . . . . .

4.6 Diagrama de blocos do sistema composto pela haste e rodas de reação com realimentação linearizante da haste, realimentação de estados da haste e realimentação de estados das rodas de reação . . . . . . . . . . . 56

4.7 Diagrama de blocos do sistema composto pela haste, rodas de reação e motores elétricos com realimentação linearizante da haste, realimentação de estados da haste, realimentação de estados das rodas de reação e compensador de ganhos dos motores elétricos desprezando a realimentação dos estados das rodas nos motores . . . . . . . . . . . . . . 56

4.8 Diagrama de blocos do sistema composto pela haste, rodas de reação e motores elétricos com realimentação linearizante da haste, realimentação de estados da haste, realimentação de estados das rodas de reação e compensador de ganhos dos motores elétricos . . . . . . . . . . . 57

4.9 Diagrama de blocos do sub-sistema da haste com realimentação linearizante 58 
4.10 Diagrama de blocos do sub-sistema da haste linearizado (linearização exata . . . . . . . . . . . . . . . . . . . 5 59

4.11 Diagrama de blocos do sub-sistema da haste linearizado (linearização exata) com realimentação de estados . . . . . . . . . . . . . . 60

4.12 Diagrama de blocos do sub-sistema da haste linearizado (linearização exata) com realimentação de estados simplificado . . . . . . . . . . . 61

4.13 Pólos e zeros de transmissão do sistema com a haste em malha fechada . 63

4.14 Resultados da simulação 1 . . . . . . . . . . . . . . . . . . . . 64

4.15 Diagrama de blocos do sub-sistema da haste linearizado (linearização exata) com realimentação de estados e erros de medição . . . . . . . . . 65

4.16 Resultados da simulação $2 \ldots$. . . . . . . . . . . . . . . 66

4.17 Diagrama de blocos do sistema considerado (haste) com realimentação de estados desacoplado . . . . . . . . . . . . . . . . 67

4.18 Diagrama de blocos do sistema considerado (haste e rodas de reação) com realimentação de estados desacoplado . . . . . . . . . . . . . 68

4.19 Diagrama de blocos do sistema considerado (haste e rodas de reação) com realimentação de estados desacoplado desprezando o coeficiente de atrito das rodas . . . . . . . . . . . . . . . . . . . 6 68

4.20 Resultado da simulação $3 \ldots \ldots$. . . . . . . . . . . . . . . . 72

4.21 Resultado da simulação 4 . . . . . . . . . . . . . . . . . 73

4.22 Resultado da simulação 5 . . . . . . . . . . . . . . . . . . . . 74

4.23 Resultado da simulação 6 . . . . . . . . . . . . . . . . . 75

4.24 Diagrama de blocos do sistema considerado (haste, rodas de reação e motores elétricos) com realimentação de estados desacoplado . . . . . . 76

4.25 Diagrama de blocos do sistema considerado (haste, rodas de reação e motores elétricos) com realimentação de estados desacoplado desprezando a realimentação dos estados das rodas nos motores . . . . . . . . . 76

4.26 Resultado da simulação $7 \ldots \ldots$. . . . . . . . . . . . . 78

4.27 Resultado da simulação 8 . . . . . . . . . . . . . . . . . . 79

4.28 Diagrama de blocos do sistema composto pela haste, rodas de reação e motores elétricos com realimentação linearizante da haste, realimentação de estados da haste, realimentação de estados das rodas de reação e compensador de ganhos dos motores elétricos . . . . . . . . . . 80

4.29 Resultado da simulação $9 \ldots \ldots$. . . . . . . . . . . . . . . . 81

4.30 Resultado da simulação $10 \ldots$. . . . . . . . . . . . . . . . 82

4.31 Comparação das entradas nas simulações com e sem a realimentação linearizante . . . . . . . . . . . . . . . 83

4.32 Comparação das saídas nas simulações com e sem a realimentação linearizante . . . . . . . . . . . . . . . . 84 


\section{Lista de Tabelas}

2.1 Parâmetros do sub-sistema da haste . . . . . . . . . . . . . 13

2.2 Parâmetros do sub-sistema da roda de reação . . . . . . . . . . . . . . 21

2.3 Parâmetros do sub-sistema do motor elétrico . . . . . . . . . . . . . 23

2.4 Parâmetros adotados para o sistema . . . . . . . . . . . . 27

4.1 Tabela de Routh . . . . . . . . . . . . . . . . . . . . 70 


\section{Sumário}

1 Introdução 1

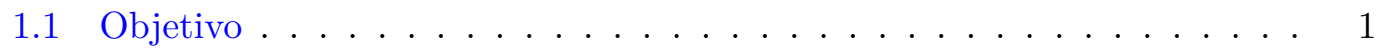

1.2 Justificativa . . . . . . . . . . . . . . . . . . . 1

1.3 Revisão Bibliográfica . . . . . . . . . . . . . . . . . . . 2

1.3.1 Robôs bípedes . . . . . . . . . . . . . . . . 2

1.3.2 Pêndulos invertidos . . . . . . . . . . . . . . . . . 3

1.3 .3 Rodas de reação . . . . . . . . . . . . . . . . . . . 7

1.3.4 Controle não-linear . . . . . . . . . . . . . . . . . . . 8

2 Modelagem 11

2.1 Definição do sistema . . . . . . . . . . . . . . . . . . . . 11

2.2 Modelagem da haste . . . . . . . . . . . . . . . . . . . . . . . 12

2.2.1 Modelagem pelas equações de Euler-Lagrange . . . . . . . . . . . 14

2.3 Modelagem das rodas de reação . . . . . . . . . . . . . . . . . . . 20

2.3.1 Modelagem pelas leis de Newton . . . . . . . . . . . . . 21

2.4 Modelagem dos motores elétricos . . . . . . . . . . . . . . . . 22

2.4.1 Modelagem pelas leis de Kirchhoff . . . . . . . . . . . . . . 23

2.5 Definição dos parâmetros . . . . . . . . . . . . . . . . 24

3 Análise $\quad 29$

3.1 Definição da sequência de análise . . . . . . . . . . . . . . . . . . 29

3.2 Análise da haste . . . . . . . . . . . . . . . . . . 30

3.2 .1 Sub-sistema da haste . . . . . . . . . . . . . . . 30

3.2 .2 Estabilidade. . . . . . . . . . . . . . . . . 36

3.2.3 Controlabilidade e observabilidade . . . . . . . . . . . . 37

3.3 Análise da haste com as rodas de reação . . . . . . . . . . . . . . . 38

3.3.1 Sub-sistema das rodas de reação . . . . . . . . . . . . . . . 39

3.3.2 Inclusão das rodas de reação no sistema . . . . . . . . . . . . . . 40

3.3 .3 Estabilidade . . . . . . . . . . . . . . . . . . . . 42

3.3.4 Controlabilidade e observabilidade . . . . . . . . . . . . 43

3.4 Análise da haste com as rodas de reação e motores elétricos . . . . . . . 44

3.4.1 Sub-sistema dos motores elétricos . . . . . . . . . . . . . . 44

3.4.2 Inclusão dos motores elétricos no sistema . . . . . . . . . . . 46 
3.4 .3 Estabilidade. . . . . . . . . . . . . . . . . . 48

3.4.4 Controlabilidade e observabilidade . . . . . . . . . . . . 50

3.5 Desacoplamento de canais . . . . . . . . . . . . . . . . . . . . 51

4 Controle 53

4.1 Definição da técnica de controle . . . . . . . . . . . . . . . 53

4.2 Controle da haste . . . . . . . . . . . . . . . . . . . 57

4.2 .1 Realimentação linearizante . . . . . . . . . . . . . . 57

4.2 .2 Realimentação de estados . . . . . . . . . . . . . . . . . . . . 59

4.2 .3 Estabilidade. . . . . . . . . . . . . . . . . . . . 62

4.2 .4 Simulações . . . . . . . . . . . . . . . . . . . . . 63

4.3 Controle da haste com as rodas de reação . . . . . . . . . . . . . . . 67

4.3.1 Critério de estabilidade de Routh . . . . . . . . . . . . . . . . 69

4.3 .2 Simulações . . . . . . . . . . . . . . . . . . . . . 71

4.4 Controle da haste com as rodas de reação e motores elétricos . . . . . . 75

4.4.1 Compensador de ganhos . . . . . . . . . . . . . . . . 76

4.4 .2 Simulações . . . . . . . . . . . . . . . . . . . . 77

4.5 Controlador final obtido . . . . . . . . . . . . . . . . . . 79

4.5.1 Simulações . . . . . . . . . . . . . . . . . . . 80

5 Conclusão $\quad 85$

$\begin{array}{lr}\text { Referências Bibliográficas } & 87\end{array}$

$\begin{array}{lr}\text { A Códigos do MATLAB } & 89\end{array}$ 


\section{Capítulo 1}

\section{Introdução}

Robôs bípedes são muitas vezes modelados com base em diversas versões de pêndulos invertidos. Esses modelos simplificados têm sido muito úteis para a análise e controle da marcha humanóide. Entretanto, os modelos de pêndulos invertidos encontrados na literatura não incluem os movimentos dos membros individuais e concentram-se apenas em dois pontos: o centro de pressão e o centro de massa. (Pons, 2008)

Ao representar todo o corpo humanóide apenas como um ponto de massa não levamos em consideração os momentos de inércia dos braços e tronco, componentes fundamentais para o movimento humanóide e, principalmente, seu equilíbrio. A fim de preencher essa lacuna, será analisado um tipo diferente de pêndulo invertido no estudo de equilíbrio de robôs bípedes. (Sheng-Jun and Hong-Xu, 2009)

\section{$1.1 \quad$ Objetivo}

O objetivo deste trabalho é obter um modelo aderente e robusto para um pêndulo invertido com seis graus de liberdade controlado por rodas de reação e desenvolver uma técnica de controle não-linear sólida para estabilização do mesmo.

O estudo também envolve o desenvolvimento de um simulador dinâmico ${ }^{1}$ para validação e refinamento da técnica de controle.

\subsection{Justificativa}

Pêndulos invertidos são comumente utilizados como uma maneira de explorar sistemas mecatrônicos não-lineares com dinâmica rápida. Este tipo de estudo é particularmente adequado para o processo de aprendizagem na área de controle, a fim de projetar e implementar diversas técnicas de estabilização.

Existem diversos esquemas possíveis para um sistema de pêndulo invertido, cada um com um modelo matemático distinto. Um pêndulo invertido controlado por rodas de reação é talvez um dos tipos mais simples de sistema em termos de suas propriedades

${ }^{1}$ MATLAB e Simulink. 
dinâmicas. Ao mesmo tempo, esse tipo de pêndulo apresenta diversas propriedades, tais como subatuação ${ }^{2}$ e não-linearidade, que o tornam um sistema atraente e útil para a investigação e formação avançada. (Friedland, 2005)

Além disso, o pêndulo de estudado neste trabalho, que possui seis graus de liberdade (ao invés de dois do clássico pêndulo invertido controlado por rodas de reação), é pouco presente na literatura atual devido a sua alta complexidade.

\subsection{Revisão Bibliográfica}

Para um melhor entendimento dos tópicos relactionados à modelagem, análise e controle do sistema, os principais conceitos envolvidos nesse trabalho serão definidos, a saber: robôs bípedes, pêndulos invertidos, rodas de reação e controle não-linear.

\subsubsection{Robôs bípedes}

O estudo de robôs bípedes, além de particularmente desafiador, tem sido motivado pelas suas aplicações potenciais na locomoção em terrenos irregulares ou descontínuos, tais como em degraus de uma escada. Além disso, outras aplicações incluem a possibilidade destes robôs substituirem seres humanos em tarefas perigosas ou até mesmo serão utilizados na reabilitação de pessoas.

Existem muitos trabalhos sobre o caminhar bípede, assunto que foi de interesse até mesmo a Aristóteles ao questionar "por que homens e pássaros são bípedes enquanto os peixes não possuem pés?" (Aristotle, 1984). De volta ao nosso tempo, existem centenas de robôs capazes de caminhar, construídos por laboratórios de pesquisa, universidades e grandes empresas. O RABBIT (Figura 1.1a) é um exemplo de robô bípede construído pelo Centre National de la Recherche Scientifique (CNRS) e pelo Conselho Nacional de Pesquisa da França. O STriDER (Figura 1.1b) é um robô de três pernas construído pelo Laboratório de Robótica e Mecânica de Virginea Polytechnic Institute and State University (Virginia Tech), apenas para enumerar alguns.

\footnotetext{
${ }^{2}$ Subatuação é um termo técnico usado em robótica e teoria de controle para descrever sistemas mecânicos que não podem ser comandados a seguir trajetórias arbitrárias no espaço.
} 


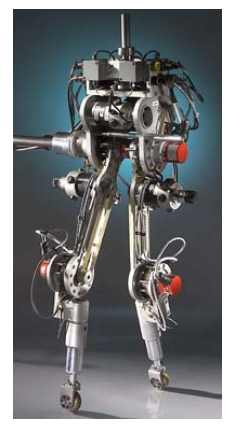

(a) Robô RABBIT

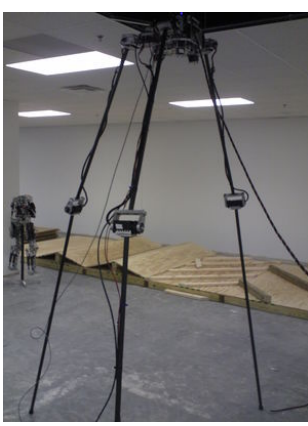

(b) Robô STriDER

Figura 1.1: Exemplos de robôs capazes de caminhar ${ }^{3}$

Apesar de existirem inúmeras aplicações práticas, existe também uma grande deficiência na teoria fundamental de controle de robôs bípedes (ao menos quando comparamos com o grande número de protótipos construídos). Até as aplicações mais avançadas se baseiam fortemente em resultados heurísticos e princípios como o Zero Moment Point (ZMP) que não garantem estabilidade. Um resultado disso é que, normalmente, apenas baixas velocidades são alcançadas. (Morazzani and Ren, 2008)

\subsubsection{Pêndulos invertidos}

Levando o nome do Latin pendere, que significa pendurar ${ }^{4}$, o pêndulo é um dos exemplos mais importantes de controle dinâmico e tem sido estudado extensivamente desde o tempo de Galileo (Galilei, 1957). De fato, o estudo empírico do movimento do pêndulo de Galileo levantou questões importantes na mecânica que foram respondidas apenas depois de algumas décadas com a formulação das leis de movimento de Newton e o trabalho de outros pesquisadores.

Durante seus experimentos, Galileo observou que um pêndulo quase retorna a sua altura de lançamento e eventualmente vem a repousar, onde os pêndulos mais leves tendem a repousar mais rapidamente. Ele descobriu que o período de oscilação do pêndulo independe de seu peso, depende apenas de seu comprimento e que o período independe de sua amplitude para pequenas amplitudes. Todas essas propriedades, hoje em dia, são facilmente derivadas através das leis de Newton e equações de movimento.

Em cursos de física, o pêndulo simples é muitas vezes apresentado para ilustrar conceitos básicos de movimento periódico e conservação de energia, enquanto que conceitos mais avançados, como o movimento caótico, são ilustrados com o pêndulo forçado e/ou o duplo pêndulo. (Block and Spong, 2007)

Considerando um pêndulo simples onde $\theta$ é o ângulo que o pêndulo faz com a vertical, $l$ é seu comprimento, $m$ sua massa e $g$ a aceleração da gravidade ${ }^{5}$, a equação

\footnotetext{
${ }^{3}$ Fonte: http://www.eecs.umich.edu/ qrizzle/papers/PhotosRABBIT/Rabbit_tapis.jpg / http: //www.romela.org/wp-content/uploads/2015/02/smaler_1-768×1024.jpg

${ }^{4}$ Outros cognatos incluem "suspender" e "depender"

${ }^{5} 9,8 \mathrm{~m} / \mathrm{s}^{2}$ na superfície da terra.
} 
do movimento (do pêndulo simples) é dada por:

$$
\ddot{\theta}+\frac{g}{l} \operatorname{sen} \theta=0
$$

Note que a Equação (1.1) é uma equação diferencial ordinária não linear devido ao termo $\operatorname{sen} \theta$. Ao aproximar o termo $\operatorname{sen} \theta$ a $\theta$, o que é válido apenas para pequenos valores de $\theta$, é obtido o seguinte sistema linear:

$$
\ddot{\theta}+\frac{g}{l} \theta=0
$$

A Equação (1.2) é chamada de equação do oscilador harmônico simples. Sua solução geral é dada por:

$$
\theta(t)=A \cos (\omega t)+B \operatorname{sen}(\omega t)
$$

Onde $A$ e $B$ são constantes determinadas pelas condições iniciais e $\omega$ é uma constante determinada pelas características do pêndulo dadas por:

$$
\begin{aligned}
A & =\theta(0) \\
B & =\frac{\dot{\theta}(0)}{\omega} \\
\omega & =\sqrt{\frac{g}{l}}
\end{aligned}
$$

O pêndulo simples é interessante para estudar problemas de dinâmica e controle. É ideal para estudantes universitários de praticamente todos os níveis, desde calouros da graduação até veteranos da pós-graduação. No entanto, a sua importância é muito mais do que acadêmica, pois diversos sistemas práticos de engenharia podem ser modelados de forma aproximada como pêndulos.

Em aeronáutica, o pêndulo é muitas vezes utilizado para modelar o lançamento de um foguete. A fim de manter a atitude adequada durante a subida, um controle ativo torna-se necessário. O ângulo de ataque do foguete pode ser controlado variando o ângulo de saída de seus propulsores. Nesse caso, a dinâmica do foguete pode ser aproximada a de um pêndulo invertido.

Em biomecânica, o pêndulo é muitas vezes utilizado para modelar a marcha bípede. Em robôs bípedes a perna de suporte em contato com o solo é aproximada a um pêndulo invertido, enquanto que a perna de balanço é aproximada a um pêndulo simples suspenso a partir do quadril. De fato, os estudos de dinâmica postural humana e locomotiva têm encontrado muitas semelhanças com a dinâmica dos pêndulos. Medições de atividade muscular durante a caminhada indicam que os músculos das pernas são ativos principalmente durante o início e o fim da fase de balanço, o que permitem que a perna balance como um pêndulo. Assim a natureza tem nos ensinado a explorar a tendência natural da perna de balançar como um pêndulo durante a caminhada, o que 
pode explicar em parte a eficiência energética do caminhar. Da mesma forma, a posição de relaxamento em pé requer um controle de equilíbrio. A chamada oscilação postural é resultado de reflexos de estiramento dos músculos, que basicamente é um tipo de estabilização por realimentação de um pêndulo invertido. (Goswami, 2008)

Em robótica, o transportador humano Segway (Figura 1.2a) é uma invenção recente que conseguiu um sucesso comercial. O Segway é de fato um pêndulo invertido controlado. Com base em informações sensoriais de giroscópio montados na base do equipamento, um sistema de controle computadorizado mantém o equilíbrio conforme o ser-humano se locomove com ele. Há também robôs autônomos de duas rodas autobalanceáveis (Figura 1.2b), destinados a manter o equilíbrio e locomoção sem a intervenção humana. Tais sistemas foram desenvolvidos e contruídos em diversos laboratórios de pesquisa ao redor do mundo que visavam investigar questões de controle, navegação autônoma, coordenação de grupos, entre outras. (Craig, 2005)

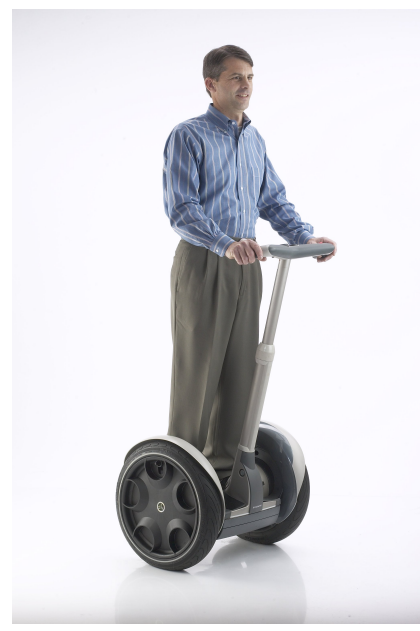

(a) Segway

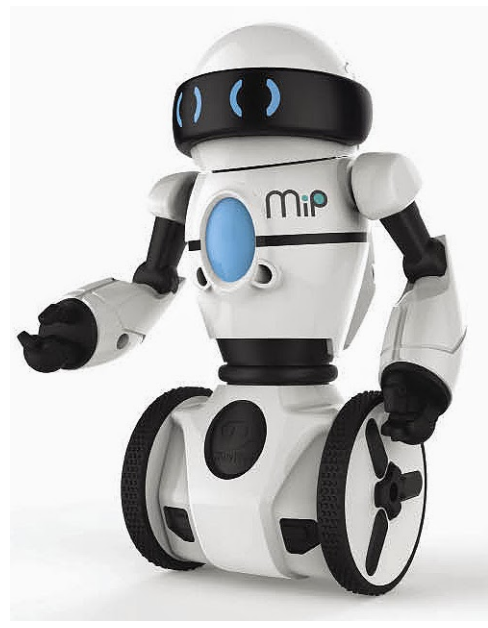

(b) Robô autônomo

Figura 1.2: Veículos e robôs auto-balanceáveis ${ }^{6}$

Há diversos exemplos de problemas de sistemas de controle em engenharia onde a dinâmica do pêndulo fornece informações úteis, incluindo a estabilização de guindastes, estabilização longitudinal de navios e caminhões, dentre outros. Assim, um estudo dos sistemas pendulares é um excelente ponto de partida para entender as questões de dinâmica e controle não lineares.

Recentemente, tem surgido vários dispositivos utilizados para ilustrar a dinâmica do pêndulo invertido e para facilitar a concepção e execução de sistemas de controle. A mais antiga dessas idéias é o chamado sistema Cart-pole (Figura 1.3). Nesse sistema o ponto de articulação do pêndulo é deslocado de forma linear a fim de controlar o movimento pendular.

\footnotetext{
${ }^{6}$ Fonte: https://wehse.files.wordpress.com/2012/08/man_on_iseries2.jpg / http://www. mechatronictips . com/wp-content/uploads/2013/11/MIP-WowWee-robot.jpg
} 


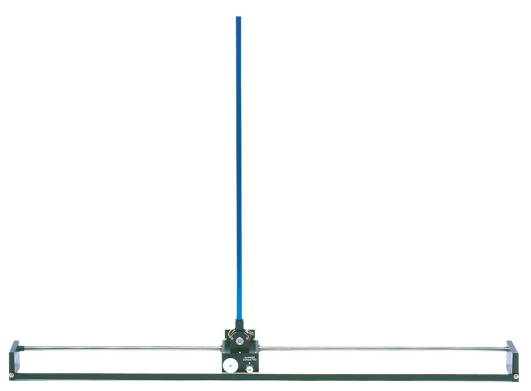

Figura 1.3: Pêndulo invertido Cart-pole

Inovações posteriores dessa idéia foram o Pendubot (Figura 1.4a) e o Furuta (Figura 1.4b). Em ambos dispositivos, o segundo ligamento é um pêndulo invertido cujo movimento pendular é controlado por um movimento de rotação (em vez de um movimento linear) do primeiro ligamento. O Pendubot é concebido de modo que os eixos de rotação dos dois ligamentos são paralelos, enquanto que no Furuta esses eixos de rotação são perpendiculares.

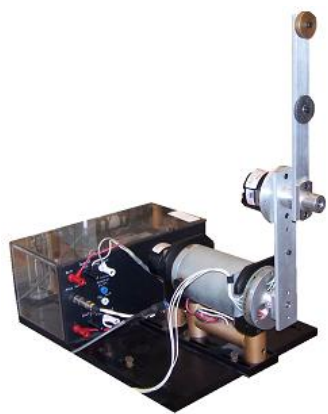

(a) Pendubot

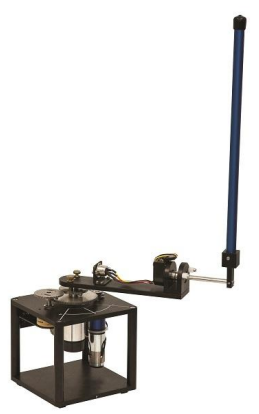

(b) Furuta

Figura 1.4: Outros tipos de pêndulos invertidos ${ }^{8}$

O pêndulo invertido controlado por rodas de reação (Figura 1.5) é o mais recente desses dispositivos. Nesse dispositivo, o ponto de articulação do pêndulo é livre, enquanto que na outra extremidade do pêndulo encontra-se uma roda de reação impulsionada por um atuador. As variações de velocidade na roda geram um torque que controlam o movimento pendular. (Seman and Juhás, 2011)

\footnotetext{
${ }^{7}$ Fonte: http://www. dofware.com/img/Linear-Inverted-Pendulum-IP02.jpg

${ }^{8}$ Fonte: http://www.quanser.com/Images/Products/496-800-600.jpg / http://coecsl.ece. illinois . edu/images/PendubotAllUp. JPG
} 


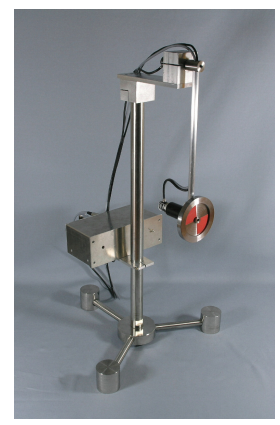

Figura 1.5: Pêndulo invertido controlado por rodas de reação ${ }^{9}$

\subsubsection{Rodas de reação}

Rodas de reação (Figura 1.6) formam uma classe de atuadores elétricos, presentes principalmente em satélites, que não exigem qualquer propulsor e são capazes de fornecer torques aos satélites. Seu princípio de funcionamento está baseado na conservação do momento angular. A atuação da roda de reação sobre o satélite se realiza por intercâmbio de momento angular, limitado ao eixo de rotação da roda. Devido à grande diferença entre a inércia do satélite e da roda de reação, um controle de atitude com muita precisão é possível com esse sistema. Rodas de reação são particularmente úteis quando o satélite deve ser rotacionado por quantidades muito pequenas, tais como para manter um telescópio ou uma câmera apontada para uma determinada direção. Rodas de reação só podem rotacionar um satélite em torno de seu centro de massa e não são capazes de movê-lo de um lugar a outro.

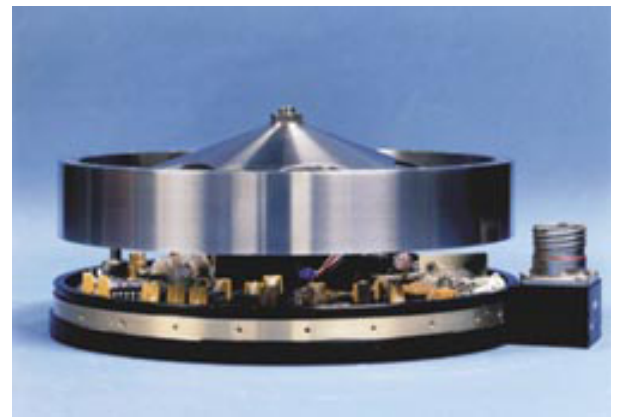

Figura 1.6: Roda de reação ${ }^{10}$

Rodas de reação são tipicamente constituídas de um motor elétrico, geralmente um motor sem escovas, um mancal e um elemento de inércia. $\mathrm{O}$ elemento de inércia e o motor são montados sobre um mancal que deve garantir a precisa rotação em torno de um eixo. A velocidade de rotação do sistema é controlada por um sistema eletrônico

\footnotetext{
${ }^{9}$ Fonte: http://www.pendcon.de/tI_files/RWP\%20web\%20site.jpg

${ }^{10}$ Fonte: http://www . algor.com/news_pub/cust_app/goddard/rxwheel.jpg
} 
de acionamento do motor. Rodas de reação podem ser comandadas de duas maneiras distintas: por rotação ou por torque. Quando comandadas por torque, as rodas de reação devem ser capazes de estimar o torque útil gerado por ele (o torque efetivo menos as perdas).

Apesar de inicialmente serem utilizadas única e exclusivamente para o controle de atitude de satélites, hoje em dia elas são utilizadas em diversos ramos, desde carros da fórmula 1 até kits acadêmicos. Conforme já foi dito, o conceito básico de uma roda de reação baseia-se na lei de conservação de momento angular $L$ :

$$
L=\sum_{i=1}^{n} J_{i} \omega_{i}
$$

Onde $J$ e $\omega$ são respectivamentes o momento de inércia e a velocidade angular do $i$-ésimo corpo que compõe o sistema. No caso de um pêndulo invertido controlado por rodas de reação, a roda apresenta uma massa giratória separada da massa do pêndulo (o qual também pode ser pensado como uma massa giratória), assim a expressão do momento angular $L$ deve conter os termos da roda de reação bem como do próprio pêndulo:

$$
L_{\text {sistema }}=J_{\text {pêndulo }} \omega_{\text {pêndulo }}+J_{\text {roda }} \omega_{\text {roda }}
$$

$\mathrm{Na}$ equação acima torna-se claro que para manter o momento angular do sistema constante é necessário um balanço entre a velocidade angular do pêndulo e a da roda. Pode-se notar também como a alteração da velocidade angular da roda tem ação direta na velocidade angular do pêndulo.

Um pêndulo invertido controlado por rodas de reação normalmente possui apenas dois graus de libertade, o ângulo que o pêndulo faz com a vertical e o ângulo que a roda de reação faz com a vertical. O sistma a ser estudado nesse trabalho é um pouco mais complexo por possuir uma junta esférica que proporciona três graus de libertade ao pêndulo. Dessa forma, são necessárias três rodas de reação para controlar o pêndulo, uma para cada eixo, totalizando assim seis graus de liberdade no sistema.

\subsubsection{Controle não-linear}

Sistemas de equações diferenciais ordinárias (ODEs) podem ser divididos em dois tipos: determinados e indeterminados. O último pode ser definido com um sistema de $m$ ODEs com $n$ variáveis dependentes, onde $n>m$. Sistemas deste tipo são normalmente estudados por engenheiros de controle. Em geral, são definidas $n-m$ das variáveis dependentes em função do tempo a fim de forçar o sistema a agir de uma maneira específica. Quando o sistema de equações diferenciais ordinárias é linear, existe um certo número de métodos para conceber facilmente o controle do sistema. Entretanto, quando o sistema de equações diferenciais ordinárias é não-linear, o controle é muito mais difícil e existem poucos métodos para solucioná-lo.

Um método que tem sido desenvolvido para conceber um controle mais fácil de sistemas não-lineares explora uma propriedade de tais sistemas conhecida como "Flat- 
ness" ou planitude que, segundo o dicionário Houaiss, é a propriedade de ser plano. Um sistema plano é um sistema de ODEs cujas curvas de solução estão em uma suave correspondêndia uma a uma com curvas arbitrárias, num espaço cuja dimensão é igual a $n-m$. Consequentemente, o encontro de soluções para esse tipo de sistemas é simplificado por encontrar as curvas em uma dimensão menor que satisfaçam as condições de contorno. (Isidori, 1995)

Um sistema é plano se existirem variáveis $y_{1}, \ldots, y_{n-m}$ dadas por uma equação da forma:

$$
y=h\left(t, x, \dot{x}, \ddot{x}, \ldots, x^{(k)}\right)
$$

De tal modo que as variáveis originais $x$ possam ser recuperadas a partir de $y$ (localmente) por uma equação da forma:

$$
x=g\left(t, y, \dot{y}, \ddot{y}, \ldots, y^{(l)}\right)
$$

As variáveis $y_{1}, \ldots, y_{n-m}$ são referidas como saídas planas. Quando $y$ é uma função do tempo e dos estados, o sistema é dito plano.

A geração de soluções que levam o sistema a partir de um estado inicial desejado para um valor final desejado é relativamente fácil. Considerando as condições iniciais e finais para os estados, suas derivadas e suas entradas, os valores para $y_{1}, \ldots, y_{n-m}$ e suas derivadas nos tempos iniciais e finais podem ser determinados. Em seguida, é preciso gerar funções do tempo que satisfaçam essas condições iniciais e finais. É interessante notar que a ligação de qualquer ponto de partida a um ponto de término do sistema é possível. Portanto, os sistemas planos são controláveis.

Ao conceber o controle para sistemas não-lineares, ocasionalmente é possível transformar os estados de tal maneira que o sistema de controle possa ser expresso de uma forma linear, conhecida também como linearização exata. Para esta classe de sistemas não-lineares, existe uma realimentação de estados (não-linear) que permite o cancelamento exato das não linearidades do sistema. Neste caso, o sistema em malha fechada torna-se linear, se ele for descrito em um sistema de coordenadas adequado.

Dessa forma, existem duas abordagens principais para o desenvolvimento de um projeto de controle para determinados sistemas não lineares: pode-se utilizar a linearização exata ou propriedade de "Flatness".

Não existem condições gerais que classifiquem um sistema em plano ou não. Além disso, não existem condições gerais para determinar as saídas planas de um sistema plano. No entanto, existem algumas propriedades específicas para sistemas planos. Todos os sistemas lineares controláveis ou que possuem uma linearização exata são planos. Sistemas Lagrangianos foram estudados em detalhe quanto à planitude e existem catálogos detalhando quais tipos de sistemas Lagrangianos são planos. Os resultados desse estudo revelam propriedades desses sistemas que indicam sua planitude. (Fliess and Rouchon, 1995)

Sistemas não-lineares que tem a propriedade de "Flatness" são relativamente fáceis de controlar. A determinação das saídas planas reque cálculos mais sofisticados. A 
informação estrutural contida nas equações de Lagrange costuma ser explorada para determinar se um sistema é plano e encontrar suas saídas planas. A geometria é utilizada na definição das saídas planas. 


\section{Capítulo 2}

\section{Modelagem}

O primeiro passo de qualquer problema de sistemas de controle consiste em deduzir um modelo matemático do sistema a ser controlado. Neste capítulo, é desenvolvido um modelo matemático que representa a dinâmica do pêndulo invertido e seus componentes.

\subsection{Definição do sistema}

O pêndulo invertido em estudo é composto, basicamente, por uma haste, três rodas de reação e três motores elétricos, conforme pode ser observado na Figura 2.1.

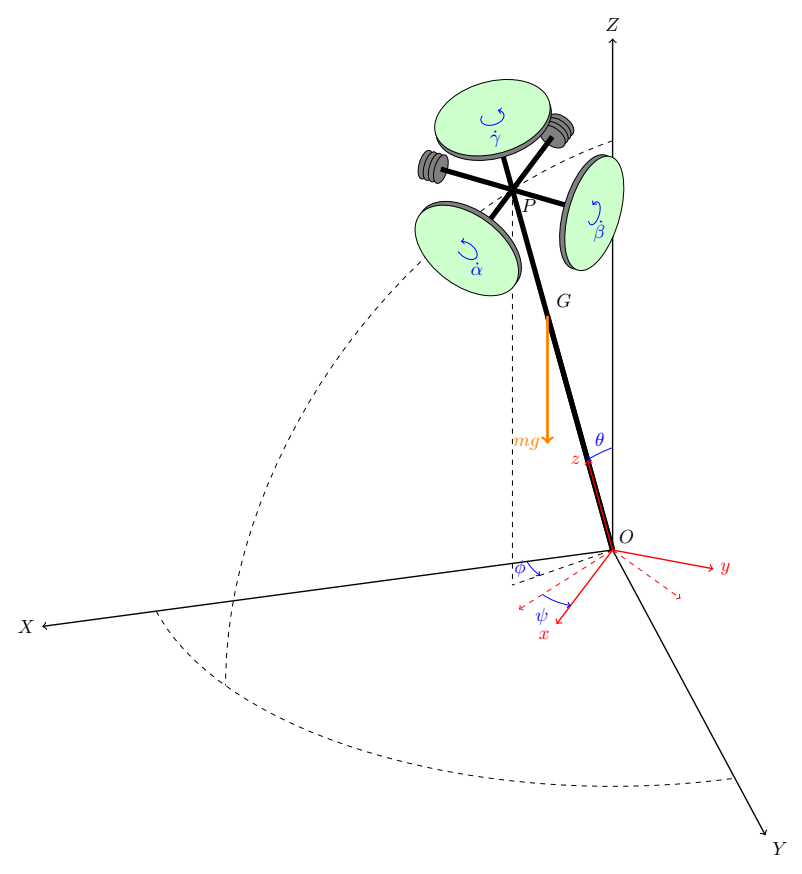

Figura 2.1: Dinâmica do sistema completo 
Em uma extremidade da haste (ponto $O$ ) escontra-se uma articulação esférica que proporciona três graus de liberdade ao sistema. Na extremidade oposta (ponto $P$ ) estão fixas três rodas de reação que possuem um grau de liberdade cada, totalizando assim seis graus de liberdade no sistema.

A posição e orientação do pêndulo é descrita pelas três coordenadas generalizadas $\phi, \theta$ e $\psi$ que correlacionam o sistema de coordenadas fixo no pêndulo $x y z$ e o sistema de coordenadas global $X Y Z$. O pêndulo possui massa $m$ e momentos de inércia $J_{x}, J_{y}$ e $J_{z}$. O centro de massa $G$ do pêndulo está a uma distância $z_{G}$ da articulação esférica, onde, devido a aceleração da gravidade $g$, é originada a força peso com sentido sempre vertical (eixo $Z$ ) e módulo $m g$.

As rodas de reação são impulsionadas por motores elétricos cujos eixos são ortogonais entre si. Os motores elétricos são acionados pelas tensões $e_{x}, e_{y}$ e $e_{z}$, quando ocorrem variações em suas velocidade surgem os torques $\bar{\tau}_{x}, \bar{\tau}_{y}$ e $\bar{\tau}_{z}$. Os responsáveis pelo controle da posição e da orientação do pêndulo, no entanto, são os torques úteis de reação $\tau_{x}, \tau_{y}$ e $\tau_{z}$, proporcionais ao momento de inércia das rodas $J_{r}$ e acelerações angulares $\ddot{\alpha}, \ddot{\beta}$ e $\ddot{\gamma}$ de cada roda. As rodas de reação possuem um contrapeso para manter o pêndulo simétrico.

Dessa forma, as entradas do sistema são as tensões nos motores elétricos $e_{x}, e_{y} \mathrm{e}$ $e_{z}$ e as saídas são as coordenadas generalizadas $\phi, \theta$ e $\psi$, que descrevem a posição e orientação do pêndulo.

A dinâmica do sistema do pêndulo pode ser entendida de modo mais fácil se for dividida em três sub-sistemas: motores elétricos, rodas de reação e haste (conforme a Figura 2.2).

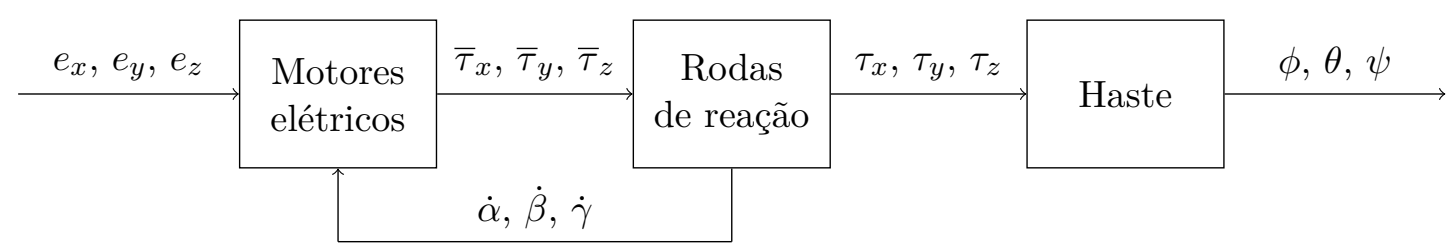

Figura 2.2: Diagrama de blocos do sistema completo

Cada sub-sistema possui sua dinâmica e será modelado individualmente a seguir. Entretanto, como o sub-sistema das rodas de reação influencia o sub-sistema dos motores elétricos (em outras palavras, o sub-sistema dos motores elétricos é realimentado), a modelagem foi feita de trás para frente: primeiro a haste, depois as rodas de reação e por fim os motores elétricos.

\section{$2.2 \quad$ Modelagem da haste}

O sub-sistema da haste possui como entrada os torques úteis de reação desenvolvidos pelas rodas de reação $\tau_{x}, \tau_{y}$ e $\tau_{z}$ e, como saída, os ângulos que descrevem a posição e orientação do pêndulo $\phi, \theta$ e $\psi$, conforme a Figura 2.3. 


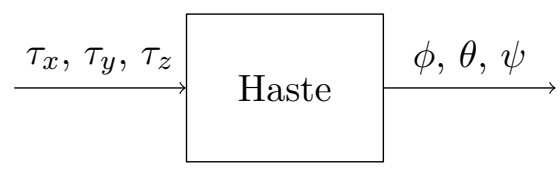

Figura 2.3: Diagrama de blocos do sub-sistema da haste

A dinâmica da haste pode ser observada na Figura 2.4 e seus parâmetros estão definidos na Tabela 2.1 .

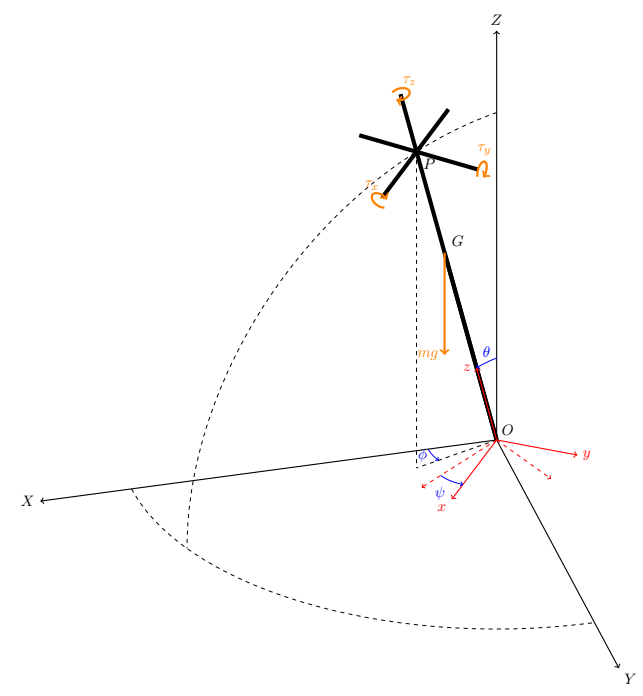

Figura 2.4: Dinâmica do sub-sistema da haste

Tabela 2.1: Parâmetros do sub-sistema da haste

\begin{tabular}{c|l} 
Símbolo & Parâmetro \\
\hline$\phi$ & Ângulo de Euler que descreve a posição e orientação do pêndulo \\
$\theta$ & Ângulo de Euler que descreve a posição e orientação do pêndulo \\
$\psi$ & Ângulo de Euler que descreve a posição e orientação do pêndulo \\
$m$ & Massa do pêndulo \\
$J_{x}$ & Momento de inércia do pêndulo em torno do eixo $x$ \\
$J_{y}$ & Momento de inércia do pêndulo em torno do eixo $y$ \\
$J_{z}$ & Momento de inércia do pêndulo em torno do eixo $z$ \\
$g$ & Aceleração da gravidade \\
$z_{G}$ & Distância do centro de massa $G$ do pêndulo a articulação esférica $O$ \\
$\tau_{x}$ & Torque útil de reação desenvolvido pela roda de reação do eixo $x$ \\
$\tau_{y}$ & Torque útil de reação desenvolvido pela roda de reação do eixo $y$ \\
$\tau_{z}$ & Torque útil de reação desenvolvido pela roda de reação do eixo $z$ \\
$x y z$ & Sistema de coordenadas fixo no pêndulo \\
$X Y Z$ & Sistema de coordenadas global
\end{tabular}




\subsubsection{Modelagem pelas equações de Euler-Lagrange}

A dinâmica da haste será modelada pelas equação de Euler-Lagrange. A equação geral de Euler-Lagrange é dada por:

$$
\frac{d}{d t}\left(\frac{\partial L}{\partial \dot{q}_{i}}\right)-\left(\frac{\partial L}{\partial q_{i}}\right)=Q_{i}
$$

Onde $L$ é a Lagrangiana do sistema (diferença entre energia cinética e potencial), $q_{i}$ é a $i$-ésima coordenada generalizada e $Q_{i}$ são as forças generalizadas na direção da $i$-ésima coordenada.

As velocidades angulares $\omega_{x}, \omega_{y}$ e $\omega_{z}$ do pêndulo em torno do sistema de coordenadas $x y z$ não podem ser integradas para obtenção da posição e orientação do pêndulo no sistema de coordenadas $X Y Z$ e que, portanto, não são satisfatórias para aplicação das equações de Euler-Lagrange. Para descrever a orientação do pêndulo no espaço torna-se necessário a utilização de três coordenadas independentes. Esse conjunto de coordenadas não é necessariamente único, portanto, será utilizado um conjunto (bastante comum entre engenheiros, físicos e matemáticos) conhecido como ângulos de Euler. (Dion, 2008) (Dion and Nyln, 2013)

\section{Ângulos de Euler}

Os ângulos de Euler são um conjunto de três transformações lineares consecutivas, cada uma em torno de um eixo e com um deslocamento angular distinto, que podem levar um sistema de coordenadas fixo a qualquer outro sistema de coordenadas móvel. Além disso, as três componentes de velocidade angular do pêndulo podem ser expressas em termos dos ângulos de Euler e suas derivadas temporais. (Meirovitch, 2003)

Não há um acordo quanto à notação ultilizada pelos ângulos de Euler, porém uma bastante comum corresponde à seguinte sequência de rotações:

- Rotação de um ângulo $\phi$ em torno do eixo $Z$ (Figura 2.5a);

- Rotação de um ângulo $\theta$ em torno do eixo $y^{\prime \prime}$ (Figura 2.5b);

- Rotação de um ângulo $\psi$ em torno do eixo $z^{\prime}$ (Figura 2.5c).

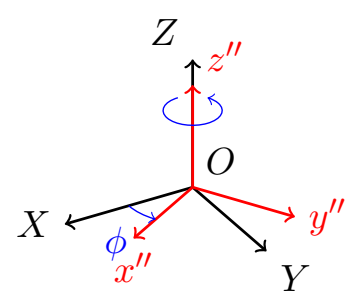

(a) Rotação de um ângulo $\phi$ em torno do eixo $Z$

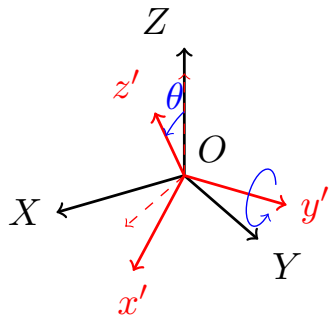

(b) Rotação de um ângulo $\theta$ em torno do eixo $y^{\prime \prime}$

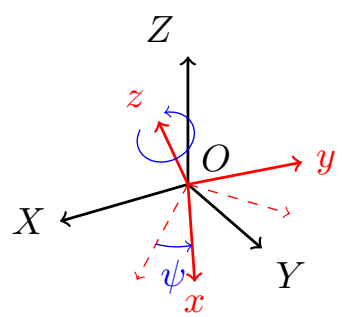

(c) Rotação de um ângulo $\psi$ em torno do eixo $z^{\prime}$

Figura 2.5: Notação utilizada nos ângulos de Euler 
Um problema na utilização dos ângulos de Euler está na ocorrências de singularidades. Singularidades são os pontos nos quais uma variável matemática torna-se indefinida, ou no caso dos ângulo de Euler, uma posição e orientação na qual há mais de uma única sequência de rotações possíveis. Por exemplo, na Figura 2.5, quando a rotação de um ângulo $\theta$ em torno do eixo $y^{\prime}$ é $n \pi \operatorname{com} n=0, \pm 1, \pm 2, \ldots$, os eixos da primeira $(Z)$ e terceira $\left(z^{\prime \prime}\right)$ rotação coincidem, assim torna-se impossível discernir os valores de $\phi$ e $\psi$.

Singularidades sempre vão ocorrer ao utilizar os ângulos de Euler, o que muda são as posições e orientações nas quais elas ocorrem. Como o objetivo é estabilizar o pêndulo em uma determinada posição e orientação de equilíbrio, deve ser adotada uma notação dos ângulos de Euler de maneira que as singularidades não ocorram próximas a esse ponto equilíbrio. Uma notação que satisfaz a essa condição corresponde à seguinte sequência de rotações:

- Rotação de um ângulo $\phi$ em torno do eixo $X$ (Figura 2.6a);

- Rotação de um ângulo $\theta$ em torno do eixo $y^{\prime \prime}$ (Figura 2.6b);

- Rotação de um ângulo $\psi$ em torno do eixo $z^{\prime}$ (Figura 2.6c);

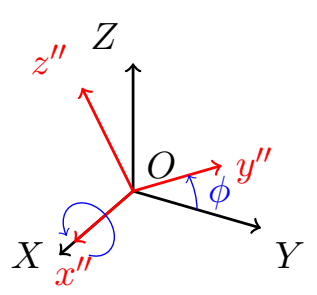

(a) Rotação de um ângulo $\phi$ em torno do eixo $X$

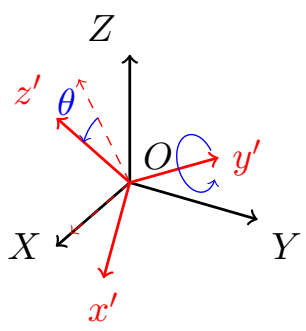

(b) Rotação de um ângulo $\theta$ em torno do eixo $y^{\prime \prime}$

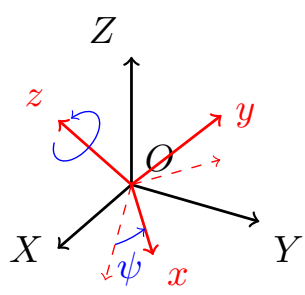

(c) Rotação de um ângulo $\psi$ em torno do eixo $z^{\prime}$

Figura 2.6: Notação utilizada nos ângulos de Bryant

Essa notação, com os três eixos de rotação distintos, é conhecida como ângulos de Tait-Bryant. Os ângulos de Tait-Bryant são um caso específico dos ângulos de Euler.

Nesse caso, as singularidades também ocorrem. Quando $\theta$ for igual a $\frac{\pi}{2}+n \pi$ com $n=0, \pm 1, \pm 2, \ldots, \phi$ e $\psi$ tornam-se indistinguíveis. Mas esses pontos estão muito longe da posição e orientação de equilíbrio do pêndulo. Portanto, essa notação é satisfatória para este estudo.

A matriz de rotação em torno do eixo $X$ por um ângulo $\phi$ é dada por:

$$
R_{x}(\phi)=\left[\begin{array}{ccc}
1 & 0 & 0 \\
0 & \cos \phi & -\operatorname{sen} \phi \\
0 & \operatorname{sen} \phi & \cos \phi
\end{array}\right]
$$

Onde: 


$$
\left[\begin{array}{l}
x^{\prime \prime} \\
y^{\prime \prime} \\
z^{\prime \prime}
\end{array}\right]=R_{x}(\phi)\left[\begin{array}{l}
X \\
Y \\
Z
\end{array}\right]
$$

A matriz de rotação em torno do eixo $y^{\prime \prime}$ por um ângulo $\theta$ é dada por:

$$
R_{y}(\theta)=\left[\begin{array}{ccc}
\cos \theta & 0 & \operatorname{sen} \theta \\
0 & 1 & 0 \\
-\operatorname{sen} \theta & 0 & \cos \theta
\end{array}\right]
$$

Onde:

$$
\left[\begin{array}{l}
x^{\prime} \\
y^{\prime} \\
z^{\prime}
\end{array}\right]=R_{y}(\theta)\left[\begin{array}{l}
x^{\prime \prime} \\
y^{\prime \prime} \\
z^{\prime \prime}
\end{array}\right]
$$

A matriz de rotação em torno do eixo $z^{\prime}$ por um ângulo $\psi$ é dada por:

$$
R_{z}(\psi)=\left[\begin{array}{ccc}
\cos \psi & -\operatorname{sen} \psi & 0 \\
\operatorname{sen} \psi & \cos \psi & 0 \\
0 & 0 & 1
\end{array}\right]
$$

Onde:

$$
\left[\begin{array}{l}
x \\
y \\
z
\end{array}\right]=R_{z}(\psi)\left[\begin{array}{l}
x^{\prime} \\
y^{\prime} \\
z^{\prime}
\end{array}\right]
$$

A transformação de coordenadas do sistema global para o sistema fixo no pêndulo pode então ser escrita na forma matricial da seguinte maneira:

$$
\left[\begin{array}{l}
x \\
y \\
z
\end{array}\right]=R\left[\begin{array}{l}
X \\
Y \\
Z
\end{array}\right]
$$

Onde $R$ é a matriz de rotação que representa o produto triplo de outras três matrizes de rotação:

$$
R=R_{z}(\psi) R_{y}(\theta) R_{x}(\phi)
$$

Assim, substituindo as Equações (2.2), (2.4) e (2.6) na Equação (2.9) tem-se ${ }^{1}$ :

$$
R=\left[\begin{array}{ccc}
c \theta c \psi & -c \phi s \psi+s \phi s \theta c \psi & s \phi s \psi+c \phi s \theta c \psi \\
c \theta s \psi & c \phi c \psi+s \phi s \theta s \psi & -s \phi c \psi+c \phi s \theta s \psi \\
-s \theta & s \phi c \theta & c \phi c \theta
\end{array}\right]
$$

\footnotetext{
${ }^{1}$ Para economizar espaço em alguns casos as abreviações $s=$ sen e $c=\cos$ foram utilizadas.
} 
Nota-se que $R$ representa uma transformação ortonormal entre dois sistemas cartesianos e, portanto, a sua matriz inversa é igual a sua matriz transposta. Dessa maneira, a transformação inversa da Equação (2.8) pode ser representada por:

$$
\left[\begin{array}{l}
X \\
Y \\
Z
\end{array}\right]=R^{T}\left[\begin{array}{l}
x \\
y \\
z
\end{array}\right]
$$

Se o pêndulo girar em torno dos eixos $x, y$ e $z$ com velocidades angulares $\omega_{x}, \omega_{y}$ e $\omega_{z}$, a matriz $R$ também vai variar no tempo. Essa variação da matriz de rotação no tempo, $\dot{R}$, com a velocidade angular do pêndulo é dada por:

$$
\dot{R}=\left[\begin{array}{ccc}
0 & -\omega_{z} & \omega_{y} \\
\omega_{z} & 0 & -\omega_{x} \\
-\omega_{y} & \omega_{x} & 0
\end{array}\right] R
$$

Ou, de maneira análoga:

$$
\left[\begin{array}{ccc}
0 & -\omega_{z} & \omega_{y} \\
\omega_{z} & 0 & -\omega_{x} \\
-\omega_{y} & \omega_{x} & 0
\end{array}\right]=\dot{R} R^{T}
$$

Substituindo a Equação (2.10) na Equação (2.13), tem-se:

$$
\left[\begin{array}{ccc}
0 & -\omega_{z} & \omega_{y} \\
\omega_{z} & 0 & -\omega_{x} \\
-\omega_{y} & \omega_{x} & 0
\end{array}\right]=\left[\begin{array}{ccc}
0 & -\dot{\psi}+\dot{\phi} s \theta & \dot{\theta} c \psi+\dot{\phi} c \theta s \psi \\
\dot{\psi}-\dot{\phi} s \theta & 0 & \dot{\theta} s \psi-\dot{\phi} c \theta c \psi \\
-\dot{\theta} c \psi-\dot{\phi} c \theta s \psi & -\dot{\theta} s \psi+\dot{\phi} c \theta c \psi & 0
\end{array}\right]
$$

Logo, da Equação (2.14) pode-se concluir que:

$$
\begin{aligned}
& \omega_{x}=-\dot{\theta} \operatorname{sen} \psi+\dot{\phi} \cos \theta \cos \psi \\
& \omega_{y}=\dot{\theta} \cos \psi+\dot{\phi} \cos \theta \operatorname{sen} \psi \\
& \omega_{z}=\dot{\psi}-\dot{\phi} \operatorname{sen} \theta
\end{aligned}
$$

Essas são as velocidades angulares do pêndulo em torno do sistema de coordenadas $x y z$ em função dos ângulos de Euler.

\section{Energia cinética}

A haste não apresenta nenhum movimento de translação, apenas de rotação. Dessa forma, a energia cinética do sistema pode ser escrita como:

$$
T=\frac{1}{2} J_{x} \omega_{x}^{2}+\frac{1}{2} J_{y} \omega_{y}^{2}+\frac{1}{2} J_{z} \omega_{z}^{2}
$$


Devido à simetria do pêndulo, o momento de inércia $J_{x}$ do pêndulo ao longo do eixo $x$ é igual ao momento de inércia $J_{y}$ ao longo do eixo $y$. Dessa forma, é possível simplificar a Equação (2.16):

$$
T=\frac{1}{2} J_{x}\left(\omega_{x}^{2}+\omega_{y}^{2}\right)+\frac{1}{2} J_{z} \omega_{z}^{2}
$$

Substituindo os valores das velocidades angulares $\omega_{x}, \omega_{y}$ e $\omega_{z}$ em termos dos ângulos de Euler obtidas nas Equações (2.15), pode-se reescrever a Equação (2.17) da seguinte maneira:

$$
T=\frac{1}{2} J_{x}\left(\dot{\theta}^{2}+\dot{\phi}^{2} \cos ^{2} \theta\right)+\frac{1}{2} J_{z}(\dot{\psi}-\dot{\phi} \operatorname{sen} \theta)^{2}
$$

\section{Energia potencial}

Apesar de não apresentar movimentos de translação, os movimentos de rotação dos ângulos $\phi$ e $\theta$ influenciam a posição vertical do centro de massa da haste. Logo, a energia potencial do sistema pode ser escrita como:

$$
V=m g z_{G} \cos \phi \cos \theta
$$

\section{Forças generalizadas}

Há três forças generalizadas no sub-sistema da haste, que são os três torques $\tau_{x}, \tau_{y}$ e $\tau_{z}$. Para aplicar as equações de Euler-Lagrange torna-se necessário decompor essas forças generalizadas na direção e sentido de cada uma das coordenadas generalizadas $\phi, \theta$ e $\psi$.

Com base na Figura 2.4, pode-se verificar que o torque $\tau_{x}$ ocorre na direção e sentido do eixo $-x, \tau_{y}$ na direção e sentido de $-y$ e $\tau_{z}$ de $-z$ :

$$
\left[\begin{array}{l}
Q_{x} \\
Q_{y} \\
Q_{z}
\end{array}\right]=\left[\begin{array}{l}
-\tau_{x} \\
-\tau_{y} \\
-\tau_{z}
\end{array}\right]
$$

Na Figura 2.6, verifica-se que a coordenada generalizada $\psi$ está na mesma direção e sentido do eixo $z, \theta$ na direção e sentido de $y^{\prime}$ e $\phi$ de $x^{\prime \prime}$, portanto as forças generalizadas são análogas:

$$
\left[\begin{array}{l}
Q_{\phi} \\
Q_{\theta} \\
Q_{\psi}
\end{array}\right]=\left[\begin{array}{l}
Q_{x^{\prime \prime}} \\
Q_{y^{\prime}} \\
Q_{z}
\end{array}\right]
$$

Logo, das Equações (2.21) e (2.20), pode-se concluir que na direção e sentido da coordenada generalizada $\psi$ ocorre a força generalizada $-\tau_{z}$.

A partir da Equação (2.6), tem-se que: 


$$
\left[\begin{array}{c}
Q_{x^{\prime}} \\
Q_{y^{\prime}} \\
Q_{z^{\prime}}
\end{array}\right]=R_{z}(\psi)^{T}\left[\begin{array}{l}
Q_{x} \\
Q_{y} \\
Q_{z}
\end{array}\right]
$$

Logo, das Equações (2.21) e (2.22), pode-se concluir que na direção e sentido da coordenada generalizada $\theta$ ocorre a força generalizada $\tau_{x} \operatorname{sen} \psi-\tau_{y} \cos \psi$.

A partir das Equações (2.4) e (2.6), tem-se que:

$$
\left[\begin{array}{l}
Q_{x^{\prime \prime}} \\
Q_{y^{\prime \prime}} \\
Q_{z^{\prime \prime}}
\end{array}\right]=\left(R_{z}(\psi) R_{y}(\theta)\right)^{T}\left[\begin{array}{l}
Q_{x} \\
Q_{y} \\
Q_{z}
\end{array}\right]
$$

Logo, das Equações (2.23) e (2.21), pode-se concluir que na direção e sentido da coordenada generalizada $\phi$ ocorre a força generalizada $-\tau_{x} \cos \theta \cos \psi-\tau_{y} \cos \theta \operatorname{sen} \psi+$ $\tau_{z} \operatorname{sen} \theta$.

Dessa forma, as forças generalizadas na direção e sentido das três coordenadas generalizadas são dadas por:

$$
\left\{\begin{array}{l}
Q_{\phi}=-\tau_{x} \cos \theta \cos \psi-\tau_{y} \cos \theta \operatorname{sen} \psi+\tau_{z} \operatorname{sen} \theta \\
Q_{\theta}=\tau_{x} \operatorname{sen} \psi-\tau_{y} \cos \psi \\
Q_{\psi}=-\tau_{z}
\end{array}\right.
$$

\section{Equações diferenciais}

A Lagrangiana é a diferença entre a energia cinética e a energia potencial do sistema. Dessa forma, das Equações (2.18) e (2.19) tem-se que:

$$
L=\frac{1}{2} J_{x}\left(\dot{\theta}^{2}+\dot{\phi}^{2} \cos ^{2} \theta\right)+\frac{1}{2} J_{z}(\dot{\psi}-\dot{\phi} \operatorname{sen} \theta)^{2}-m g z_{G} \cos \phi \cos \theta
$$

Como na Lagrangiana há três coordenadas generalizadas, três equações diferenciais deverão ser obtidas para o sub-sistema da haste:

Para $q_{i}=\phi$ tem-se:

$$
\begin{aligned}
\frac{d}{d t}\left(\frac{\partial L}{\partial \dot{\phi}}\right)= & \frac{d}{d t}\left(J_{x} \dot{\phi} \cos ^{2} \theta-J_{z}(\dot{\psi}-\dot{\phi} \operatorname{sen} \theta) \operatorname{sen} \theta\right) \\
= & J_{x}\left(\ddot{\phi} \cos ^{2} \theta-\dot{\phi} \dot{\theta} \operatorname{sen}(2 \theta)\right) \\
& \quad+J_{z}\left(\ddot{\phi} \operatorname{sen}^{2} \theta-\ddot{\psi} \operatorname{sen} \theta-\dot{\theta} \dot{\psi} \cos \theta+\dot{\phi} \dot{\theta} \operatorname{sen}(2 \theta)\right) \\
\left(\frac{\partial L}{\partial \phi}\right)= & m g z_{G} \operatorname{sen} \phi \cos \theta \\
Q_{\phi}= & -\tau_{x} \cos \theta \cos \psi-\tau_{y} \cos \theta \operatorname{sen} \psi+\tau_{z} \operatorname{sen} \theta
\end{aligned}
$$


Para $q_{i}=\theta$ tem-se:

$$
\begin{aligned}
\frac{d}{d t}\left(\frac{\partial L}{\partial \dot{\theta}}\right) & =\frac{d}{d t}\left(J_{x} \dot{\theta}\right) \\
& =J_{x} \ddot{\theta} \\
\left(\frac{\partial L}{\partial \theta}\right) & =-J_{x} \dot{\phi}^{2} \operatorname{sen} \theta \cos \theta+J_{z}(\dot{\phi} \operatorname{sen} \theta-\dot{\psi}) \dot{\phi} \cos \theta+m g z_{G} \cos \phi \operatorname{sen} \theta \\
Q_{\theta} & =\tau_{x} \operatorname{sen} \psi-\tau_{y} \cos \psi
\end{aligned}
$$

Para $q_{i}=\psi$ tem-se:

$$
\begin{aligned}
\frac{d}{d t}\left(\frac{\partial L}{\partial \dot{\psi}}\right) & =\frac{d}{d t}\left(J_{z}(\dot{\psi}-\dot{\phi} \operatorname{sen} \theta)\right) \\
& =J_{z}(\ddot{\psi}-\ddot{\phi} \operatorname{sen} \theta-\dot{\phi} \dot{\theta} \cos \theta) \\
\left(\frac{\partial L}{\partial \psi}\right) & =0 \\
Q_{\psi} & =-\tau_{z}
\end{aligned}
$$

Portanto, da Equação (2.1) são obtidas as seguintes equações diferenciais do subsistema da haste:

$$
\left\{\begin{array}{c}
J_{x}\left(\ddot{\phi} \cos ^{2} \theta-\dot{\phi} \dot{\theta} \operatorname{sen}(2 \theta)\right)+J_{z}\left(\ddot{\phi} \operatorname{sen}^{2} \theta-\ddot{\psi} \operatorname{sen} \theta-\dot{\theta} \dot{\psi} \cos \theta+\dot{\phi} \dot{\theta} \operatorname{sen}(2 \theta)\right) \\
-m g z_{G} \operatorname{sen} \phi \cos \theta=-\tau_{x} \cos \theta \cos \psi-\tau_{y} \cos \theta \operatorname{sen} \psi+\tau_{z} \operatorname{sen} \theta \\
J_{x}\left(\ddot{\theta}+\dot{\phi}^{2} \operatorname{sen} \theta \cos \theta\right)+J_{z}(\dot{\psi}-\dot{\phi} \operatorname{sen} \theta) \dot{\phi} \cos \theta-m g z_{G} \cos \phi \operatorname{sen} \theta=\tau_{x} \operatorname{sen} \psi-\tau_{y} \cos \psi \\
J_{z}(\ddot{\psi}-\ddot{\phi} \operatorname{sen} \theta-\dot{\phi} \dot{\theta} \cos \theta)=-\tau_{z}
\end{array}\right.
$$

\subsection{Modelagem das rodas de reação}

O sub-sistema das rodas de reação possui como entrada os torques desenvolvidos pelos motores elétricos $\bar{\tau}_{x}, \bar{\tau}_{y}$ e $\bar{\tau}_{z}$ e, como saída, os torques úteis de reação desenvolvidos pelas rodas de reação $\tau_{x}, \tau_{y}$ e $\tau_{z}$ e as velocidades angulares das rodas de reação $\dot{\alpha}, \dot{\beta}$ e $\dot{\gamma}$, conforme a Figura 2.7.

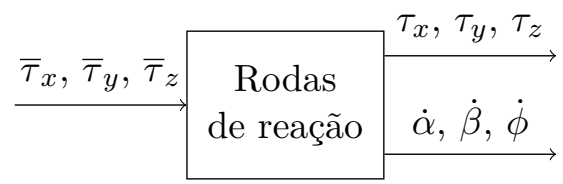

Figura 2.7: Diagrama de blocos do sub-sistema das rodas de reação 
Como todas as três rodas de reação são iguais, apenas a roda de reação do eixo $x$ será modelada e o modelo obtido será replicado às demais. A dinâmica da roda de reação pode ser observada na Figura 2.8 e seus parâmetros estão definidos na Tabela 2.2 .

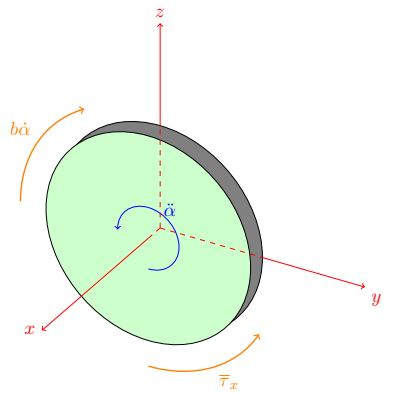

Figura 2.8: Dinâmica do sub-sistema das rodas de reação

Tabela 2.2: Parâmetros do sub-sistema da roda de reação

\begin{tabular}{c|l} 
Símbolo & Parâmetro \\
\hline $\bar{\tau}_{x}$ & Torque desenvolvido pelo motor \\
$\tau_{x}$ & Torque útil de reação desenvolvido pela roda de reação \\
$J_{r}$ & Momento de inércia das rodas de reação em torno de seu eixo longitudinal \\
$b$ & Coeficiente de atritos viscosos da roda de reação \\
$\ddot{\alpha}$ & Aceleração angular da roda de reação
\end{tabular}

\subsubsection{Modelagem pelas leis de Newton}

A segunda lei de Newton diz que: "A atuação de forças externas atuantes em um corpo provoca aceleração. Tal aceleração possui a mesma direção e o mesmo sentido da força resultante que atua no corpo. Além disso, o vetor força resultante é numericamente igual ao produto da massa do corpo pelo respectivo vetor aceleração.". Algebricamente, isto significa que:

$$
F=m \ddot{x}
$$

Para sistemas mecânicos de rotação, a lei é análoga aos sistemas de translação, ou seja:

$$
\tau=J \ddot{\alpha}
$$

Aplicando essa lei no eixo da roda de reação tem-se que:

$$
\bar{\tau}_{x}-b \dot{\alpha}=J_{r} \ddot{\alpha}
$$

Rearranjando a equação: 


$$
\ddot{\alpha}=-\frac{b}{J_{r}} \dot{\alpha}+\frac{1}{J_{r}} \bar{\tau}_{x}
$$

Portanto, as equações diferenciais do sub-sistema das rodas de reação são dadas por:

$$
\left\{\begin{array}{l}
\ddot{\alpha}=-\frac{b}{J_{r}} \dot{\alpha}+\frac{1}{J_{r}} \bar{\tau}_{x} \\
\ddot{\beta}=-\frac{b}{J_{r}} \dot{\beta}+\frac{1}{J_{r}} \bar{\tau}_{y} \\
\ddot{\gamma}=-\frac{b}{J_{r}} \dot{\gamma}+\frac{1}{J_{r}} \bar{\tau}_{z}
\end{array}\right.
$$

\subsection{Modelagem dos motores elétricos}

O sub-sistema dos motores elétricos possui como entrada as tensões aplicadas em cada um dos motores $e_{x}, e_{y}$ e $e_{z}$ e as velocidades angulares das rodas de reação $\dot{\alpha}, \dot{\beta}$ e $\dot{\gamma}$ e, como saída, os torques desenvolvidos pelos motores elétricos $\tau_{x}, \tau_{y}$ e $\tau_{z}$, conforme a Figura 2.9.

$\stackrel{e_{x}, e_{y}, e_{z}}{\stackrel{\alpha}{\beta}, \dot{\beta}, \dot{\gamma}} \underset{\begin{array}{l}\text { Motores } \\ \text { elétricos }\end{array}}{\stackrel{\bar{\tau}_{x}, \bar{\tau}_{y}, \bar{\tau}_{z}}{\longrightarrow}}$

Figura 2.9: Diagrama de blocos do sub-sistema dos motores elétricos

Como os três motores elétricos são iguais, apenas o motor elétrico do eixo $x$ será modelado e o modelo obtido será replicado aos demais. A dinâmica de um motor de corrente contínua pode ser observada na Figura 2.10 e seus parâmetros estão definidos na Tabela 2.3.

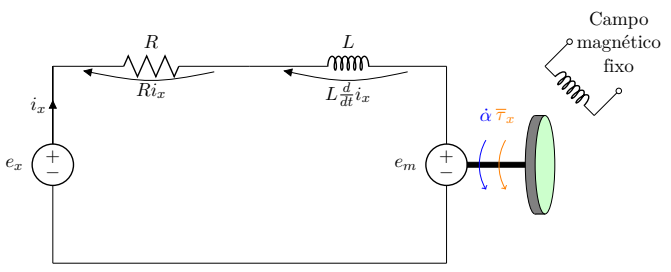

Figura 2.10: Dinâmica do sub-sistema dos motores elétricos 
Tabela 2.3: Parâmetros do sub-sistema do motor elétrico

\begin{tabular}{c|l} 
Símbolo & Parâmetro \\
\hline$e_{x}$ & Tensão de armadura \\
$e_{m}$ & Tensão induzida (força contra-eletromotriz) \\
$i_{x}$ & Corrente de armadura \\
$R$ & Resistência do circuito de armadura \\
$L$ & Indutância do circuito de armadura \\
$k_{m}$ & Constante de densidade do fluxo \\
$k_{\tau}$ & Constante de torque \\
$\dot{\alpha}$ & Velocidade angular do motor \\
$\bar{\tau}_{x}$ & Torque desenvolvido pelo motor
\end{tabular}

A tensão aplicada no motor $e_{x}$ é conhecida como tensão de armadura. A resistência e a indutância do circuito de armadura são indicadas por $R$ e $L$. Quando a armadura está em rotação, uma tensão proporcional ao produto da densidade de fluxo pela velocidade angular $\dot{\alpha}$ do motor é induzida nela. No caso do motor de corrente contínua, o campo magnético é fixo e, assim, a densidade de fluxo magnético é constante. Dessa forma, a tensão induzida $e_{m}$, também chamada de força contra-eletromotriz, é diretamente proporcional a velocidade angular $\dot{\alpha}$. O torque $\bar{\tau}_{x}$ desenvolvido pelo motor é diretamente proporcional a corrente de armadura $i_{x}$.

\subsubsection{Modelagem pelas leis de Kirchhoff}

A segunda lei de Kirchhoff diz que "A soma algébrica das tensões em qualquer malha de um circuito elétrico é nula.". Algebricamente ela diz que:

$$
\sum_{k=1}^{n} e_{k}=0
$$

Aplicando essa lei de na malha do circuito de armadura tem-se que:

$$
e_{x}-L \frac{d}{d t} i_{x}-R i_{x}-e_{m}=0
$$

Sabendo que a tensão induzida $e_{m}$ é diretamente proporcional a velocidade angular $\dot{\alpha}$ do motor e que o torque $\bar{\tau}_{x}$ desenvolvido pelo motor é diretamente proporcional a corrente de armadura $i_{x}$, tem-se que:

$$
e_{m}=k_{m} \dot{\alpha}, \quad \bar{\tau}_{x}=k_{\tau} i_{x}
$$

Substituindo na Equação (2.36):

$$
e_{x}-\frac{L}{k_{\tau}} \dot{\bar{\tau}_{x}}-\frac{R}{k_{\tau}} \bar{\tau}_{x}-k_{m} \dot{\alpha}=0
$$

Rearranjando a equação: 


$$
\dot{\bar{\tau}}_{x}=-\frac{R}{L} \bar{\tau}_{x}-\frac{k_{m} k_{\tau}}{L} \dot{\alpha}+\frac{k_{\tau}}{L} e_{x}
$$

Portanto, as equações diferenciais do sub-sistema dos motores elétricos são dadas por:

$$
\left\{\begin{array}{c}
\dot{\bar{\tau}}_{x}=-\frac{R}{L} \bar{\tau}_{x}-\frac{k_{m} k_{\tau}}{L} \dot{\alpha}+\frac{k_{\tau}}{L} e_{x} \\
\dot{\bar{\tau}}_{y}=-\frac{R}{L} \bar{\tau}_{y}-\frac{k_{m} k_{\tau}}{L} \dot{\beta}+\frac{k_{\tau}}{L} e_{y} \\
\dot{\bar{\tau}_{z}}=-\frac{R}{L} \bar{\tau}_{z}-\frac{k_{m} k_{\tau}}{L} \dot{\gamma}+\frac{k_{\tau}}{L} e_{z}
\end{array}\right.
$$

\subsection{Definição dos parâmetros}

Para a realização de simulações, todos os parâmetros do pêndulo precisam ter valores numéricos. Os parâmetros podem ser determinados a partir de um modelo tridimensional do sistema ou por experiências diretas em um modelo físico. É muito útil combinar ambos os métodos e fazer verificações cruzadas. Como nesse trabalho não foi construído um modelo físico do sistema, os parâmetros serão determinados apenas a partir de um modelo tridimensional e algumas premissas serão adotadas.

O massa do pêndulo é composta basicamente por seis elementos ${ }^{2}$ : uma haste, três rodas de reação e dois contra-pesos. A haste pode ser aproximada a um cilindro sólido, enquanto que as rodas de reação e os contra-pesos podem ser aproximados a cilindros $\operatorname{ocos}^{3}$, conforme a Figura 2.11.

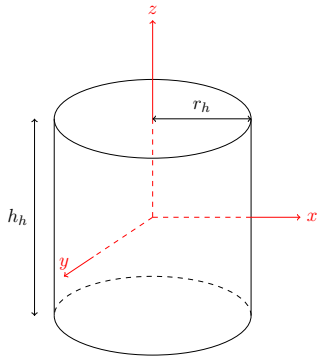

(a) Cilindro sólido

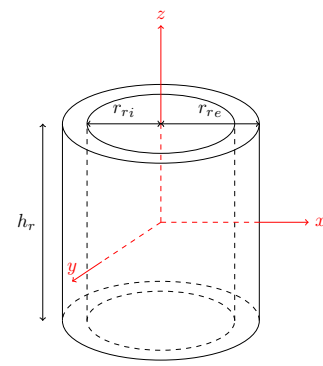

(b) Cilindro oco

Figura 2.11: Dimensões de cilindros

Para a haste, foram adotadas as seguintes dimensões:

$$
\left\{\begin{array}{l}
h_{h}=20 \mathrm{~cm} \\
r_{h}=0,5 \mathrm{~cm}
\end{array}\right.
$$

\footnotetext{
${ }^{2}$ Assumindo que a massa dos motores elétricos possa ser desprezada ou já esteja considerada na massa do pêndulo

${ }^{3}$ As rodas de reação foram aproximadas a cilindros ocos e não sólidos pois dessa forma sua massa fica concentrada na extremidade, aumentando assim seu momento de inércia sem aumentar muito sua massa total
} 
Para as rodas de reação e contra-pesos foram adotadas as seguintes dimensões:

$$
\left\{\begin{array}{l}
h_{r}=1 \mathrm{~cm} \\
r_{r i}=2,0 \mathrm{~cm} \\
r_{r e}=2,5 \mathrm{~cm}
\end{array}\right.
$$

A haste deve ser fabricada de um material leve, como por exemplo um alumínio cuja densidade é:

$$
\rho_{h}=2,7 \mathrm{~g} / \mathrm{cm}^{3}
$$

Dessa forma, a massa da haste é dada por:

$$
\begin{aligned}
m_{h} & =\rho_{h} V_{h} \\
m_{h} & =\rho_{h}\left(\pi r_{h}{ }^{2} h_{h}\right) \\
m_{h} & =0,42 g
\end{aligned}
$$

Já as rodas de reação e os contra-pesos devem ser fabricados de um material mais pesado, como por exemplo um aço cuja densidade é:

$$
\rho_{r}=7,86 \mathrm{~g} / \mathrm{cm}^{3}
$$

Dessa forma, a massa das rodas de reação e dos contra-pesos é dada por:

$$
\begin{aligned}
& m_{r}=\rho_{r} V_{r} \\
& m_{r}=\rho_{r}\left(\pi\left(r_{r e}-r_{r i}\right)^{2} h_{r}\right) \\
& m_{r}=55,60 \mathrm{~g}
\end{aligned}
$$

Dado que o pêndulo é composto pela haste, três rodas de reação e dois contrapesos (cujas dimensões são idênticas as das rodas de reação), sua massa é dada por:

$$
\begin{aligned}
& m=m_{h}+3 m_{r}+2 m_{r} \\
& m=278,2 g
\end{aligned}
$$

A distância da articulação esférica até o centro de massa da haste é $h_{h} / 2$. Já a distância da articulação esférica até o centro de massa das três rodas de reação e dois contrapesos é igual a $h_{h}$. Dessa forma, a distância da articulação esférica até o centro de massa do pêndulo $\left(z_{G}\right)$ é dada por:

$$
\begin{aligned}
z_{G} & =\frac{m_{h} h_{h} / 2+3 m_{r} h_{h}+2 m_{r} h_{h}}{m} \\
z_{G} & =19,98 \mathrm{~cm}
\end{aligned}
$$


Os momentos de inércia de um cilindro sólido de massa $m_{h}$, como o da Figura 2.11a, são dados por:

$$
\left\{\begin{array}{l}
J_{h x}=\frac{1}{12} m_{h}\left(3 r_{h}{ }^{2}+h_{h}{ }^{2}\right) \\
J_{h y}=\frac{1}{12} m_{h}\left(3 r_{h}{ }^{2}+h_{h}{ }^{2}\right) \\
J_{h z}=\frac{1}{2} m_{h} r_{h}{ }^{2}
\end{array}\right.
$$

Dessa forma, os momentos de inércia da haste são:

$$
\left\{\begin{array}{l}
J_{h x}=1,41 \times 10^{-6} \mathrm{~kg} \cdot \mathrm{m}^{2} \\
J_{h y}=1,41 \times 10^{-6} \mathrm{~kg} \cdot \mathrm{m}^{2} \\
J_{h z}=5,30 \times 10^{-11} \mathrm{~kg} \cdot \mathrm{m}^{2}
\end{array}\right.
$$

Os momentos de inércia de um cilindro oco de massa $m_{r}$, como o da Figura 2.11b, são dados por:

$$
\left\{\begin{array}{l}
J_{r x}=\frac{1}{12} m_{r}\left(3\left(r_{r e}{ }^{2}+r_{r i}{ }^{2}\right)+{h_{r}}^{2}\right) \\
J_{r y}=\frac{1}{12} m_{r}\left(3\left(r_{r e}{ }^{2}+r_{r i}{ }^{2}\right)+{h_{r}}^{2}\right) \\
J_{r z}=\frac{1}{2} m_{r}\left(r_{r e}{ }^{2}+r_{r i}{ }^{2}\right)
\end{array}\right.
$$

Dessa forma, os momentos de inércia das rodas de reação e contra-pesos são:

$$
\left\{\begin{array}{l}
J_{r x}=1,47 \times 10^{-5} \mathrm{~kg} \cdot \mathrm{m}^{2} \\
J_{r y}=1,47 \times 10^{-5} \mathrm{~kg} \cdot \mathrm{m}^{2} \\
J_{r z}=2,85 \times 10^{-5} \mathrm{~kg} \cdot \mathrm{m}^{2}
\end{array}\right.
$$

Assim, os momentos de inércia do pêndulo em torno de sua articulação esférica são dados por:

$$
\begin{aligned}
& J_{x}=\left(J_{h x}+m_{h}\left(\frac{h_{h}}{2}\right)^{2}\right)+2\left(J_{r z}+m_{r} h_{h}^{2}\right)+2\left(J_{r x}+m_{r} h_{h}^{2}\right)+\left(J_{r x}+m_{r}\left(h_{h}+e\right)^{2}\right) \\
& J_{x}=1,25 \times 10^{-2} \mathrm{~kg} \cdot \mathrm{m}^{2} \\
& J_{y}=\left(J_{h y}+m_{h}\left(\frac{h_{h}}{2}\right)^{2}\right)+2\left(J_{r z}+m_{r} h_{h}^{2}\right)+2\left(J_{r y}+m_{r} h_{h}{ }^{2}\right)+\left(J_{r y}+m_{r}\left(h_{h}+e\right)^{2}\right) \\
& J_{y}=1,25 \times 10^{-2} \mathrm{~kg} \cdot \mathrm{m}^{2} \\
& J_{z}=\left(J_{h z}\right)+\left(J_{r z}\right)+4\left(J_{r x}+m_{r} e^{2}\right)
\end{aligned}
$$


O momento de inércia das rodas de reação em torno de seus eixos longitudinais é dado por:

$$
\begin{aligned}
& J_{r}=J_{r z} \\
& J_{r}=2,85 \times 10^{-5} \mathrm{~kg} \cdot \mathrm{m}^{2}
\end{aligned}
$$

O valor do coeficiente de atritos viscosos das rodas de reação foi assumido como sendo muito pequeno:

$$
b=5 \times 10^{-3} \mathrm{~kg} \cdot \mathrm{m}^{2} / \mathrm{s}
$$

Os valores da resistência e indutância de armadura e as constantes de densidade de fluxo e torque dos motores foram adotados a partir de especificações de motores de corrente contínua típicos:

$$
\begin{gathered}
R=1 \Omega, \quad L=6 \times 10^{-3} \mathrm{H} \\
k_{m}=1 \times 10^{-3} \mathrm{~N} \cdot \mathrm{m} / \mathrm{A}, \quad k_{\tau}=1 \times 5^{-2} \mathrm{~V} /(\mathrm{rad} \cdot \mathrm{s})
\end{gathered}
$$

Dessa forma, temos os valores dos parâmetros do sistema no S.I. podem ser observados na Tabela 2.4 .

Tabela 2.4: Parâmetros adotados para o sistema

\begin{tabular}{c|c} 
Parâmetro & Valor adotado \\
\hline$m$ & $0,2782 \mathrm{~kg}$ \\
$z_{G}$ & $0,0182 \mathrm{~m}$ \\
$J_{x}$ & $1,25 \times 10^{-2} \mathrm{~kg} \cdot \mathrm{m}^{2}$ \\
$J_{y}$ & $1,25 \times 10^{-2} \mathrm{~kg} \cdot \mathrm{m}^{2}$ \\
$J_{z}$ & $6,42 \times 10^{-4} \mathrm{~kg} \cdot \mathrm{m}^{2}$ \\
$J_{r}$ & $2,85 \times 10^{-5} \mathrm{~kg} \cdot \mathrm{m}^{2}$ \\
$b$ & $5 \times 10^{-3} \mathrm{~kg} \cdot \mathrm{m}^{2} / \mathrm{s}$ \\
$R$ & $12 \Omega$ \\
$L$ & $6 \times 10^{-3} \mathrm{H}$ \\
$k_{m}$ & $1 \times 10^{-3} \mathrm{~V} /(\mathrm{rad} \cdot \mathrm{s})$ \\
$k_{\tau}$ & $1 \times 5^{-2} \mathrm{~N} \cdot \mathrm{m} / \mathrm{A}$ \\
$g$ & $9,81 \mathrm{~m} / \mathrm{s}^{2}$
\end{tabular}





\section{Capítulo 3}

\section{Análise}

Uma vez definido o modelo matemático do sistema a ser controlado, será feita a análise do mesmo. Neste capítulo, o sistema e seus respectivos sub-sistemas são analisados na mesma ordem em que foram modelados: primeiro a haste, depois a haste com as rodas de reação e, por fim, a haste com as rodas de reação e os motores elétricos.

\subsection{Definição da sequência de análise}

Conforme foi visto na Seção 2.1, o sistema completo do pêndulo invertido é composto por três sub-sistemas:

- Motores elétricos

- Rodas de reação

- Haste

Ele pode ser representado conforme o diagrama de blocos da Figura 3.1.

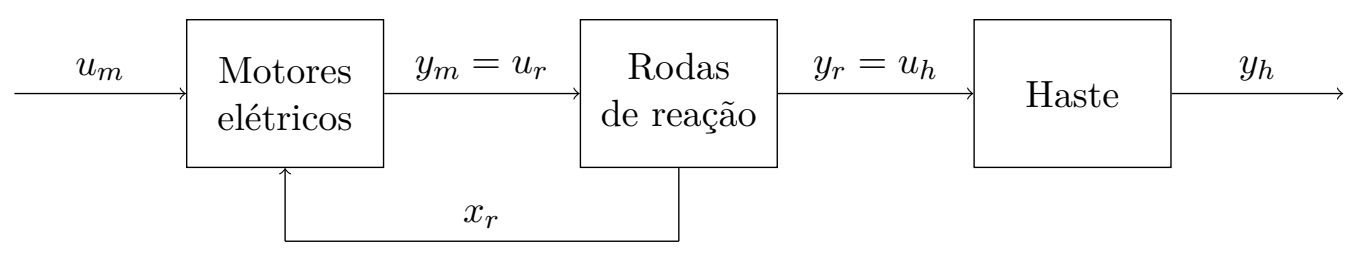

Figura 3.1: Diagrama de blocos do sistema completo

Onde:

$$
u_{m}=\left[\begin{array}{c}
e_{x} \\
e_{y} \\
e_{z}
\end{array}\right], \quad y_{m}=u_{r}=\left[\begin{array}{c}
\bar{\tau}_{x} \\
\bar{\tau}_{y} \\
\bar{\tau}_{z}
\end{array}\right], \quad y_{r}=u_{h}=\left[\begin{array}{c}
\tau_{x} \\
\tau_{y} \\
\tau_{z}
\end{array}\right], \quad y_{h}=\left[\begin{array}{c}
\phi \\
\theta \\
\psi
\end{array}\right]
$$

Para facilitar o entendimento, inicialmente o sistema considerado será composto apenas pelo sub-sistema da haste. Em seguida, o sistema considerado será composto pelo sub-sistema da haste e pelo sub-sistema das rodas de reação. Por fim, o sistema 
considerado será o completo, composto pelos três sub-sistemas: haste, rodas de reação e motores elétricos.

\subsection{Análise da haste}

Inicialmente, o sistema considerado será composto apenas pela haste, conforme o diagrama de blocos da Figura 3.2:

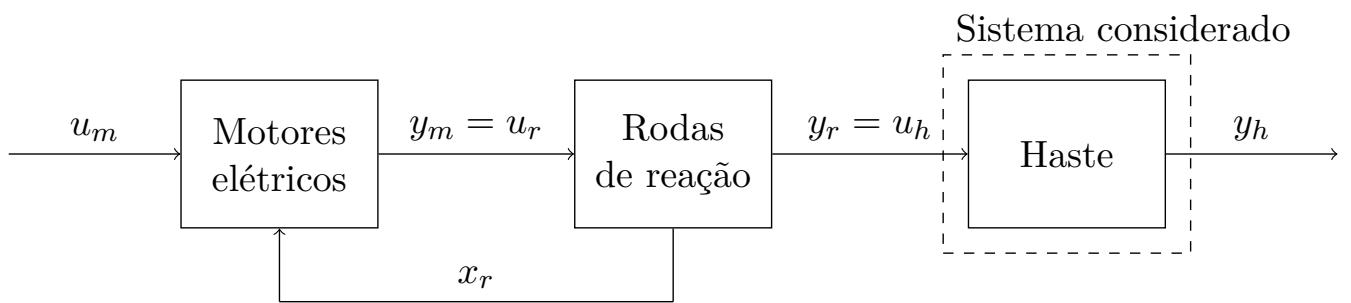

Figura 3.2: Diagrama de blocos do sistema completo e considerado (haste)

Dessa forma, o único objetivo é definir o sub-sistema da haste.

\subsubsection{Sub-sistema da haste}

Conforme deduzido na Seção 2.2, as equações diferenciais do sub-sistema da haste são dadas por:

$$
\left\{\begin{array}{c}
J_{x}\left(\ddot{\phi} \cos ^{2} \theta-\dot{\phi} \dot{\theta} \operatorname{sen}(2 \theta)\right)+J_{z}\left(\ddot{\phi} \operatorname{sen}^{2} \theta-\ddot{\psi} \operatorname{sen} \theta-\dot{\theta} \dot{\psi} \cos \theta+\dot{\phi} \dot{\theta} \operatorname{sen}(2 \theta)\right) \\
\quad-m g z_{G} \operatorname{sen} \phi \cos \theta=-\tau_{x} \cos \theta \cos \psi-\tau_{y} \cos \theta \operatorname{sen} \psi+\tau_{z} \operatorname{sen} \theta \\
J_{x}\left(\ddot{\theta}+\dot{\phi}^{2} \operatorname{sen} \theta \cos \theta\right)+J_{z}(\dot{\psi}-\dot{\phi} \operatorname{sen} \theta) \dot{\phi} \cos \theta-m g z_{G} \cos \phi \operatorname{sen} \theta=\tau_{x} \operatorname{sen} \psi-\tau_{y} \cos \psi \\
J_{z}(\ddot{\psi}-\ddot{\phi} \operatorname{sen} \theta-\dot{\phi} \dot{\theta} \cos \theta)=-\tau_{z}
\end{array}\right.
$$

Nota-se que esse sub-sistema é um sistema não-linear de 3 equações diferenciais ordinárias de $2^{\mathrm{a}}$ ordem.

\section{Rearranjo das equações do sub-sistema}

A haste trata-se de um sistema puramente mecânico, o que permite também rescrevê-lo da seguinte forma:

$$
M_{h}\left(q_{h}\right) \ddot{q}_{h}+N_{h}\left(q_{h}, \dot{q}_{h}\right)+O_{h}\left(q_{h}\right)=P_{h}\left(q_{h}\right) u_{h}
$$

Onde:

- $M_{h}$ é a matriz de massa do sub-sistema da haste

- $N_{h}$ é o vetor dos efeitos de Coriolis e atritos viscosos do sub-sistema da haste

- $O_{h}$ é o vetor dos efeitos de mola e de gravidade do sub-sistema da haste 
- $P_{h}$ é o a matriz de acoplamento das entradas do sub-sistema da haste

- $q_{h}$ é a vetor de coordenadas generalizadas do sub-sistema da haste

- $u_{h}$ é o vetor de entradas do sub-sistema da haste

Uma maneira de analisar um sistema de equações diferenciais ordinárias de segunda ordem é reescrevendo as equações de maneira que as segundas derivadas dependam apenas das primeiras derivadas e das próprias funções. Isso significa reescrever a Equação (3.3) da seguinte forma:

$$
\ddot{q}_{h}=-M_{h}^{-1}\left[N_{h}+O_{h}\right]+M_{h}^{-1} P_{h} u_{h}
$$

As coordenadas generalizadas desse sub-sistema são os ângulos de Euler $\phi, \theta$ e $\psi$ e as entradas são os toques úteis $\tau_{x}, \tau_{y}$ e $\tau_{z}$ :

$$
q_{h}=\left[\begin{array}{c}
\phi \\
\theta \\
\psi
\end{array}\right], \quad u_{h}=\left[\begin{array}{c}
\tau_{x} \\
\tau_{y} \\
\tau_{z}
\end{array}\right]
$$

Assim, $M_{h} \ddot{q}_{h}, N_{h}, O_{h}$ e $P_{h} u_{h}$ são obtidas com base nas Equações (3.2):

$$
\begin{gathered}
M_{h} \ddot{q}_{h}=\left[\begin{array}{c}
J_{x} \ddot{\phi} \cos ^{2} \theta+J_{z}\left(\ddot{\phi} \operatorname{sen}^{2} \theta-\ddot{\psi} \operatorname{sen} \theta\right) \\
J_{x} \ddot{\theta} \\
J_{z}(\ddot{\psi}-\ddot{\phi} \operatorname{sen} \theta)
\end{array}\right] \\
N_{h}=\left[\begin{array}{c}
-J_{x} \dot{\phi} \dot{\theta} \operatorname{sen}(2 \theta)-J_{z}(\dot{\theta} \dot{\psi} \cos \theta+\dot{\phi} \dot{\theta} \operatorname{sen}(2 \theta)) \\
J_{x} \dot{\phi}^{2} \operatorname{sen} \theta \cos \theta+J_{z}(\dot{\psi}-\dot{\phi} \operatorname{sen} \theta) \dot{\phi} \cos \theta \\
-J_{z} \dot{\phi} \dot{\theta} \cos \theta
\end{array}\right] \\
P_{h} u_{h}=\left[\begin{array}{c}
-m g z_{G} \operatorname{sen} \phi \cos \theta \\
-m g z_{G} \cos \phi \operatorname{sen} \theta \\
0
\end{array}\right] \\
O_{h}=\left[\begin{array}{c}
-\tau_{x} \cos \theta \cos \psi-\tau_{y} \cos \theta \operatorname{sen} \psi+\tau_{z} \operatorname{sen} \theta \\
\tau_{x} \operatorname{sen} \psi-\tau_{y} \cos \psi \\
-\tau_{z}
\end{array}\right]
\end{gathered}
$$

Já $M_{h}$ e $P_{h}$ são calculadas respectivamentes através dos Jacobianos de $M_{h} \ddot{q}_{h}$ com relação a $\ddot{q}_{h}$ e $P_{h} u_{h}$ com relação a $u_{h}$ :

$$
M_{h}=\left[\begin{array}{ccc}
\frac{\partial M_{h} \ddot{q}_{h_{1}}}{\partial \ddot{q}_{h_{1}}} & \ldots & \frac{\partial M_{h} \ddot{q}_{h_{1}}}{\partial \ddot{q}_{h_{n}}} \\
\vdots & \ddots & \vdots \\
\frac{\partial M_{h} \ddot{q}_{h_{n}}}{\partial \ddot{q}_{h_{1}}} & \ldots & \frac{\partial M_{h} \ddot{q}_{h_{n}}}{\partial \ddot{q}_{h_{n}}}
\end{array}\right], \quad P_{h}=\left[\begin{array}{ccc}
\frac{\partial P_{h} u_{h_{1}}}{\partial u_{h_{1}}} & \ldots & \frac{\partial P_{h} u_{h_{1}}}{\partial u_{h_{n}}} \\
\vdots & \ddots & \vdots \\
\frac{\partial P_{h} u_{h_{n}}}{\partial u_{h_{1}}} & \ldots & \frac{\partial P_{h} u_{h_{n}}}{\partial u_{h_{n}}}
\end{array}\right]
$$

Logo: 


$$
\begin{gathered}
M_{h}=\left[\begin{array}{ccc}
J_{x} \cos ^{2} \theta+J_{z} \operatorname{sen}^{2} \theta & 0 & -J_{z} \operatorname{sen} \theta \\
0 & J_{x} & 0 \\
-J_{z} \operatorname{sen} \theta & 0 & J_{z}
\end{array}\right] \\
P_{h}=\left[\begin{array}{ccc}
-\cos \theta \cos \psi & -\cos \theta \operatorname{sen} \psi & \operatorname{sen} \theta \\
\operatorname{sen} \psi & -\cos \psi & 0 \\
0 & 0 & -1
\end{array}\right]
\end{gathered}
$$

Substituindo essas matrizes na Equação (3.4), tem-se o mesmo sistema de equações diferenciais ordinárias, porém com as segundas derivadas dependendo apenas das primeiras derivadas e das próprias funções:

$$
\left\{\begin{aligned}
\ddot{\phi} & =2 \frac{\dot{\phi} \dot{\theta} \operatorname{sen} \theta}{\cos \theta}+\frac{J_{z}}{J_{x}} \frac{\dot{\theta}(\dot{\psi}-\dot{\phi} \operatorname{sen} \theta)}{\cos \theta}+\frac{m g z_{G}}{J_{x}} \frac{\operatorname{sen} \phi}{\cos \theta}-\frac{1}{J_{x}} \frac{\tau_{x} \cos \psi+\tau_{y} \operatorname{sen} \psi}{\cos \theta} \\
\ddot{\theta} & =-\frac{1}{2} \dot{\phi}^{2} \operatorname{sen}(2 \theta)-\frac{J_{z}}{J_{x}} \dot{\phi} \cos \theta(\dot{\psi}-\dot{\phi} \operatorname{sen} \theta)+\frac{m g z_{G}}{J_{x}} \cos \phi \operatorname{sen} \theta+\frac{1}{J_{x}}\left(\tau_{x} \operatorname{sen} \psi-\tau_{y} \cos \psi\right) \\
\ddot{\psi} & =-\frac{\dot{\phi} \theta\left(\cos ^{2} \theta-2\right)}{\cos \theta}+\frac{J_{z}}{J_{x}} \frac{\dot{\theta} \operatorname{sen} \theta(\dot{\psi}-\phi \operatorname{sen} \theta)}{\cos \theta}+\frac{m g z_{G}}{J_{x}} \frac{\operatorname{sen} \phi \operatorname{sen} \theta}{\cos \theta}-\frac{1}{J_{x}} \frac{\operatorname{sen} \theta\left(\tau_{x} \cos \psi+\tau_{y} \operatorname{sen} \psi\right)}{\cos \theta}-\frac{1}{J_{z}} \tau_{z}
\end{aligned}\right.
$$

\section{Sub-sistema não-linear}

O sistema não-linear de três equações diferenciais ordinárias de $2^{\mathrm{a}}$ ordem obtido também pode ser expresso como um sistema não-linear de seis equações diferenciais ordinárias de $1^{\mathrm{a}}$ ordem da seguinte forma:

$$
\left\{\begin{array}{l}
\dot{x}=f_{h}\left(x_{h}\right)+g_{h}\left(x_{h}\right) u_{h} \\
y_{h}=h\left(x_{h}\right)
\end{array}\right.
$$

Onde:

- $x_{h}$ é o vetor de estados do sub-sistema da haste

- $u_{h}$ é o vetor de entradas do sub-sistema da haste

- $y_{h}$ é o vetor de saídas do sub-sistema da haste

- $f_{h}$ é o vetor de funções não-lineares que descreve a dinâmica do sub-sistema da haste

- $g_{h}$ é a matriz de funções não-lineares que descreve o acoplamento das entradas do sub-sistema da haste

- $h_{h}$ é o vetor de funções não-lineares que descreve as saídas do sub-sistema da haste

Note que $x_{h}, u_{h}$ e $y_{h}$ podem ser obtidos da seguinte forma:

$$
x_{h}=\left[\begin{array}{l}
q_{h} \\
\dot{q}_{h}
\end{array}\right], \quad u_{u}=\left[u_{h}\right], \quad y_{h}=\left[q_{h}\right]
$$

Logo: 


$$
x_{h}=\left[\begin{array}{c}
\phi \\
\theta \\
\psi \\
\dot{\phi} \\
\dot{\theta} \\
\dot{\psi}
\end{array}\right], \quad u_{u}=\left[\begin{array}{c}
\tau_{x} \\
\tau_{y} \\
\tau_{z}
\end{array}\right], \quad y_{h}=\left[\begin{array}{c}
\phi \\
\theta \\
\psi
\end{array}\right]
$$

Enquanto que $f_{h}, g_{h}$ e $h_{h}$ são obtidos a partir de:

$$
f_{h}=\left[\begin{array}{c}
\dot{q}_{h} \\
-M_{h}{ }^{-1}\left[N_{h}+O_{h}\right]
\end{array}\right], \quad g_{h}=\left[\begin{array}{c}
0 \\
M_{h}{ }^{-1} P_{h}
\end{array}\right], \quad h_{h}=\left[q_{h}\right]
$$

Ou seja:

$$
f_{h}=\left[\begin{array}{c}
\dot{\phi} \\
\dot{\theta} \\
\dot{\psi} \\
\frac{J_{z} \dot{\psi} \dot{\theta}+2 J_{x} \dot{\phi} \dot{\theta} \operatorname{sen} \theta-J_{z} \dot{\phi} \dot{\theta} \operatorname{sen} \theta+m g z_{G} \operatorname{sen}}{J_{x} \cos \theta} \\
-\frac{J_{x} \dot{\phi}^{2} \cos \theta \operatorname{sen} \theta+J_{z} \dot{\phi} \cos \theta(\dot{\psi} \dot{\phi} \operatorname{sen} \theta)-m g z_{G} \cos }{J_{x} \dot{\phi} \dot{\theta}+J_{z} \dot{\psi} \dot{\theta} \operatorname{sen} \theta+J_{x} \dot{\phi} \dot{\theta} \operatorname{sen}^{2} \theta-J_{z} \dot{\phi} \dot{\theta} \operatorname{sen}^{2} \theta+m g z_{G}} \\
\frac{J_{x} \cos \theta}{0} \\
g_{h}=\left[\begin{array}{ccc}
0 & 0 \\
0 & 0 & 0 \\
0 & 0 & 0 \\
-\frac{\cos \psi}{J_{x} \cos \theta} & -\frac{\operatorname{sen} \psi}{J_{x} \cos \theta} & 0 \\
\frac{\operatorname{sen} \psi}{J_{x}} & -\frac{\cos \psi}{J_{x}} & 0 \\
-\frac{\cos \psi \operatorname{tg} \theta}{J_{x}} & -\frac{\operatorname{sen} \psi \operatorname{tg} \theta}{J_{x}} & -\frac{1}{J_{z}}
\end{array}\right] \\
h_{h}=\left[\begin{array}{c}
\phi \\
\theta \\
\psi
\end{array}\right]
\end{array}\right.
$$

O sub-sistema da haste não-linear pode ser representado conforme o diagrama de blocos da Figura 3.3.

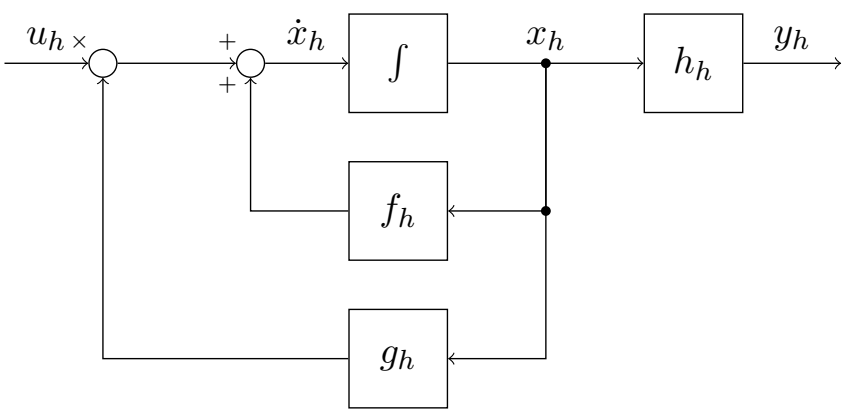

Figura 3.3: Diagrama de blocos do sub-sistema da haste 


\section{Sub-sistema linearizado}

Grande parte das teorias de projeto de sistemas de controle foram desenvolvidas para sistemas lineares, porém a grande maioria dos sistemas reais são não-lineares. Técnicas de projeto de controladores para sistemas não-lineares são complicadas, complexas e muitas delas só garantem estabilidade.

Neste caso, existem algumas técnicas para linearizar tais sistemas. Duas bastante comuns e que serão abordadas nesse trabalho são a linearização padrão e a linearização exata. A linearização exata envolve uma realimentação não-linear e, portanto, será abordada na próxima seção. Nesta seção será analisada apenas a linearização padrão.

A técnica de linearização padrão consiste em encontrar uma versão aproximada e simplificada que represente bem a dinâmica do sistema em torno de uma determinada condição nominal de operação e que seja, necessariamente, uma condição de equilíbrio.

Dessa forma, a primeira etapa para aplicar a linearização padrão consiste em determinar a condição nominal de operação do sistema. No caso do pêndulo invertido, essa tarefa é bem intuitiva. Como o objetivo é estabilizar o pêndulo na vertical, a posição e orientação nominal de operação do sistema é dada por:

$$
\left\{\begin{array}{l}
\phi_{0}=\dot{\phi}_{0}=\ddot{\phi}_{0}=0 \\
\theta_{0}=\dot{\theta}_{0}=\ddot{\theta}_{0}=0 \\
\psi_{0}=\dot{\psi}_{0}=\ddot{\psi}_{0}=0
\end{array} \rightarrow x_{0}=\left[\begin{array}{l}
0 \\
0 \\
0 \\
0 \\
0 \\
0
\end{array}\right], \quad\left\{\begin{array}{l}
\tau_{x 0}=0 \\
\tau_{y_{0}}=0 \\
\tau_{z 0}=0
\end{array} \rightarrow u_{0}=\left[\begin{array}{l}
0 \\
0 \\
0
\end{array}\right]\right.\right.
$$

Nota-se que esse sub-sistema linearizado é um sistema linear de seis equações diferenciais ordinárias de $1^{\mathrm{a}}$ ordem, podendo ser expresso da seguinte forma:

$$
\left\{\begin{array}{l}
\dot{x}_{h}=A_{h} x_{h}+B_{h} u_{h} \\
y_{h}=C_{h} x_{h}
\end{array}\right.
$$

Onde:

- $x_{h}$ é o vetor de estados do sub-sistema da haste

- $u_{h}$ é o vetor de entradas do sub-sistema da haste

- $y_{h}$ é o vetor de saídas do sistema do sub-sistema da haste

- $A_{h}$ é a matriz de estados do sub-sistema da haste

- $B_{h}$ é a matriz de entradas do sub-sistema da haste

- $C_{h}$ é o matriz de saídas do sub-sistema da haste

Os estados desse sub-sistema são os ângulos de Euler $\phi, \theta$ e $\psi$ e suas derivadas temporais $\dot{\phi}, \dot{\theta}$ e $\dot{\psi}$, as entradas são os torques úteis $\tau_{x}, \tau_{y}$ e $\tau_{z}$ e as saídas são os ângulos de Euler $\phi, \theta$ e $\psi$ : 


$$
x_{h}=\left[\begin{array}{c}
\phi \\
\theta \\
\psi \\
\dot{\phi} \\
\dot{\theta} \\
\dot{\psi}
\end{array}\right], \quad u_{h}=\left[\begin{array}{c}
\tau_{x} \\
\tau_{y} \\
\tau_{z}
\end{array}\right], \quad y_{h}=\left[\begin{array}{c}
\phi \\
\theta \\
\psi
\end{array}\right]
$$

Na linearização padrão $A_{h}, B_{h}$ e $C_{h}$ são obtidos respectivamente através dos Jacobianos de $f_{h}$ com relação a $x_{h}, g_{h} u_{h}$ com relação a $u_{h}$ e $h_{h}$ com relação a $y_{h}$, todos na condição nominal de operação:

$A_{h}=\left[\begin{array}{ccc}\left.\frac{\partial f_{h_{1}}}{\partial x_{h_{1}}}\right|_{0} & \cdots & \left.\frac{\partial f_{h_{1}}}{\partial x_{h_{n}}}\right|_{0} \\ \vdots & \ddots & \vdots \\ \left.\frac{\partial f_{h_{n}}}{\partial x_{h_{1}}}\right|_{0} & \cdots & \left.\frac{\partial f_{h_{n}}}{\partial x_{h_{n}}}\right|_{0}\end{array}\right], \quad B_{h}=\left[\begin{array}{ccc}\left.\frac{\partial g_{h} u_{h_{1}}}{\partial u_{h_{1}}}\right|_{0} & \ldots & \left.\frac{\partial g_{h} u_{h_{1}}}{\partial u_{h_{n}}}\right|_{0} \\ \vdots & \ddots & \vdots \\ \left.\frac{\partial g_{h} u_{h_{n}}}{\partial u_{h_{1}}}\right|_{0} & \cdots & \left.\frac{\partial g_{h} u_{h_{n}}}{\partial u_{h_{n}}}\right|_{0}\end{array}\right], \quad C_{h}=\left[\begin{array}{ccc}\left.\frac{\partial h_{h_{1}}}{\partial y_{h_{1}}}\right|_{0} & \ldots & \left.\frac{\partial h_{h_{1}}}{\partial y_{h_{n}}}\right|_{0} \\ \vdots & \ddots & \vdots \\ \left.\frac{\partial h_{h_{n}}}{\partial y_{h_{1}}}\right|_{0} & \cdots & \left.\frac{\partial h_{h_{n}}}{\partial y_{h_{n}}}\right|_{0}\end{array}\right]$

Logo:

$A_{h}=\left[\begin{array}{cccccc}0 & 0 & 0 & 1 & 0 & 0 \\ 0 & 0 & 0 & 0 & 1 & 0 \\ 0 & 0 & 0 & 0 & 0 & 1 \\ \frac{m g z_{G}}{J_{x}} & 0 & 0 & 0 & 0 & 0 \\ 0 & \frac{m g z_{G}}{J_{x}} & 0 & 0 & 0 & 0 \\ 0 & 0 & 0 & 0 & 0 & 0\end{array}\right], \quad B_{h}=\left[\begin{array}{ccc}0 & 0 & 0 \\ 0 & 0 & 0 \\ 0 & 0 & 0 \\ -\frac{1}{J_{x}} & 0 & 0 \\ 0 & -\frac{1}{J_{x}} & 0 \\ 0 & 0 & -\frac{1}{J_{z}}\end{array}\right], \quad C_{h}=\left[\begin{array}{cccccc}1 & 0 & 0 & 0 & 0 & 0 \\ 0 & 1 & 0 & 0 & 0 & 0 \\ 0 & 0 & 1 & 0 & 0 & 0\end{array}\right]$

O sub-sistema da haste linearizado pode ser representado conforme o diagrama de blocos da Figura 3.4 .

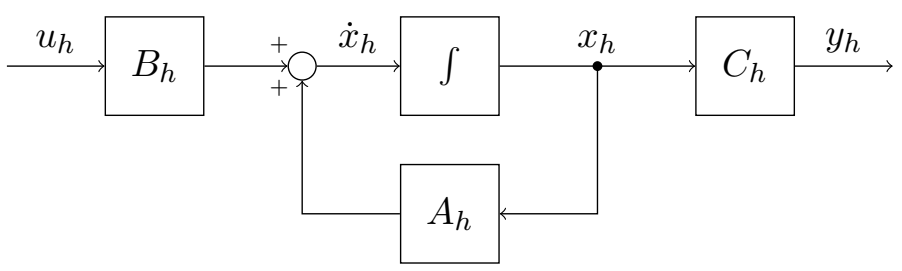

Figura 3.4: Diagrama de blocos do sub-sistema da haste linearizado (linearização padrão)

\section{Sub-sistema linearizado e simplificado}

Analizando as matrizes $A_{h}, B_{h}$ e $C_{h}$ obtidas e dado que $x_{h}=\left[\begin{array}{ll}q_{h} & \dot{q}_{h}\end{array}\right]^{T}$, percebe-se que $\dot{q}_{h}$ independe de outros estados, enquanto que $\ddot{q}_{h}$ depende apenas de $q_{h}$ e $u_{h}$. Logo, 
outra forma de representar esse mesmo sub-sistema linearizado é conforme o diagrama de blocos da Figura 3.5.

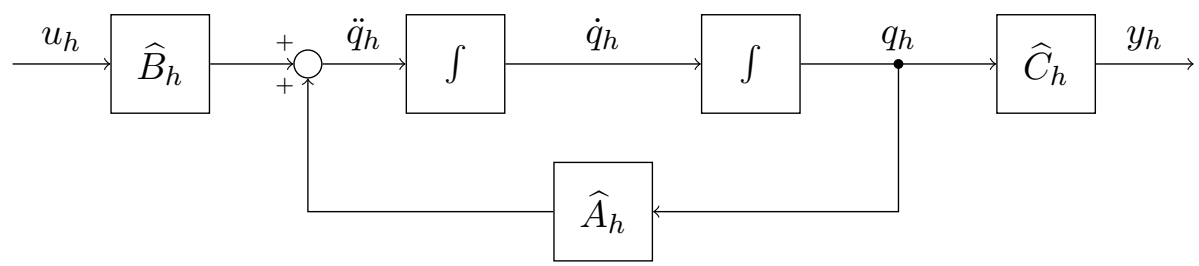

Figura 3.5: Diagrama de blocos do sub-sistema da haste linearizado (linearização padrão) e simplificado

Onde:

$$
\widehat{A}_{h}=\left[\begin{array}{cccccc}
\frac{m g z_{G}}{J_{x}} & 0 & 0 & 0 & 0 & 0 \\
0 & \frac{m g z_{G}}{J_{x}} & 0 & 0 & 0 & 0 \\
0 & 0 & 0 & 0 & 0 & 0
\end{array}\right], \quad \widehat{B}_{h}=\left[\begin{array}{ccc}
-\frac{1}{J_{x}} & 0 & 0 \\
0 & -\frac{1}{J_{x}} & 0 \\
0 & 0 & -\frac{1}{J_{z}}
\end{array}\right], \quad \widehat{C}_{h}=\left[\begin{array}{ccc}
1 & 0 & 0 \\
0 & 1 & 0 \\
0 & 0 & 1
\end{array}\right]
$$

Comparando diagramas de blocos das Figuras 3.4 e 3.5, pode-se concluir que:

$$
A_{h}=\left[\begin{array}{cc}
0 & I \\
\widehat{A}_{h}
\end{array}\right], \quad B_{h}=\left[\begin{array}{c}
0 \\
\widehat{B}_{h}
\end{array}\right], \quad C_{h}=\left[\begin{array}{ll}
\widehat{C}_{h} & 0
\end{array}\right]
$$

\subsubsection{Estabilidade}

Os pólos de um sistema fornecem o seu comportamento dinâmico (estabilidade, tempo de resposta, frequência natural, coeficiente de amortecimento, etc.). Eles podem ser obtidos a partir da equação característica do sistema que no caso do sistema composto pela haste depende apenas da matriz $A_{h}$ :

$$
\operatorname{det}\left[s I-A_{h}\right]=0
$$

Resolvendo a equação acima com os valores dos parâmetros calculados em na Seção 2.5 tem-se que:

$$
\left\{\begin{array}{l}
s_{1}=s_{2}=0 \\
s_{3}=s_{4}=6,61 \\
s_{5}=s_{6}=-6,61
\end{array}\right.
$$

Os pólos podem, então, ser representados no plano complexo conforme a Figura 3.6. 


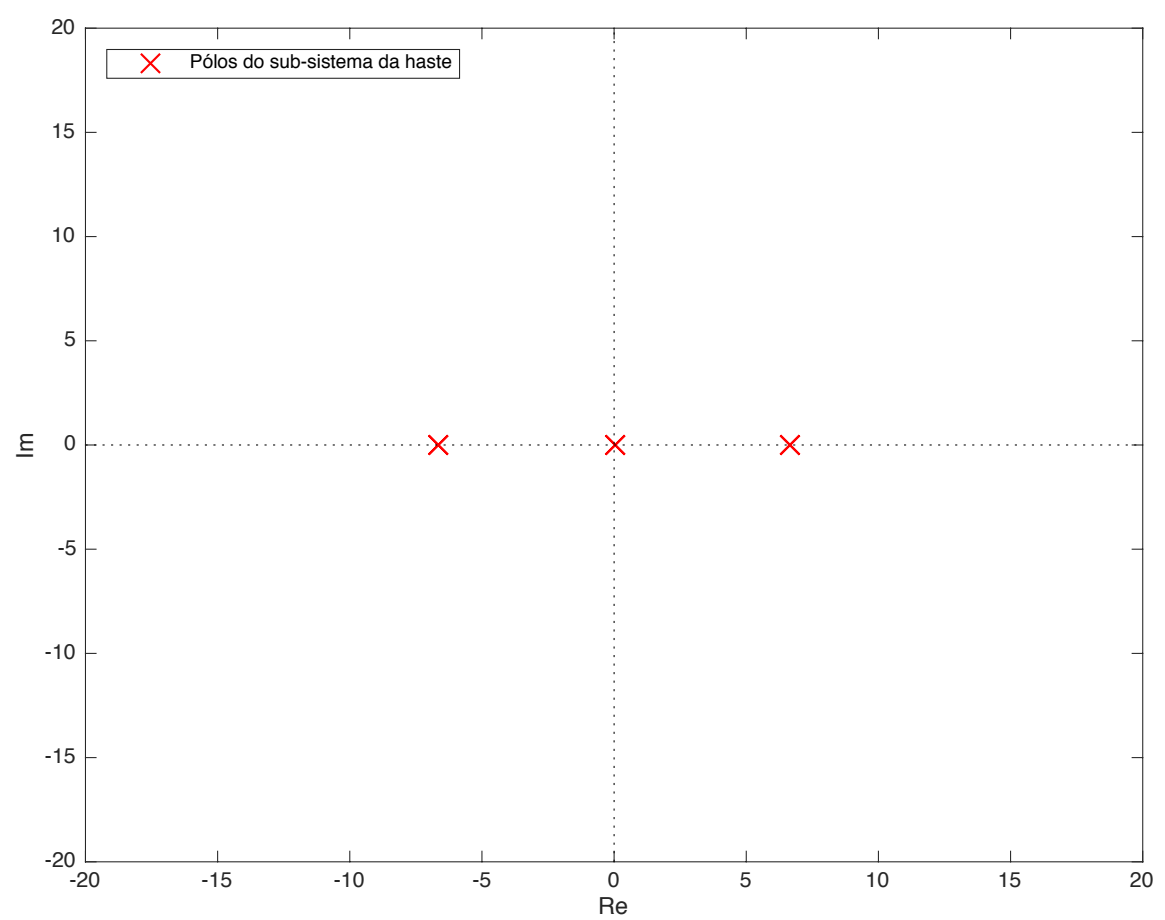

Figura 3.6: Pólos e zeros de transmissão do sistema considerado (haste)

Analisando a representação dos pólos no plano complexo, é possível concluir que o sistema em estudo é instável, pois ele possui um pólo duplo cuja parte real é positiva $\left(s_{3}\right.$ e $\left.s_{4}\right)$, além de possuir, também, um pólo duplo cuja parte real é zero $\left(s_{1}\right.$ e $\left.s_{2}\right)$.

\subsubsection{Controlabilidade e observabilidade}

Um sistema é controlável se e somente se for possível, por meio da entrada, transferir o sistema a partir de qualquer estado inicial $x\left(t_{i}\right)=x_{i}$ para qualquer outro estado final $x_{f}=x\left(t_{f}\right)$ em um tempo finito $t_{f}-t_{i} \geq 0$.

Uma maneira de testar algebricamente a controlabilidade de um sistema é por meio de sua matriz de controlabilidade. A matriz de controlabilidade de um sistema de dimensão $n$ é dada por:

$$
\mathcal{C}=\left[\begin{array}{lllll}
B & A B & A^{2} B & \ldots & A^{(n-1)} B
\end{array}\right]
$$

O sistema composto pela haste possui 6 estados $(n=6)$, portanto:

$$
\mathcal{C}_{h}=\left[\begin{array}{llllll}
B_{h} & A_{h} B_{h} & A_{h}{ }^{2} B_{h} & A_{h}{ }^{3} B_{h} & A_{h}{ }^{4} B_{h} & A_{h}{ }^{5} B_{h}
\end{array}\right]
$$


Calculando essa matriz de controlabilidade, verifica-se que a mesma possui posto ${ }^{1}$ seis, ou seja:

$$
\operatorname{rank}\left\{\mathcal{C}_{h}\right\}=6
$$

Como o posto da matriz de controlabilidade é igual à dimensão do sistema, pode-se concluir que o sistema composto pela haste é controlável.

Um sistema é observável se e somente se for possível determinar qualquer estado inicial $x\left(t_{i}\right)=x_{i}$ utilizando apenas um registro finito, $y(\tau)$ para $t_{i} \leq \tau \leq t_{f}$, de sua saída.

Uma maneira de testar algebricamente a observabilidade de um sistema é por meio de sua matriz de observabilidade. A matriz de observabilidade de um sistema de ordem $n$ é dada por:

$$
\mathcal{O}=\left[\begin{array}{c}
C \\
C A \\
C A^{2} \\
\vdots \\
C A^{(n-1)}
\end{array}\right]
$$

O sistema composto pela haste é de ordem seis $(n=6)$, logo:

$$
\mathcal{O}_{h}=\left[\begin{array}{c}
C_{h} \\
C_{h} A_{h} \\
C_{h} A_{h}{ }^{2} \\
C_{h} A_{h}{ }^{3} \\
C_{h} A_{h}{ }^{4} \\
C_{h} A_{h}{ }^{5}
\end{array}\right]
$$

Do mesmo mode que a matriz de controlabilidade, a matriz de observabilidade possui posto seis:

$$
\operatorname{rank}\left\{\mathcal{O}_{h}\right\}=6
$$

Assim, pode-se concluir que o sistema composto pela haste é observável.

\subsection{Análise da haste com as rodas de reação}

Agora, o sistema considerado será composto pela haste e rodas de reação, conforme o diagrama de blocos da Figura 3.7:

\footnotetext{
${ }^{1}$ Menor número de linhas e/ou colunas linearmente independentes
} 


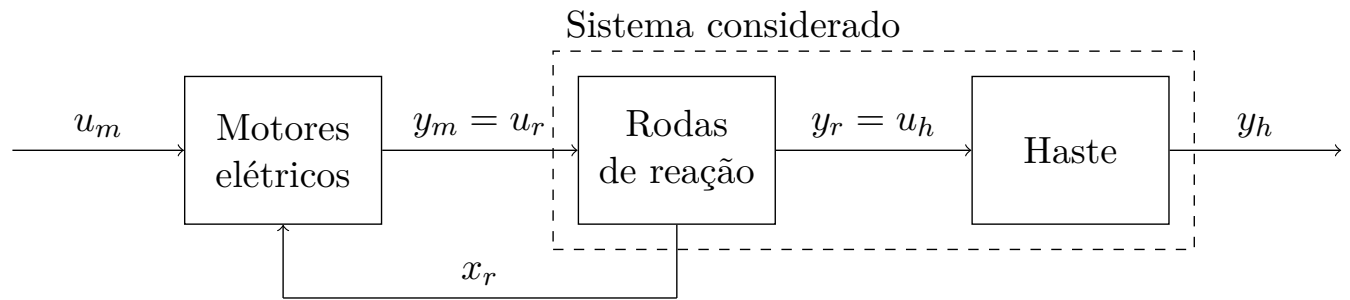

Figura 3.7: Diagrama de blocos do sistema completo e considerado (haste e rodas de reação)

Dado que o sub-sistema da haste já foi definido, torna-se necessário apenas definir o sub-sistema das rodas de reação e inclú́-lo no sistema definido na Seção 3.2.

\subsubsection{Sub-sistema das rodas de reação}

Conforme deduzido na Seção 2.3, as equações diferenciais do sub-sistema das rodas de reação são dadas por:

$$
\left\{\begin{array}{l}
\ddot{\alpha}=-\frac{b}{J_{r}} \dot{\alpha}+\frac{1}{J_{r}} \bar{\tau}_{x} \\
\ddot{\beta}=-\frac{b}{J_{r}} \dot{\beta}+\frac{1}{J_{r}} \bar{\tau}_{y} \\
\ddot{\gamma}=-\frac{b}{J_{r}} \dot{\gamma}+\frac{1}{J_{r}} \bar{\tau}_{z}
\end{array}\right.
$$

Nota-se que esse sub-sistema é um sistema linear de três equações diferenciais ordinárias de $1^{\mathrm{a}}$ ordem, podendo ser expresso da seguinte forma:

$$
\left\{\begin{array}{l}
\dot{x}_{r}=A_{r} x_{r}+B_{r} u_{r} \\
y_{r}=C_{r} x_{r}+D_{r} u_{r}
\end{array}\right.
$$

Onde:

- $x_{r}$ é o vetor de estados do sub-sistema das rodas de reação

- $u_{r}$ é o vetor de entradas do sub-sistema das rodas de reação

- $y_{r}$ é o vetor de saídas do sistema do sub-sistema das rodas de reação

- $A_{r}$ é a matriz de estados do sub-sistema das rodas de reação

- $B_{r}$ é a matriz de entradas do sub-sistema das rodas de reação

- $C_{r}$ é o matriz de saídas do sub-sistema das rodas de reação

- $D_{r}$ é a matriz de alimentação direta do sub-sistema das rodas de reação

Os estados desse sub-sistema são as velocidades angulares das rodas de reação $\dot{\alpha}, \dot{\beta}$ e $\dot{\gamma}$, as entradas são os torques $\bar{\tau}_{x}, \bar{\tau}_{y}$ e $\bar{\tau}_{z}$ e as saídas são os toques úteis $\tau_{x}$, $\tau_{y}$ e $\tau_{z}$ :

$$
x_{r}=\left[\begin{array}{c}
\dot{\alpha} \\
\dot{\beta} \\
\dot{\gamma}
\end{array}\right], \quad u_{r}=\left[\begin{array}{c}
\bar{\tau}_{x} \\
\bar{\tau}_{y} \\
\bar{\tau}_{z}
\end{array}\right], \quad y_{r}=\left[\begin{array}{c}
\tau_{x} \\
\tau_{y} \\
\tau_{z}
\end{array}\right]
$$

Os torques úteis são dados por: 


$$
\left\{\begin{array}{l}
\tau_{x}=J_{r} \ddot{\alpha} \\
\tau_{y}=J_{r} \ddot{\beta} \\
\tau_{z}=J_{r} \ddot{\gamma}
\end{array}\right.
$$

Isolando os termos $J_{r} \ddot{\alpha}, J_{r} \ddot{\beta}$ e $J_{r} \ddot{\gamma}$ na Equação (3.36), tem-se que:

$$
\left\{\begin{array}{l}
\tau_{x}=\bar{\tau}_{x}-b \dot{\alpha} \\
\tau_{y}=\bar{\tau}_{y}-b \dot{\beta} \\
\tau_{z}=\bar{\tau}_{z}-b \dot{\gamma}
\end{array}\right.
$$

Dessa forma, tem-se que:

$$
A_{r}=\left[\begin{array}{ccc}
-\frac{b}{J_{r}} & 0 & 0 \\
0 & -\frac{b}{J_{r}} & 0 \\
0 & 0 & -\frac{b}{J_{r}}
\end{array}\right], B_{r}=\left[\begin{array}{ccc}
\frac{1}{J_{r}} & 0 & 0 \\
0 & \frac{1}{J_{r}} & 0 \\
0 & 0 & \frac{1}{J_{r}}
\end{array}\right], C_{r}=\left[\begin{array}{ccc}
-b & 0 & 0 \\
0 & -b & 0 \\
0 & 0 & -b
\end{array}\right], D_{r}=\left[\begin{array}{ccc}
1 & 0 & 0 \\
0 & 1 & 0 \\
0 & 0 & 1
\end{array}\right]
$$

O diagrama de blocos que representa o sub-sistema das rodas de reação pode ser observado na Figura 3.8:

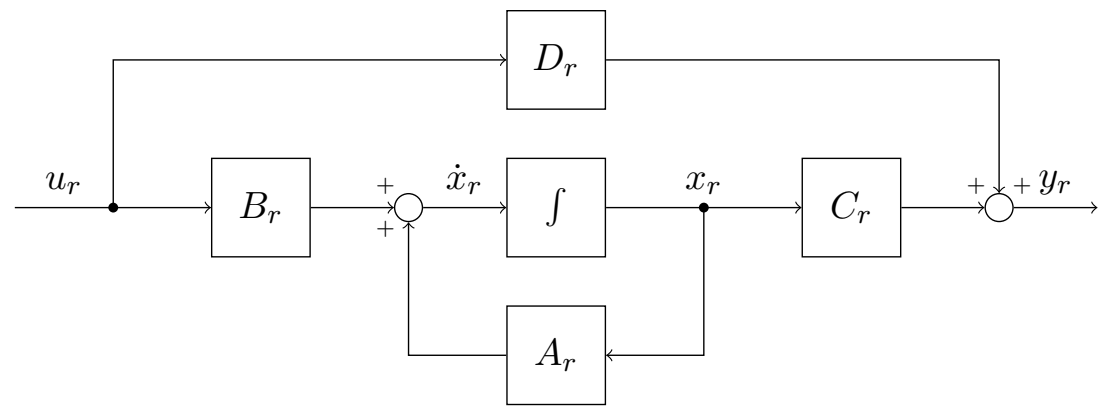

Figura 3.8: Diagrama de blocos do sub-sistema das rodas de reação

\subsubsection{Inclusão das rodas de reação no sistema}

Agora que sub-sistema das rodas de reação já foi definido, o sistema considerado passa a ser o sub-sistema das rodas de reação em série com o sub-sistema da haste, conforme a Figura 3.9. 


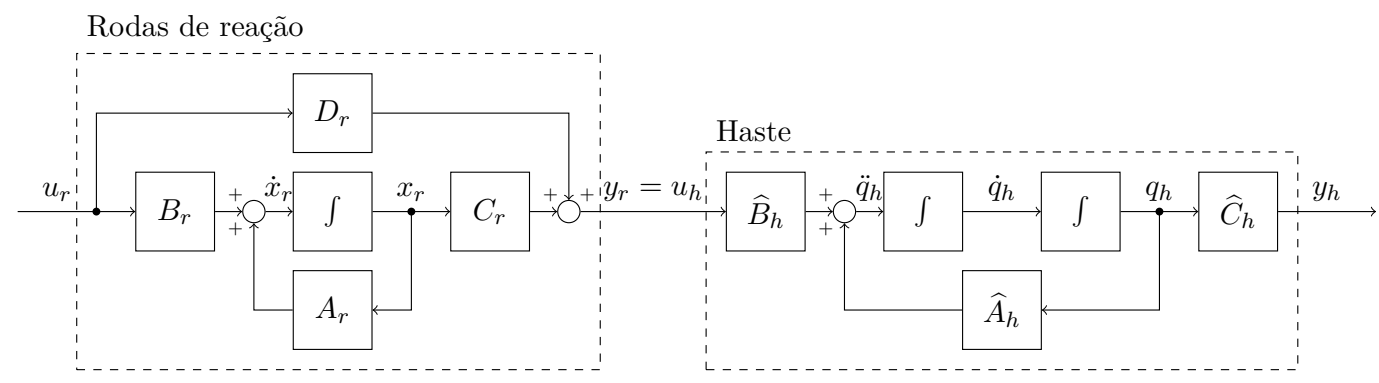

Figura 3.9: Diagrama de blocos do sistema considerado (haste e rodas de reação)

Os estados desse sistema são os estados dos dois sub-sistemas, enquanto que as entradas são as mesmas que as do sub-sistema das rodas de reação e as saídas são as mesmas que as do sub-sistema da haste:

$$
x_{r h}=\left[\begin{array}{c}
x_{h} \\
x_{r}
\end{array}\right]=\left[\begin{array}{c}
\phi \\
\theta \\
\psi \\
\dot{\phi} \\
\dot{\theta} \\
\dot{\psi} \\
\dot{\alpha} \\
\dot{\beta} \\
\dot{\gamma}
\end{array}\right], \quad u_{r h}=u_{r}=\left[\begin{array}{c}
\bar{\tau}_{x} \\
\bar{\tau}_{y} \\
\bar{\tau}_{z}
\end{array}\right], \quad y_{r h}=y_{h}=\left[\begin{array}{c}
\phi \\
\theta \\
\psi
\end{array}\right] \text {, }
$$

Dessa maneira, esse sistema pode ser representado de modo mais simplificado conforme a Figura 3.10.

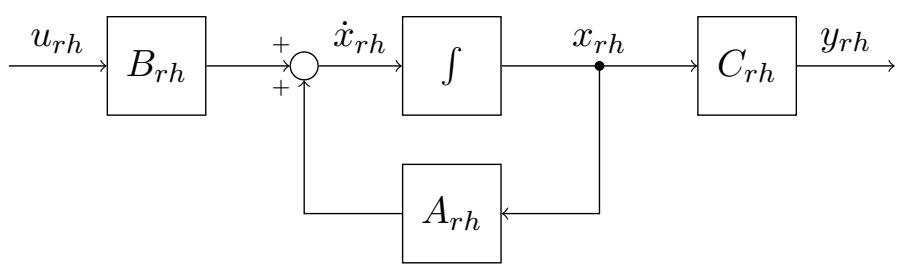

Figura 3.10: Diagrama de blocos simplificado do sistema considerado (haste e rodas de reação)

Onde:

$$
A_{r h}=\left[\begin{array}{cc}
A_{h} & B_{h} C_{r} \\
0 & A_{r}
\end{array}\right], \quad B_{r h}=\left[\begin{array}{c}
B_{h} D_{r} \\
B_{r}
\end{array}\right], \quad C_{r h}=\left[\begin{array}{ll}
C_{h} & D_{h} C_{r}
\end{array}\right]
$$

Logo: 


$$
\begin{aligned}
& A_{r h}=\left[\begin{array}{ccccccccc}
0 & 0 & 0 & 1 & 0 & 0 & 0 & 0 & 0 \\
0 & 0 & 0 & 0 & 1 & 0 & 0 & 0 & 0 \\
0 & 0 & 0 & 0 & 0 & 1 & 0 & 0 & 0 \\
\frac{m g z_{G}}{J_{x}} & 0 & 0 & 0 & 0 & 0 & \frac{b}{J_{x}} & 0 & 0 \\
0 & \frac{m g z_{G}}{J_{x}} & 0 & 0 & 0 & 0 & 0 & \frac{b}{J_{x}} & 0 \\
0 & 0 & 0 & 0 & 0 & 0 & 0 & 0 & \frac{b}{J_{z}} \\
0 & 0 & 0 & 0 & 0 & 0 & -\frac{b}{J_{r}} & 0 & 0 \\
0 & 0 & 0 & 0 & 0 & 0 & 0 & -\frac{b}{J_{r}} & 0 \\
0 & 0 & 0 & 0 & 0 & 0 & 0 & 0 & -\frac{b}{J_{r}}
\end{array}\right] \\
& B_{r h}=\left[\begin{array}{ccc}
0 & 0 & 0 \\
0 & 0 & 0 \\
0 & 0 & 0 \\
-\frac{1}{J_{x}} & 0 & 0 \\
0 & -\frac{1}{J_{x}} & 0 \\
0 & 0 & -\frac{1}{J_{z}} \\
\frac{1}{J_{r}} & 0 & 0 \\
0 & \frac{1}{J_{r}} & 0 \\
0 & 0 & \frac{1}{J_{r}}
\end{array}\right] \\
& C_{r h}=\left[\begin{array}{lllllllll}
1 & 0 & 0 & 0 & 0 & 0 & 0 & 0 & 0 \\
0 & 1 & 0 & 0 & 0 & 0 & 0 & 0 & 0 \\
0 & 0 & 1 & 0 & 0 & 0 & 0 & 0 & 0
\end{array}\right]
\end{aligned}
$$

\subsubsection{Estabilidade}

A nova equação característica do sistema passa a ser dada por:

$$
\operatorname{det}\left[s I-A_{r h}\right]=0
$$

Portanto, os novos pólos do sistema são:

$$
\left\{\begin{array}{l}
s_{1}=s_{2}=0 \\
s_{3}=s_{4}=6,61 \\
s_{5}=s_{6}=-6,61 \\
s_{7}=s_{8}=s_{9}=-0,18
\end{array}\right.
$$

Percebe-se que são os mesmos 6 pólos já existentes $\left(s_{1}, s_{2}, s_{3}, s_{4}, s_{5}\right.$ e $\left.s_{6}\right)$, porém com a adição de mais 3 pólos devidos ao sub-sistema das rodas de reação $\left(s_{7}, s_{8}\right.$ e $\left.s_{9}\right)$.

Esses pólos podem ser representados no plano complexo conforme a Figura 3.11. 


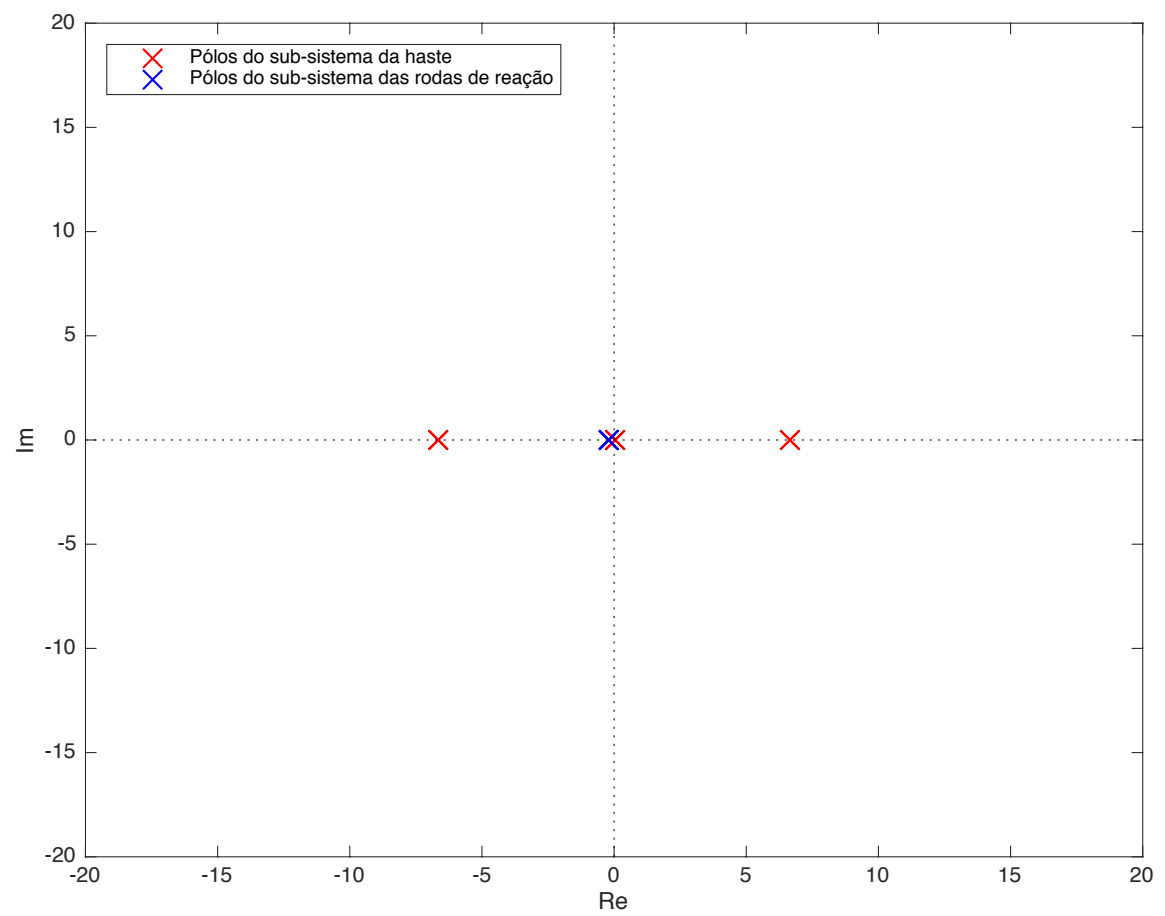

Figura 3.11: Pólos e zeros de transmissão do sistema considerado (haste e rodas de reação)

Analisando a representação dos pólos no plano complexo, é possível concluir que o sistema em estudo continua sendo instável pois ele ainda possui os mesmos pólos do sub-sistema da haste (o qual é instável). Os novos pólos do sub-sistema das rodas de reação $\left(s_{7}, s_{8}\right.$ e $\left.s_{9}\right)$, entretanto, não adicionam mais instabilidades ao sistema, pois suas partes reais são todas negativas.

\subsubsection{Controlabilidade e observabilidade}

O sistema com a haste e as rodas de reação possui 9 estados. Dessa forma, a nova matriz de controlabilidade passa a ser dada por:

$$
\mathcal{C}_{r h}=\left[\begin{array}{lllll}
B_{r h} & A_{r h} B_{r h} & A_{r h}{ }^{2} B_{r h} & \ldots & A_{r h}{ }^{8} B_{r h}
\end{array}\right]
$$

O posto dessas matriz é oito:

$$
\operatorname{rank}\left\{\mathcal{C}_{r h}\right\}=8
$$

O posto da matriz de controlabilidade não é igual à dimensão do sistema. Isso significa que a inclusão das rodas de reação faz com que um estado do sistema perca a 
sua controlabilidade. Entretanto, conforme será visto adiante, esse estado que perde sua controlabilidade não é de interesse para o controle e, portanto, será possível controlar o pêndulo mesmo assim.

Já a matriz de observabilidade passa a ser dada por:

$$
\mathcal{O}_{r h}=\left[\begin{array}{c}
C_{r h} \\
C_{r h} A_{r h} \\
C_{r h} A_{r h}{ }^{2} \\
\vdots \\
C_{r h} A_{r h}{ }^{8}
\end{array}\right]
$$

Essa matriz possui posto nove:

$$
\operatorname{rank}\left\{\mathcal{O}_{r h}\right\}=9
$$

Como o posto da matriz de observabilidade é igual à dimensão do sistema, a adição das rodas de reação faz com que o sistema continue sendo completamente observável.

\subsection{Análise da haste com as rodas de reação e motores elétricos}

Por fim, o sistema considerado será o sistema completo, composto pelos sub-sistemas da haste, das rodas de reação e dos motores elétricos, conforme o diagrama de blocos da Figura 3.12:

Sistema considerado

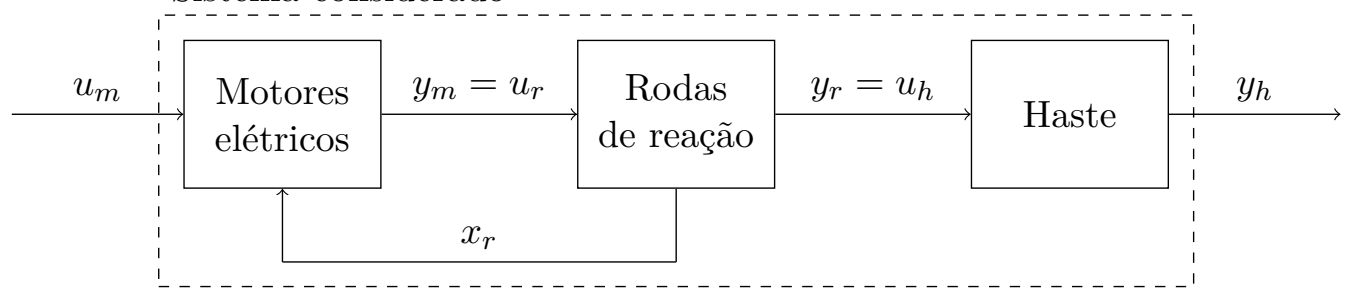

Figura 3.12: Diagrama de blocos do sistema completo e considerado (haste, rodas de reação e motores elétricos)

Dado que tanto o sub-sistema da haste como o das rodas de reação já foram definidos, torna-se necessário apenas definir o sub-sistema dos motores elétricos.

\subsubsection{Sub-sistema dos motores elétricos}

Conforme deduzido na Seção 2.4, as equações diferenciais do sub-sistema dos motores elétricos são dadas por: 


$$
\left\{\begin{array}{l}
\dot{\bar{\tau}_{x}}=-\frac{R}{L} \bar{\tau}_{x}-\frac{k_{m} k_{\tau}}{L} \dot{\alpha}+\frac{k_{\tau}}{L} e_{x} \\
\dot{\dot{\tau}_{y}}=-\frac{R}{L} \bar{\tau}_{y}-\frac{k_{m} k_{\tau}}{L} \dot{\beta}+\frac{k_{\tau}}{L} e_{y} \\
\dot{\bar{\tau}_{z}}=-\frac{R}{L} \bar{\tau}_{z}-\frac{k_{m} k_{\tau}}{L} \dot{\gamma}+\frac{k_{\tau}}{L} e_{z}
\end{array}\right.
$$

Nota-se que esse sub-sistema é um sistema linear de três equações diferenciais ordinárias de primeira ordem, podendo ser expresso da seguinte forma:

$$
\left\{\begin{array}{l}
\dot{x}_{m}=A_{m} x_{m}+B_{m} u_{m}+E_{m} x_{r} \\
y_{m}=C_{m} x_{m}
\end{array}\right.
$$

Onde:

- $x_{m}$ é o vetor de estados do sub-sistema dos motores elétricos

- $u_{m}$ é o vetor de entradas do sub-sistema dos motores elétricos

- $x_{r}$ é o vetor de estados do sub-sistema das rodas de reação

- $y_{m}$ é o vetor de saídas do sistema do sub-sistema dos motores elétricos

- $A_{m}$ é a matriz de estados do sub-sistema dos motores elétricos

- $B_{m}$ é a matriz de entradas do sub-sistema dos motores elétricos

- $C_{m}$ é o matriz de saídas do sub-sistema dos motores elétricos

- $E_{m}$ é a matriz de entradas (realimentadas) do sub-sistema dos motores elétricos

Os estados desse sub-sistema são os torques $\bar{\tau}_{x}, \bar{\tau}_{y}$ e $\bar{\tau}_{z}$, as entradas são as tensões $e_{x}, e_{y}$ e $e_{z}$ e as velocidades angulares das rodas de reação $\dot{\alpha}, \dot{\beta}$ e $\dot{\gamma}$ (que são os estados do sub-sistema dos motores elétricos) e as saídas são os torques $\bar{\tau}_{x}, \bar{\tau}_{y}$ e $\bar{\tau}_{z}$ :

$$
x_{m}=\left[\begin{array}{c}
\bar{\tau}_{x} \\
\bar{\tau}_{y} \\
\bar{\tau}_{z}
\end{array}\right], \quad u_{m}=\left[\begin{array}{c}
e_{x} \\
e_{y} \\
e_{z}
\end{array}\right], \quad x_{r}=\left[\begin{array}{c}
\dot{\alpha} \\
\dot{\beta} \\
\dot{\gamma}
\end{array}\right], \quad y_{m}=\left[\begin{array}{c}
\bar{\tau}_{x} \\
\bar{\tau}_{y} \\
\bar{\tau}_{z}
\end{array}\right]
$$

Dessa forma, tem-se que:

$$
\begin{gathered}
A_{m}=\left[\begin{array}{ccc}
-\frac{R}{L} & 0 & 0 \\
0 & -\frac{R}{L} & 0 \\
0 & 0 & -\frac{R}{L}
\end{array}\right], B_{m}=\left[\begin{array}{ccc}
\frac{k_{\tau}}{L} & 0 & 0 \\
0 & \frac{k_{\tau}}{L} & 0 \\
0 & 0 & \frac{k_{\tau}}{L}
\end{array}\right], C_{m}=\left[\begin{array}{ccc}
1 & 0 & 0 \\
0 & 1 & 0 \\
0 & 0 & 1
\end{array}\right] \\
E_{m}=\left[\begin{array}{ccc}
-\frac{k_{m} k_{\tau}}{L} & 0 & 0 \\
0 & -\frac{k_{m} k_{\tau}}{L} & 0 \\
0 & 0 & -\frac{k_{m} k_{\tau}}{L}
\end{array}\right]
\end{gathered}
$$

O diagrama de blocos que representa o sub-sistema dos motores elétricos pode ser observado na Figura 3.13: 


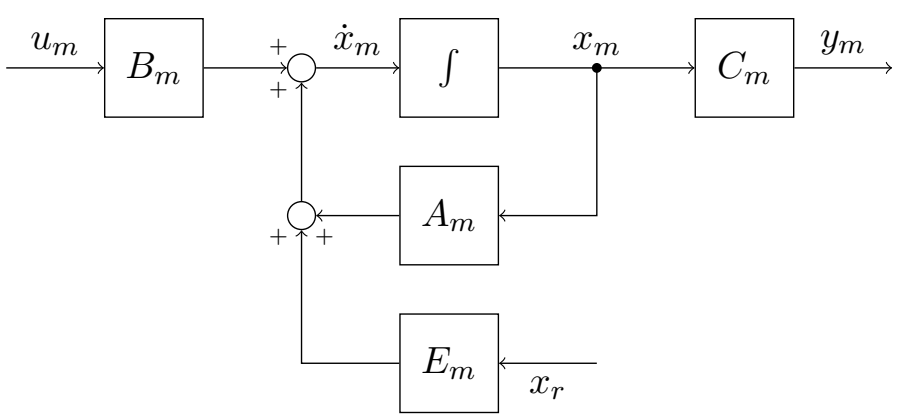

Figura 3.13: Diagrama de blocos do sub-sistema dos motores elétricos

\subsubsection{Inclusão dos motores elétricos no sistema}

Após a definição do sub-sistema dos motores elétricos, o sistema considerado passa a ser o sub-sistema dos motores elétricos em série com o sub-sistema das rodas de reação em série com o sub-sistema da haste, conforme a Figura 3.9.

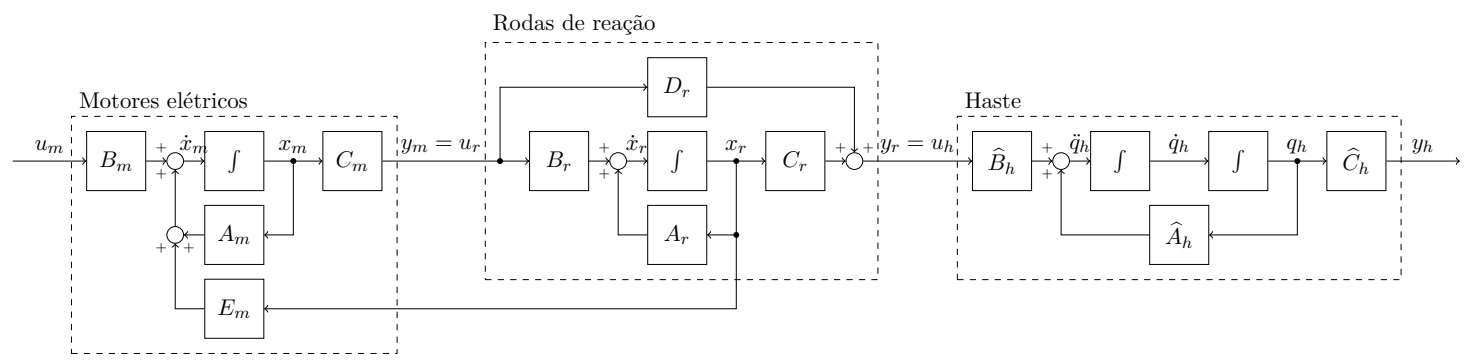

Figura 3.14: Diagrama de blocos do sistema considerado (haste, rodas de reação e motores elétricos)

Os estados desse sistema são os estados dos três sub-sistemas, enquanto que as entradas são as mesmas que as do sub-sistema dos motores elétricos e as saídas são as mesmas que as do sub-sistema da haste: 


$$
x_{m r h}=\left[\begin{array}{c}
x_{h} \\
x_{r} \\
x_{m}
\end{array}\right]=\left[\begin{array}{c}
\phi \\
\theta \\
\psi \\
\dot{\phi} \\
\dot{\theta} \\
\dot{\psi} \\
\dot{\alpha} \\
\dot{\beta} \\
\dot{\gamma} \\
\bar{\tau}_{x} \\
\bar{\tau}_{y} \\
\bar{\tau}_{z}
\end{array}\right], \quad u_{m r h}=u_{m}=\left[\begin{array}{c}
e_{x} \\
e_{y} \\
e_{z}
\end{array}\right], \quad y_{m r h}=y_{h}=\left[\begin{array}{c}
\phi \\
\theta \\
\psi
\end{array}\right],
$$

Dessa maneira, uma forma mais simplificada de representar esse sistema é conforme a Figura 3.15.

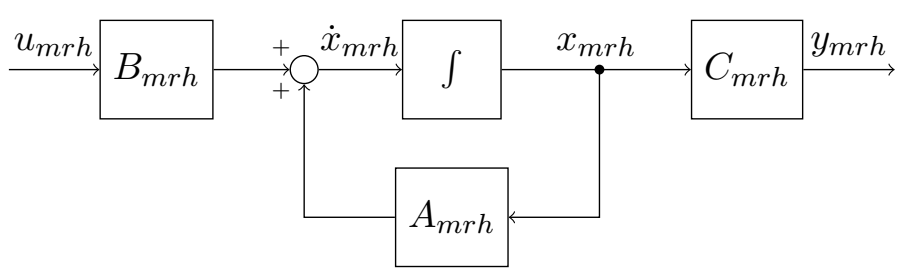

Figura 3.15: Diagrama de blocos simplificado do sistema considerado (haste, rodas de reação e motores elétricos)

Onde:

$A_{m r h}=\left[\begin{array}{ccc}A_{h} & B_{h} C_{r} & B_{h} D_{r} C_{m} \\ 0 & A_{r} & B_{r} C_{m} \\ 0 & E_{m} & A_{m}\end{array}\right], \quad B_{m r h}=\left[\begin{array}{c}B_{h} D_{r} D_{m} \\ B_{r} D_{m} \\ B_{m}\end{array}\right], \quad C_{m r h}=\left[\begin{array}{lll}C_{h} & D_{h} C_{r} & D_{h} D_{r} C_{m}\end{array}\right]$

Logo: 


$$
A_{m r h}=\left[\begin{array}{cccccccccccc}
0 & 0 & 0 & 1 & 0 & 0 & 0 & 0 & 0 & 0 & 0 & 0 \\
0 & 0 & 0 & 0 & 1 & 0 & 0 & 0 & 0 & 0 & 0 & 0 \\
0 & 0 & 0 & 0 & 0 & 1 & 0 & 0 & 0 & 0 & 0 & 0 \\
\frac{m g z_{G}}{J_{x}} & 0 & 0 & 0 & 0 & 0 & \frac{b}{J_{x}} & 0 & 0 & -\frac{1}{J_{x}} & 0 & 0 \\
0 & \frac{m g z_{G}}{J_{x}} & 0 & 0 & 0 & 0 & 0 & \frac{b}{J_{x}} & 0 & 0 & -\frac{1}{J_{x}} & 0 \\
0 & 0 & 0 & 0 & 0 & 0 & 0 & 0 & \frac{b}{J_{z}} & 0 & 0 & -\frac{1}{J_{z}} \\
0 & 0 & 0 & 0 & 0 & 0 & -\frac{b}{J_{r}} & 0 & 0 & \frac{1}{J_{r}} & 0 & 0 \\
0 & 0 & 0 & 0 & 0 & 0 & 0 & -\frac{b}{J_{r}} & 0 & 0 & \frac{1}{J_{r}} & 0 \\
0 & 0 & 0 & 0 & 0 & 0 & 0 & 0 & -\frac{b}{J_{r}} & 0 & 0 & \frac{1}{J_{r}} \\
0 & 0 & 0 & 0 & 0 & 0 & -\frac{k_{m} k_{\tau}}{L} & 0 & 0 & -\frac{R}{L} & 0 & 0 \\
0 & 0 & 0 & 0 & 0 & 0 & 0 & -\frac{k_{m} k_{\tau}}{L} & 0 & 0 & -\frac{R}{L} & 0 \\
0 & 0 & 0 & 0 & 0 & 0 & 0 & 0 & -\frac{k_{m} k_{\tau}}{L} & 0 & 0 & -\frac{R}{L}
\end{array}\right]
$$

\subsubsection{Estabilidade}

A equação característica do sistema passa a ser dada por:

$$
\operatorname{det}\left[s I-A_{m r h}\right]=0
$$

Portanto, os pólos do sistema passam a ser:

$$
\left\{\begin{array}{l}
s_{1}=s_{2}=0 \\
s_{3}=s_{4}=6,61 \\
s_{5}=s_{6}=-6,61 \\
s_{7}=s_{8}=s_{9}=-1,95 \\
s_{10}=s_{11}=s_{12}=-164,89
\end{array}\right.
$$


Percebe-se que os três pólos referentes ao sub-sistema das rodas de reação $\left(s_{7}, s_{8}\right.$ e $s_{9}$ ) foram ligeiramente modificados devido à realimentação dos estados do sub-sistema das rodas de reação no sub-sistema dos motores elétricos. Além disso, outros três pólos $\left(s_{10}, s_{11}\right.$ e $\left.s_{12}\right)$ foram adicionados ao sistema, referentes ao sub-sistema dos motores elétricos.

Esses pólos podem ser representados no plano complexo conforme a Figura 3.16.

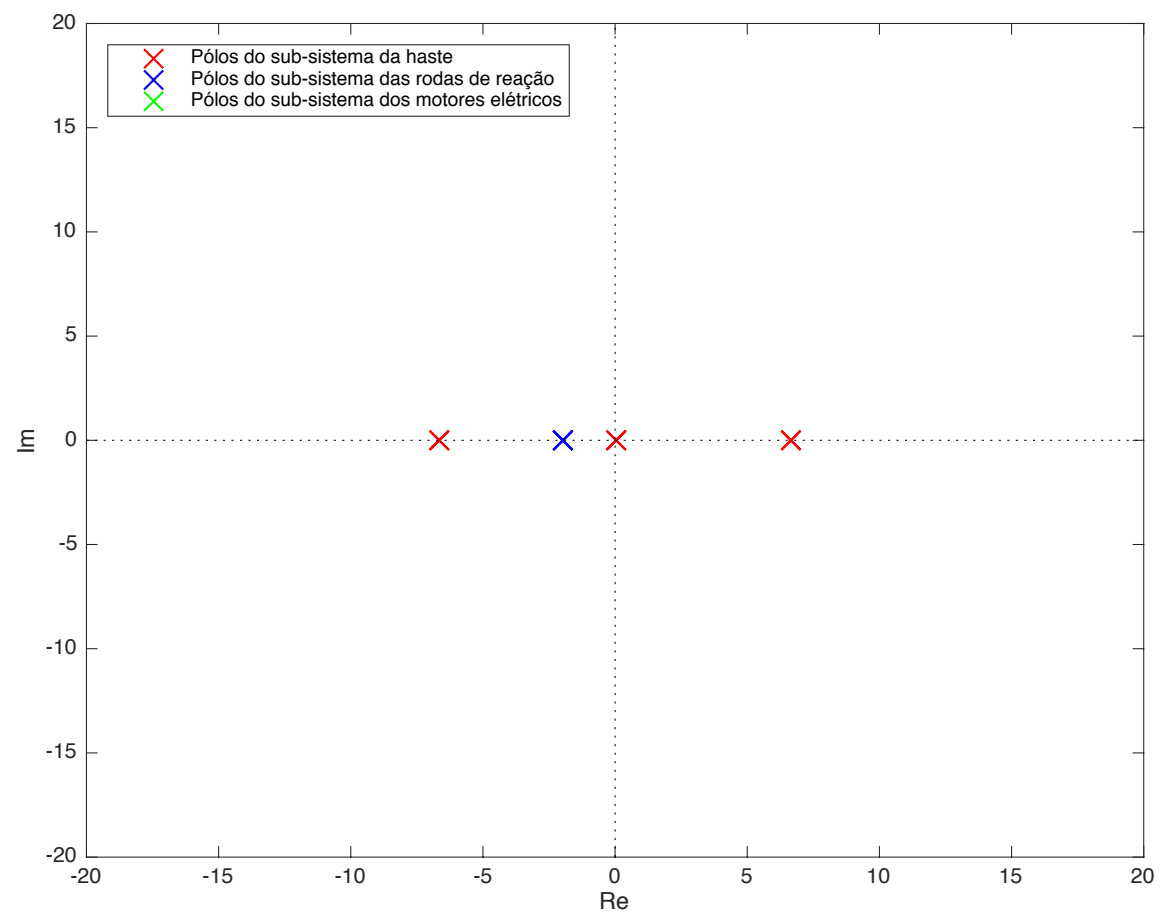

Figura 3.16: Pólos e zeros de transmissão do sistema considerado (haste, rodas de reação e motores elétricos)

É possível observar com clareza a alteração dos pólos do sub-sistema das rodas de reação $\left(s_{7}, s_{8}\right.$ e $\left.s_{9}\right)$. Entretanto, os pólos do sub-sistema dos motores elétricos não aparecem na imagem $\left(s_{10}, s_{11}\right.$ e $\left.s_{12}\right)$. Isso se deve ao fato de estarem fora da escala dessa imagem, conforme pode ser observado na Figura 3.17. 


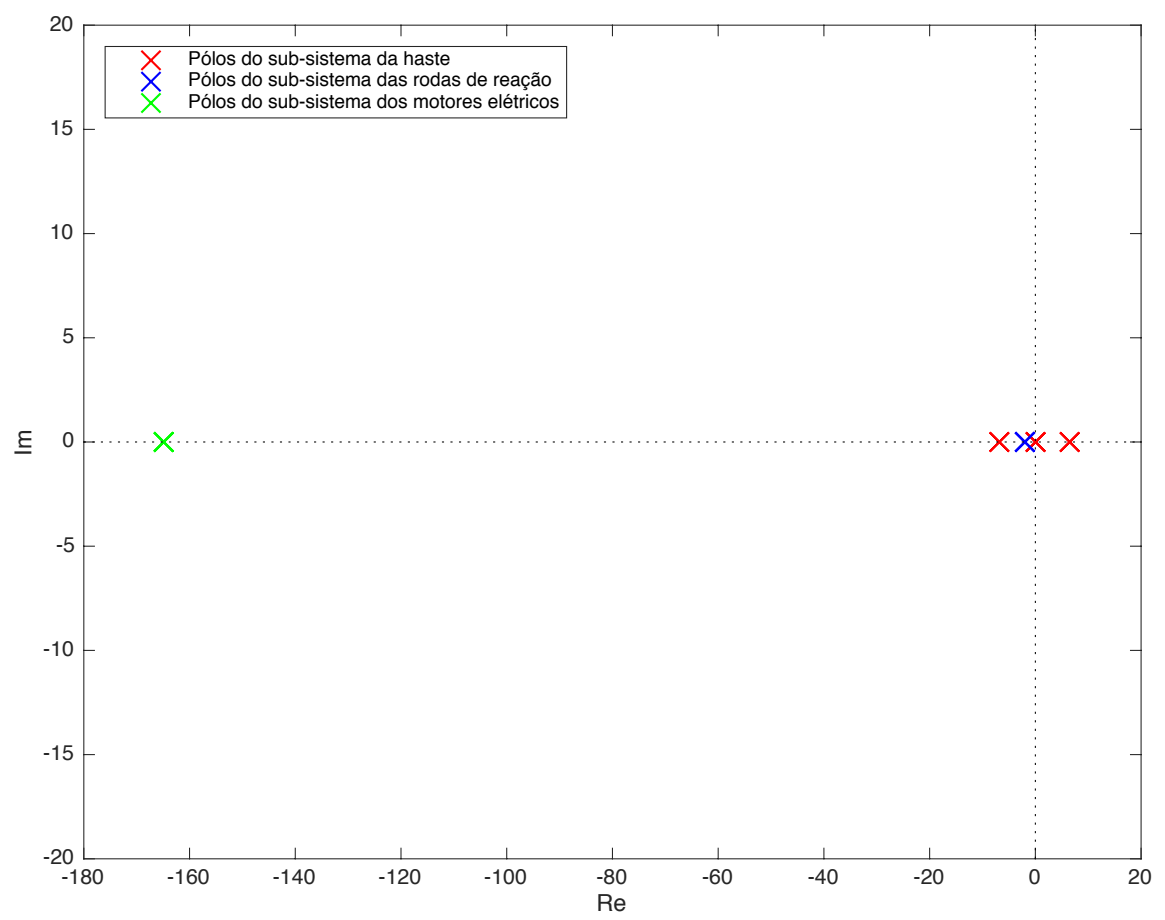

Figura 3.17: Pólos e zeros de transmissão do sistema considerado (haste, rodas de reação e motores elétricos) 2

O fato dos pólos do sub-sistema dos motores elétricos estarem tão à esquerda do eixo imaginário faz com que eles possam ser desprezados. Em outras palavras, a dinâmica dos motores elétricos é tão rápida, quando comparada à dinâmica da haste e das rodas de reação, que ela praticamente não influencia na dinâmica do sistema como um todo. Portanto, os estados dos motores elétricos não serão realimentados na lei de controle.

\subsubsection{Controlabilidade e observabilidade}

O sistema com a haste, as rodas de reação e os motores elétricos possui doze estados. Dessa forma, a matriz de controlabilidade passa a ser dada por:

$$
\mathcal{C}_{m r h}=\left[\begin{array}{lllll}
B_{m r h} & A_{m r h} B_{m r h} & A_{m r h}{ }^{2} B_{m r h} & \ldots & A_{m r h}{ }^{11} B
\end{array}\right]
$$

O posto dessas matriz é três:

$$
\operatorname{rank}\left\{\mathcal{C}_{m r h}\right\}=3
$$

Isso significa que a inclusão dos motores elétricos faz com que apenas três estados continuem a ser controláveis. Teoricamente, isto se deve a realimentação dos estados 
do sub-sistema das rodas de reação no sub-sistema dos motores elétricos. No entanto, como essa realimentação é praticamente desprezível, na prática os estados continuam a ser controláveis.

A matriz de observabilidade passa a ser dada por:

$$
\mathcal{O}_{m r h}=\left[\begin{array}{c}
C_{m r h} \\
C_{m r h} A_{m r h} \\
C_{m r h} A_{m r h}{ }^{2} \\
\vdots \\
C_{m r h} A_{m r h}{ }^{11}
\end{array}\right]
$$

Essa matriz possui posto cinco:

$$
\operatorname{rank}\left\{\mathcal{O}_{m r h}\right\}=5
$$

Isso significa que apenas cinco estados do sistema são observáveis. Isto não interfere no desenvolvimento do trabalho, pois não está previsto projetar um observador de estados.

\subsection{Desacoplamento de canais}

É interessante notar que, no sistema com linearização padrão, os canais estão totalmente desacoplados entre si, ou seja, a dinâmica do ângulo $\phi$ depende apenas da entrada $e_{x}$, enquanto que a dinâmica do ângulo $\theta$ depende apenas de $e_{y}$ e $\psi$ de $e_{z}$.

É possível, então, representar o sistema conforme o diagrama de blocos da Figura 3.18 . 


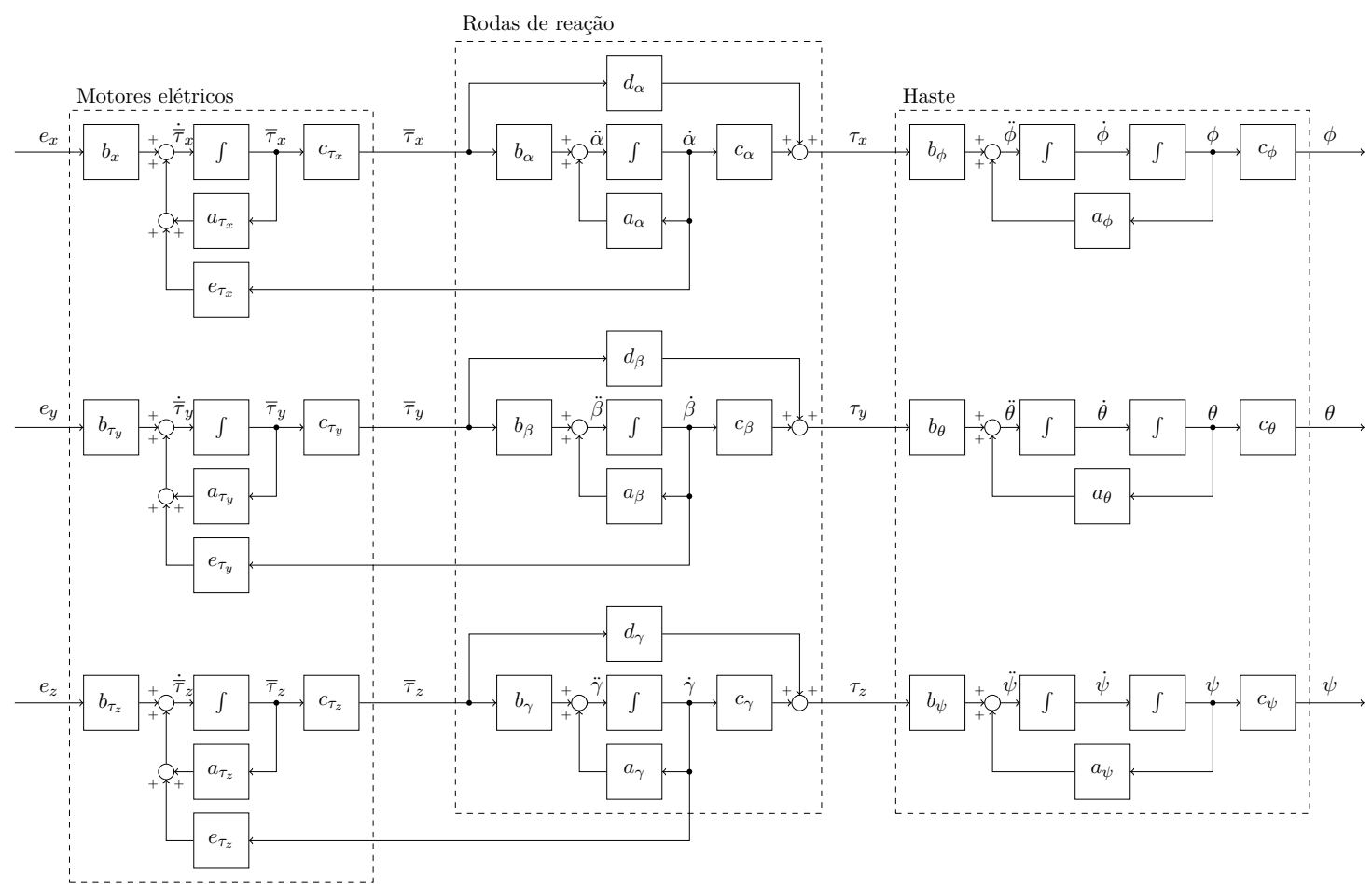

Figura 3.18: Diagrama de blocos desacoplado do sistema considerado (haste, rodas de reação e motores elétricos)

Onde:

$$
\left\{\begin{array} { l } 
{ a _ { \tau _ { x } } = - \frac { R } { L } } \\
{ b _ { \tau _ { x } } = \frac { k _ { \tau } } { L } } \\
{ c _ { \tau _ { x } } = 1 } \\
{ e _ { \tau _ { x } } = - \frac { k _ { m } k _ { \tau } } { L } } \\
{ a _ { \alpha } = - \frac { b } { J _ { r } } } \\
{ b _ { \alpha } = \frac { 1 } { J _ { r } } } \\
{ c _ { \alpha } = - b } \\
{ d _ { \alpha } = 1 } \\
{ a _ { \phi } = \frac { m g z _ { G } } { J _ { x } } } \\
{ b _ { \phi } = \frac { 1 } { J _ { x } } } \\
{ c _ { \phi } = 1 }
\end{array} \quad \left\{\begin{array}{l}
a_{\tau_{y}}=-\frac{R}{L} \\
b_{\tau_{y}}=\frac{k_{\tau}}{L} \\
c_{\tau_{y}}=1 \\
e_{\tau_{y}}=-\frac{k_{m} k_{\tau}}{L} \\
a_{\beta}=-\frac{b}{J_{r}} \\
b_{\beta}=\frac{1}{J_{r}} \\
c_{\beta}=-b \\
d_{\beta}=1 \\
a_{\theta}=\frac{m g z_{G}}{J_{x}} \\
b_{\theta}=\frac{1}{J_{x}} \\
c_{\theta}=1
\end{array} \quad, \quad\left\{\begin{array}{l}
a_{\tau_{z}}=-\frac{R}{L} \\
b_{\tau_{z}}=\frac{k_{\tau}}{L} \\
c_{\tau_{z}}=1 \\
e_{\tau_{z}}=-\frac{k_{m} k_{\tau}}{L} \\
a_{\gamma}=-\frac{b}{J_{r}} \\
b_{\gamma}=\frac{1}{J_{r}} \\
c_{\gamma}=-b \\
d_{\gamma}=1 \\
a_{\psi}=0 \\
b_{\psi}=\frac{1}{J_{r}} \\
c_{\psi}=1
\end{array}\right.\right.\right.
$$

É importante ressaltar que o sistema com canais desacoplados só é válido para a linearização padrão da haste. Conforme será visto mais adiante, a realimentação exata da haste, que será utilizada para simulação nesse projeto, mantém todos os canais acoplados. 


\section{Capítulo 4}

\section{Controle}

Após definido e analisado o modelo matemático do sistema a ser controlado, inicia-se o projeto do sistema de controle. Neste capítulo, um controlador para o sistema é desenvolvido e testado por de simulações dinâmicas.

\subsection{Definição da técnica de controle}

O princípio utilizado para o projeto do sistema de controle se baseia nas seguintes idéias:

\section{Haste}

Primeiramente, será considerada apenas a dinâmica da haste, estabilizando o pêndulo e abandonando a intenção de controlar as rodas de reação e os motores elétricos.

Será projetada uma realimentação linearizante $R L_{h}$ usando a teoria de linearização exata da dinâmica da haste, conforme a Figura 4.1.

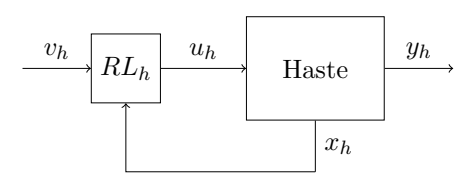

Figura 4.1: Diagrama de blocos do sistema composto pela haste com realimentação linearizante

Para que o sistema de controle seja robusto e não desperdice energia, este projeto será feito de modo que a linearização exata em malha fechada coincida com a linearização padrão do sistema (aproximação clássica por Jacobianos realizada na Seção 3.2.1).

Além da realimentação linearizante, também será projetada uma realimentação de estados $K_{h}$ com o objetivo de estabilizar o sistema da haste perfeitamente linearizado, conforme a Figura 4.2. 


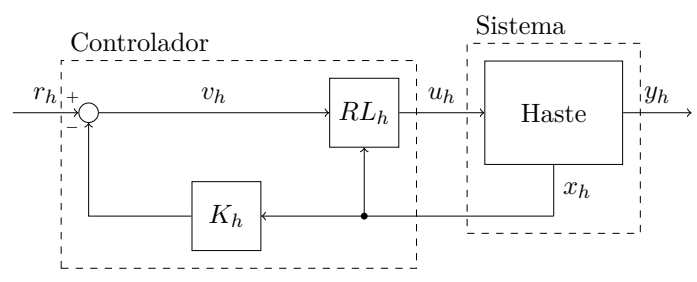

Figura 4.2: Diagrama de blocos do sistema composto pela haste com realimentação linearizante e realimentação de estados

Para o projeto da realimentação de estados $K_{h}$, o sistema com linearização exata será assumido igual ao sistema com linearização padrão, conforme a Figura 4.3.

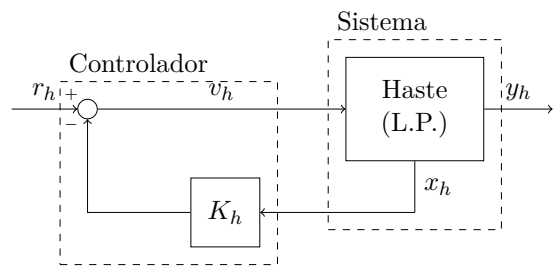

Figura 4.3: Diagrama de blocos do sistema composto pela haste com linearização padrão e realimentação de estados

Entretanto, para as simulações, o sistema da Figura 4.2 será utilizado.

Note que essa lei de controle será uma realimentação que envolve apenas os estados da haste, sem dependender dos estados das rodas de reação ou dos motores elétricos. O sistema em malha fechada obtido pode provocar velocidades das rodas de reação cada vez maiores.

\section{Haste com as rodas de reação}

Em seguida, a dinâmica das rodas de reação será incluída no sistema.

Será projetada uma realimentação de estados $K_{r}$ extra de pequenos ganhos para garantir a estabilidade do sistema completo. Como o coeficiente de atrito das rodas de reação é pequeno, em um primeiro momento eles serão assumidos como desprezíveis. Dessa forma, a entrada das rodas de reação e da haste torna-se a mesma, conforme a Figura 4.4. 


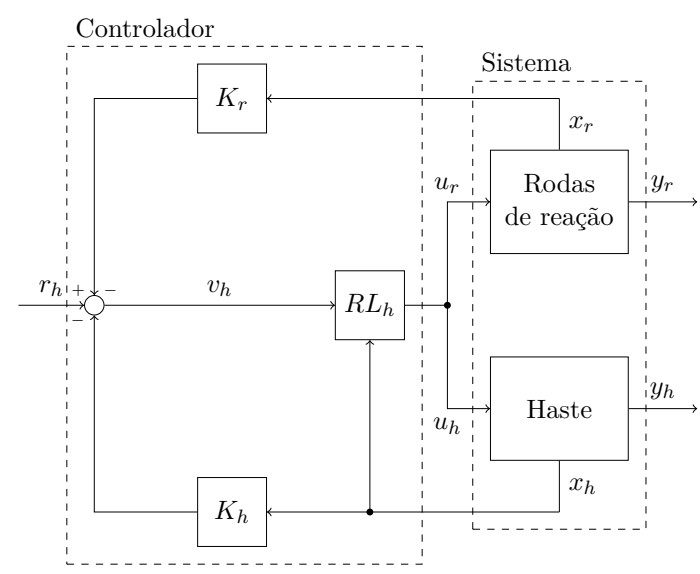

Figura 4.4: Diagrama de blocos do sistema composto pela haste e rodas de reação com realimentação linearizante, realimentação de estados da haste e realimentação de estados das rodas de reação desprezando o coeficiente de atrito das rodas

Note que, para $K_{r}$ nulo, a haste ficaria perfeitamente linearizada pela realimentação linearizante $R L_{h}$. No entanto, as rodas ficariam em malha aberta. A ideia então será projetar uma realimentação de estados $K_{r}$ tal que:

- $K_{r}$ seja pequeno o suficiente para que o comportamento da haste seja próximo ao do sistema perfeitamente linearizado

- $K_{r}$ garanta a estabilização do sistema como um todo

Novamente, o projeto da realimentação de estados $K_{r}$ será baseado no sistema com linearização padrão, conforme a Figura 4.5.

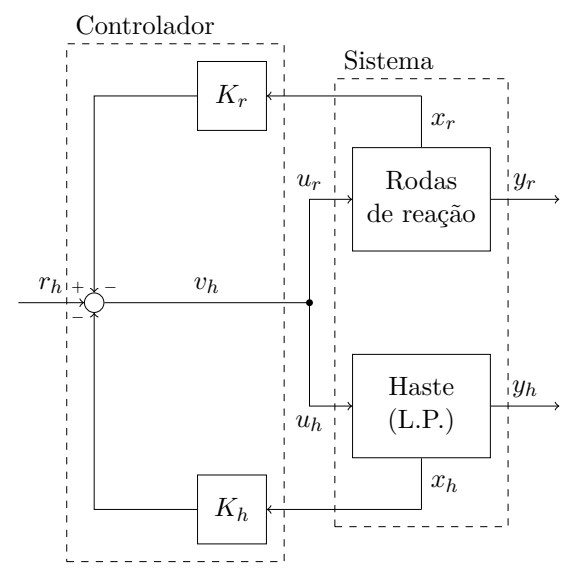

Figura 4.5: Diagrama de blocos do sistema composto pela haste com linearização padrão e rodas de reação com realimentação de estados da haste e realimentação de estados das rodas de reação desprezando o coeficiente de atrito das rodas

O projeto de $R L_{h}$ será realizado de tal forma que o sistema linearizado exatamente 
coincida com o sistema com linearização padrão, o garante que:

- A linearização de $R L_{h}$ é a identidade

- Não há esforços de controle desnecessários pois a lei de controle $R L_{h}$ se anula no ponto de equilíbrio

Entretanto, para as simulações será considerado o sistema com a linearização exata e sem desprezar os coeficientes de atrito das rodas de reação, conforme Figura 4.6.

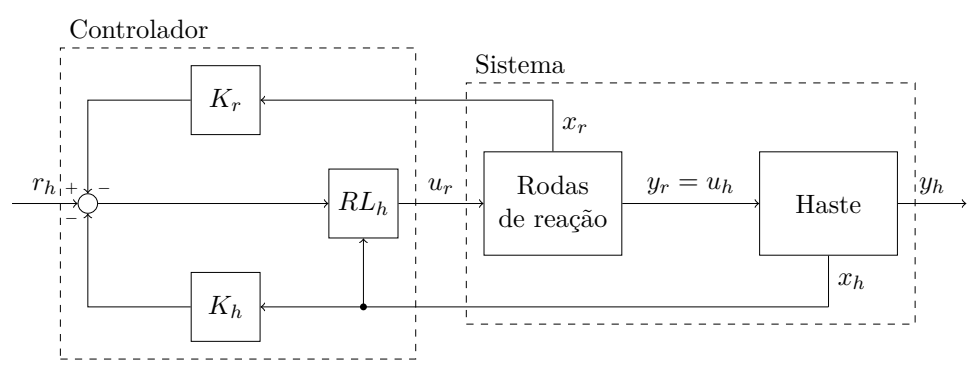

Figura 4.6: Diagrama de blocos do sistema composto pela haste e rodas de reação com realimentação linearizante da haste, realimentação de estados da haste e realimentação de estados das rodas de reação

\section{Haste com as rodas de reação e motores elétricos}

Por fim, a dinâmica dos motores elétricos também será incluída no sistema.

Pela dinâmica dos motores ser extremamente rápida se comparada com a dinâmica da haste e das rodas de reação, eles serão assumidos como sendo transdutores de torque de ganho constante $G_{m}$ e a realimentação dos estados das rodas de reação será desprezada. Dessa forma, deverá ser incorporado ao controlador um compensador de ganhos do motor, conforme a Figura 4.7.

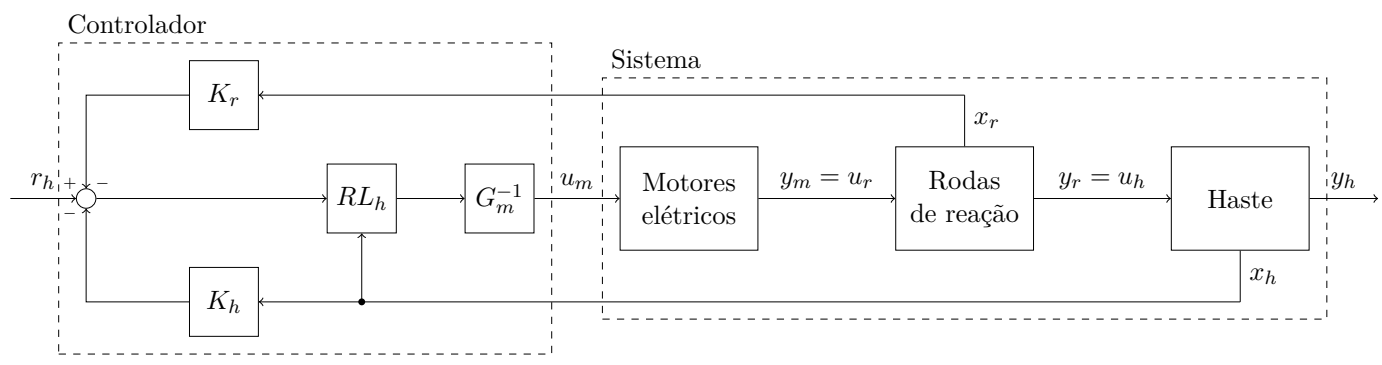

Figura 4.7: Diagrama de blocos do sistema composto pela haste, rodas de reação e motores elétricos com realimentação linearizante da haste, realimentação de estados da haste, realimentação de estados das rodas de reação e compensador de ganhos dos motores elétricos desprezando a realimentação dos estados das rodas nos motores

Entretanto, para as simulações não será desprezada a realimentação dos estados das rodas de reação nos motores elétricos, conforme Figura 4.8. 


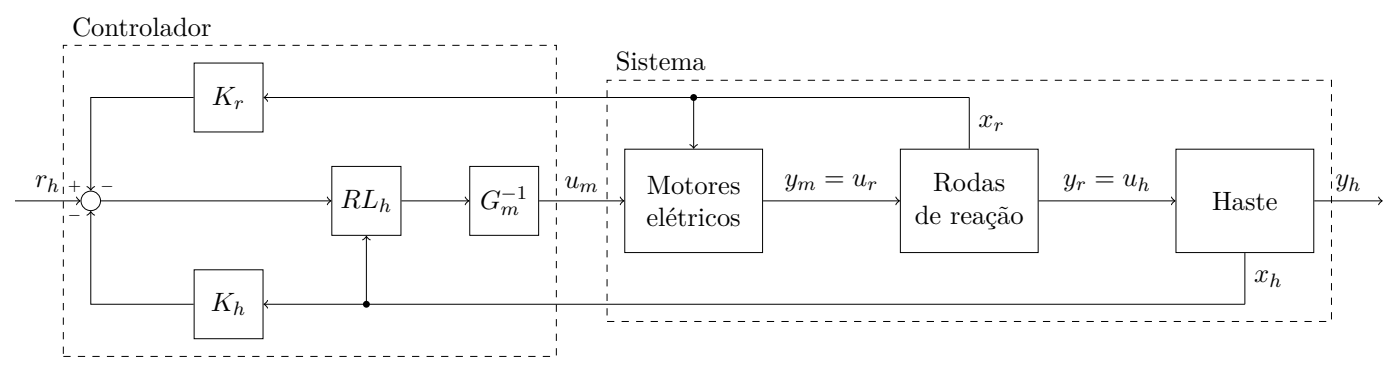

Figura 4.8: Diagrama de blocos do sistema composto pela haste, rodas de reação e motores elétricos com realimentação linearizante da haste, realimentação de estados da haste, realimentação de estados das rodas de reação e compensador de ganhos dos motores elétricos

\subsection{Controle da haste}

Conforme deduzido na Seção 3.2.1, o sistema não-linear que representa a dinâmica da haste pode ser representado da seguinte forma:

$$
\left\{\begin{array}{l}
\dot{x}_{h}=f_{h}\left(x_{h}\right)+g_{h}\left(x_{h}\right) u_{h} \\
y_{h}=h\left(x_{h}\right)
\end{array}\right.
$$

Será projetada uma realimentação linearizante usando a teoria da linearização exata.

\subsubsection{Realimentação linearizante}

A técnica de linearização exata consiste em encontrar uma realimentação de estados não-linear que permita o cancelamento exato das não linearidades do sistema. Dessa forma, o sistema em malha fechada torna-se linear, pelo menos quando ele for descrito em um novo sistema de coordenadas adequado.

O novo vetor de entradas das haste $v_{h}$ será dado por:

$$
v_{h}=\left[\begin{array}{l}
v_{x} \\
v_{y} \\
v_{z}
\end{array}\right]
$$

Como veremos adiante, a idéia do projeto do sistema de controle consiste em utilizar um controlador não linear (linearização exata) que promova a linearização perfeita do sistema. Para obter um controlador que não desperdice energia, optou-se por garantir que a linearização exata faça o sistema coincidir com o sistema obtido pela linearização padrão. A realimentação de estados que cumpre esses pré requisitos possui a forma:

$$
u_{h}=j_{h}\left(x_{h}\right)+k_{h}\left(x_{h}\right) v_{h}
$$

Onde: 


$$
j_{h}=P_{h}{ }^{-1}\left[N_{h}+O_{h}+M_{h} \widehat{A}_{h} x\right], \quad k_{h}=P_{h}{ }^{-1} M_{h} \widehat{B}_{h}
$$

O sistema com linearização exata pode ser representado conforme o diagrama de blocos da Figura 4.9.

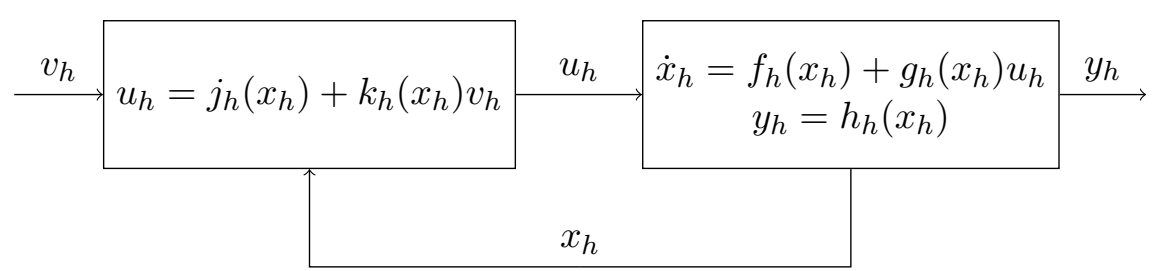

Figura 4.9: Diagrama de blocos do sub-sistema da haste com realimentação linearizante

Substituindo a Equação (4.3) na Equação (4.1), tem-se que:

$$
\left\{\begin{array}{l}
\dot{x}_{h}=f_{h}\left(x_{h}\right)+g_{h}\left(x_{h}\right) j_{h}\left(x_{h}\right)+g_{h}\left(x_{h}\right) k_{h}\left(x_{h}\right) v_{h} \\
y_{h}=h_{h}\left(x_{h}\right)
\end{array}\right.
$$

Subsituindo os valores de $f_{h}\left(x_{h}\right), g_{h}\left(x_{h}\right)$ e $h_{h}\left(x_{h}\right)$ definidos nas Equações (3.17) e os valores de $j_{h}\left(x_{h}\right)$ e $k_{h}\left(x_{h}\right)$ definidos nas Equações (4.4) na Equação (4.5), tem-se que:

$$
\left\{\begin{aligned}
\dot{x}_{h}=\left[\begin{array}{c}
\dot{q}_{h} \\
-M_{h}{ }^{-1}\left[N_{h}+O_{h}\right]
\end{array}\right]+\left[\begin{array}{c}
0 \\
M_{h}{ }^{-1} P_{h}
\end{array}\right]\left[P_{h}^{-1}\right. & {\left.\left[N_{h}+O_{h}+M_{h} \widehat{A}_{h} x_{h}\right]\right]+ } \\
& {\left[\begin{array}{c}
0 \\
M_{h}{ }^{-1} P_{h}
\end{array}\right]\left[P_{h}{ }^{-1} M_{h} \widehat{B}_{h}\right] v_{h} }
\end{aligned}\right.
$$

Simplificando:

$$
\left\{\begin{aligned}
\dot{x}_{h} & =\left[\begin{array}{c}
\dot{q}_{h} \\
\widehat{A}_{h} x_{h}
\end{array}\right]+\left[\begin{array}{c}
0 \\
\widehat{B}_{h} v_{h}
\end{array}\right] \\
y_{h} & =\left[q_{h}\right]
\end{aligned}\right.
$$

Como $x_{h}=\left[\begin{array}{ll}q_{h} & \dot{q}_{h}\end{array}\right]^{T}$, o sistema com linearização exata pode também ser representado da seguinte forma:

$$
\left\{\begin{array}{l}
\dot{x}_{h}=\left[\begin{array}{cc}
0 & I \\
\widehat{A}_{h}
\end{array}\right] x_{h}+\left[\begin{array}{c}
0 \\
\widehat{B}_{h}
\end{array}\right] v_{h} \\
y_{h}=\left[\begin{array}{ll}
\widehat{C}_{h} & 0
\end{array}\right] x_{h}
\end{array}\right.
$$

Ou de forma ainda mais simplificada como: 


$$
\left\{\begin{array}{l}
\dot{x}_{h}=A_{h} x_{h}+B_{h} v_{h} \\
y_{h}=C_{h} x_{h}
\end{array}\right.
$$

Onde:

$$
A_{h}=\left[\begin{array}{cc}
0 & I \\
\widehat{A}_{h}
\end{array}\right], \quad B_{h}=\left[\begin{array}{c}
0 \\
\widehat{B}_{h}
\end{array}\right], \quad C_{h}=\left[\begin{array}{ll}
\widehat{C}_{h} & 0
\end{array}\right]
$$

Dessa forma, o sistema com linearização exata passa a ser representado conforme o diagrama de blocos da Figura 4.10.

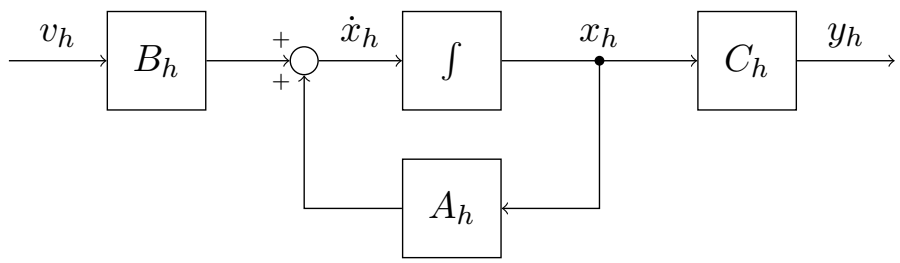

Figura 4.10: Diagrama de blocos do sub-sistema da haste linearizado (linearização exata)

Aqui fica evidente que o sistema em malha fechada com uma realimentação linearizante é um sistema linear. Além disso, comparando o diagrama de blocos do sistema com linearização exata da Figura 4.10 com o diagrama de blocos do sistema com linearização padrão da Figura 3.4, percebe-se que ambos são praticamente iguais. A única diferença é que enquanto a entrada do primeiro é $v_{h}$ a do segundo é $u_{h}$.

A realimentação de estados definida em na Equação (4.3) possui as seguintes propriedades:

$$
\begin{aligned}
& \left.\frac{\partial u_{h}\left(x_{h}, v_{h}\right)}{\partial x_{h}}\right|_{(0,0)}=0 \\
& \left.\frac{\partial u_{h}\left(x_{h}, v_{h}\right)}{\partial v_{h}}\right|_{(0,0)}=I
\end{aligned}
$$

Isso significa que os efeitos de $v_{h}$ e $u_{h}$ são muito similares quando o sistema está próximo da sua posição de equilíbrio, o que prova que o sistema com linearização padrão e o sistema com linearização exata obtidos são coincidentes. Dessa forma, para o projeto do sistema de controle, o sistema com linearização padrão será utilizado, enquanto que para as simulações dinâmicas, o sistema com linearização exata será utilizado.

\subsubsection{Realimentação de estados}

Um método relativamente simples de projetar um controlador para um sistema controlável em que todos os estados necessários estão acessíveis para medição é conhecido como realimentação de estados. É possível alocar os pólos de malha fechada do sistema 
em qualquer lugar que se desejar do plano complexo, ou seja, é possível especificar completamente o desempenho dinâmico sistema em malha fechada.

Infelizmente, o que pode ser obtido na teoria nem sempre pode ser obtido também na prática. Acelerar a resposta de um sistema naturalmente lento requer a utilização de grandes sinais de controle que um atuador pode não ser capaz de fornecer. A consequência geralmente é a saturação do atuador no maior sinal que ele pode fornecer. Em alguns casos esse comportamente é aceitável, porém em outros pode levar o sistema em malha fechada a ficar instável. Ou seja, não é possível alterar o comportamente dinâmico de malha aberta de forma muito drástica sem criar dificuldade práticas. (Friedland, 2005)

Um controlador de estados consiste em uma lei de controle em malha fechada onde a entrada do sistema $v_{h}$ é calculada em função de uma referência $r_{h}$ menos uma realimentação dos estados $x_{h}$ multiplicados por uma matriz de ganhos constantes $K_{h}$ :

$$
v_{h}=r_{h}-K_{h} x_{h}
$$

O sistema com realimentação de estados pode ser representado conforme o diagrama de blocos da Figura 4.11.

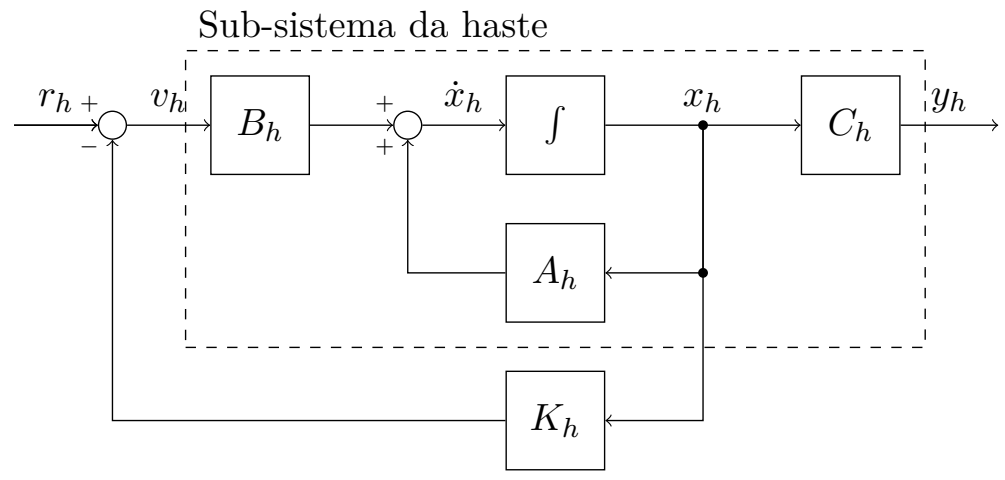

Figura 4.11: Diagrama de blocos do sub-sistema da haste linearizado (linearização exata) com realimentação de estados

Conforme visto anteriormente, o sistema em malha aberta é expresso da seguinte forma:

$$
\left\{\begin{array}{l}
\dot{x}_{h}=A_{h} x_{h}+B_{h} v_{h} \\
y_{h}=C_{h} x_{h}
\end{array}\right.
$$

Substituindo a Equação (4.13) na Equação (4.14) o sistema em malha fechada passa a ser expresso da seguinte forma:

$$
\left\{\begin{array}{l}
\dot{x}_{h}=\left(A_{h}-B_{h} K_{h}\right) x_{h}+B_{h} r_{h} \\
y_{h}=C_{h} x_{h}
\end{array}\right.
$$


Esse sistema é análogo ao anterior, ou seja, a matriz de estados do sistema em malha fechada pode ser expressa como:

$$
\bar{A}_{h}=A_{h}-B_{h} K_{h}
$$

Assim o sistema em malha fechada também pode ser representado conforme o seguinte diagrama de blocos da Figura 4.12.

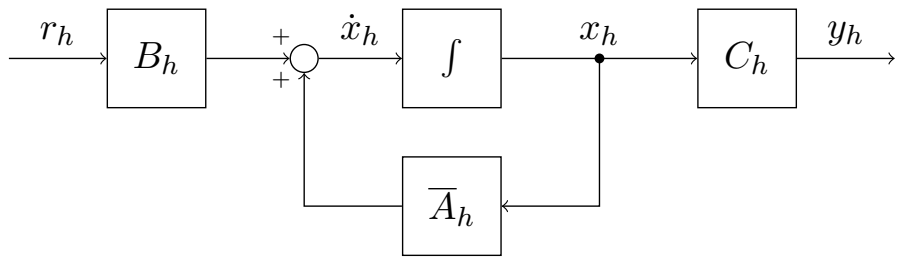

Figura 4.12: Diagrama de blocos do sub-sistema da haste linearizado (linearização exata) com realimentação de estados simplificado

O compotamente dinâmico do sistema em malha fechada (tempo de resposta, frequência natural, coeficiente de amortecimento, etc.) depende apenas de seus pólos, que por sua vez dependem apenas da matriz de estados $\bar{A}_{h}$. Como $\bar{A}_{h}=A_{h}-B_{h} K_{h}$, a matriz de ganhos $K_{h}$ deve ser escolhida de forma que o sistema em malha fechada tenha as propriedades desejadas. O mais simples é escolher os ganhos do controlador que alocam os pólos de malha fechada em posicões desejadas no plano complexo. Mas aí surge a seguinte questão: em que lugar do plano complexo os pólos de malha fechada devem ser alocados? Uma técnica bastante comum é conhecida como controle ótimo.

A primeira razão para se procurar projetar um controlador ótimo é que para um sistema com mais de uma entrada e/ou mais de uma saída a técnica de alocação de pólos não especifica completamente a matriz de ganhos. Por exemplo, um sistema de ordem $n$ com $m$ entradas e todos os estados acessíveis para realimentação tem $n \times m$ parâmetros da matriz de ganhos a serem determinados, mas apenas $n$ possíveis pólos de malha fechada a serem obtidos. Dado que há $m$ vezes mais parâmetros para serem especificados do que pólos, há infinitas soluções para se obter os mesmos pólos de malha fechada. Qual solução é a melhor? Qual algoritmo para determinação dos parâmetros é o melhor? Do posto de vista prático, é claro, a disponibilidade de mais parâmetros ajustáveis do que o menor número necessário para atingir a alocação dos pólos de malha fechada é uma grande vantagem para que outras coisas possam ser realizadas além de apenas alocar os pólos de malha fechada. Entretanto, a ausência de um algoritmo definitivo para determinar uma lei de controle única é um prejuízo para um projetista de sistemas que não sabe lidar com esse "empecilho" de riqueza de informações. Ao escolher uma lei de controle para otimizar o desempenho esse empecilho é evitado.

Uma razão mais convincente para utilizar um controlador ótimo é que o projetista pode não saber a posição desejada para os pólos em malha fechada. Selecionando posições longe da origem podem proporcionar um tempo de resposta rápido, porém requerer sinais de controle muito grandes para serem produzidos pelo atuador, que 
pode acabar saturando. Para evitar tais problemas muitas vezes é necessário limitar a velocidade de resposta ao que pode ser atingido sem saturação. (Friedland, 2005)

No projeto de um controlador ótimo, ao invés de se obter uma matriz de ganhos $K_{h}$ que garante os pólos de malha fechada desejados, é obtida uma matriz de ganhos $K_{h}$ que minimiza um critério de performance $J$ (ou "função custo") expressa como a integral de um termo quadrático dos estados do sistema $x_{h}$ mais um segundo termo quadrático das entradas do sistema $v_{h}$ :

$$
J=\int\left[x_{h}^{T}(\tau) Q(\tau) x_{h}(\tau)+v_{h}^{T}(\tau) R(\tau) v_{h}(\tau)\right] d \tau
$$

As matrizes $Q$ e $R$ da função custo são respectivamente a matriz de ponderação dos estados e a matriz de ponderação das saídas, que equilibram a importância relativa de erro dos estados $x_{h}$ e dos esforços de controle $v_{h}$. Ou seja, quanto maior for a matriz $Q$ em relação a $R$, mais prioridade será dada para minimizar os erros dos estados e menos prioridade será dada para minimizar os esforços de controle, enquanto que quanto maior for a matriz $R$ em relação a $Q$, mais prioridade será dada para minimizar os esforços de controle e menos importância será dada para minimizar os erros dos estados.

O fato de $Q$ e $R$ serem matrizes (e não constantes) ocorre pois dessa forma é possível dar uma prioridade específica para cada estado e entrada, e não necessáriamente a todos os estados e entradas como um todo. O caso mais simples é assumir $Q=C_{h}{ }^{T} C_{h}$, que da a mesma prioridade para todos os estados que são saídas do sistema $(\phi, \theta$ e $\psi)$, e $R=I$, que dá a mesma prioridade para todas as entradas do sistema $\left(v_{x}, v_{y}\right.$ e $\left.v_{z}\right)$. Ou seja:

$$
Q=\left[\begin{array}{llllll}
1 & 0 & 0 & 0 & 0 & 0 \\
0 & 1 & 0 & 0 & 0 & 0 \\
0 & 0 & 1 & 0 & 0 & 0 \\
0 & 0 & 0 & 0 & 0 & 0 \\
0 & 0 & 0 & 0 & 0 & 0 \\
0 & 0 & 0 & 0 & 0 & 0
\end{array}\right], \quad R=\left[\begin{array}{lll}
1 & 0 & 0 \\
0 & 1 & 0 \\
0 & 0 & 1
\end{array}\right]
$$

A matriz de ganhos $K_{h}$ que minimiza o critério de performance $J$ com $Q$ e $R$ determinados acima é ${ }^{1}$

$$
K_{h}=\left[\begin{array}{cccccc}
-1,68 & 0 & 0 & -0,20 & 0 & 0 \\
0 & -1,68 & 0 & 0 & -0,20 & 0 \\
0 & 0 & -1,00 & 0 & 0 & -0,04
\end{array}\right]
$$

\subsubsection{Estabilidade}

Conforme foi visto, a matriz de estados do sistema em malha fechada $\bar{A}_{h}$ é dada por $A_{h}-B_{h} K_{h}$. Agora que foi calculado o valor de $K_{h}$, torna-se possível determinar os pólos do sistema em malha fechada e verificar a estabilidade do mesmo.

\footnotetext{
${ }^{1}$ Valores calculados com o auxílio do MATLAB
} 
A equação característica do sistema em malha fechada é dada por:

$$
\operatorname{det}\left[s I-\bar{A}_{h}\right]=0
$$

Portanto, os pólos do sistema em malha fechada são:

$$
\left\{\begin{array}{l}
s_{1}=-28,00+j 27,78 \\
s_{2}=-28,00+j 27,78 \\
s_{3}=s_{4}=-8,22+j 4,88 \\
s_{5}=s_{6}=-8,22-j 4,88
\end{array}\right.
$$

Esses pólos podem ser representados no plano complexo conforme a Figura 4.13.

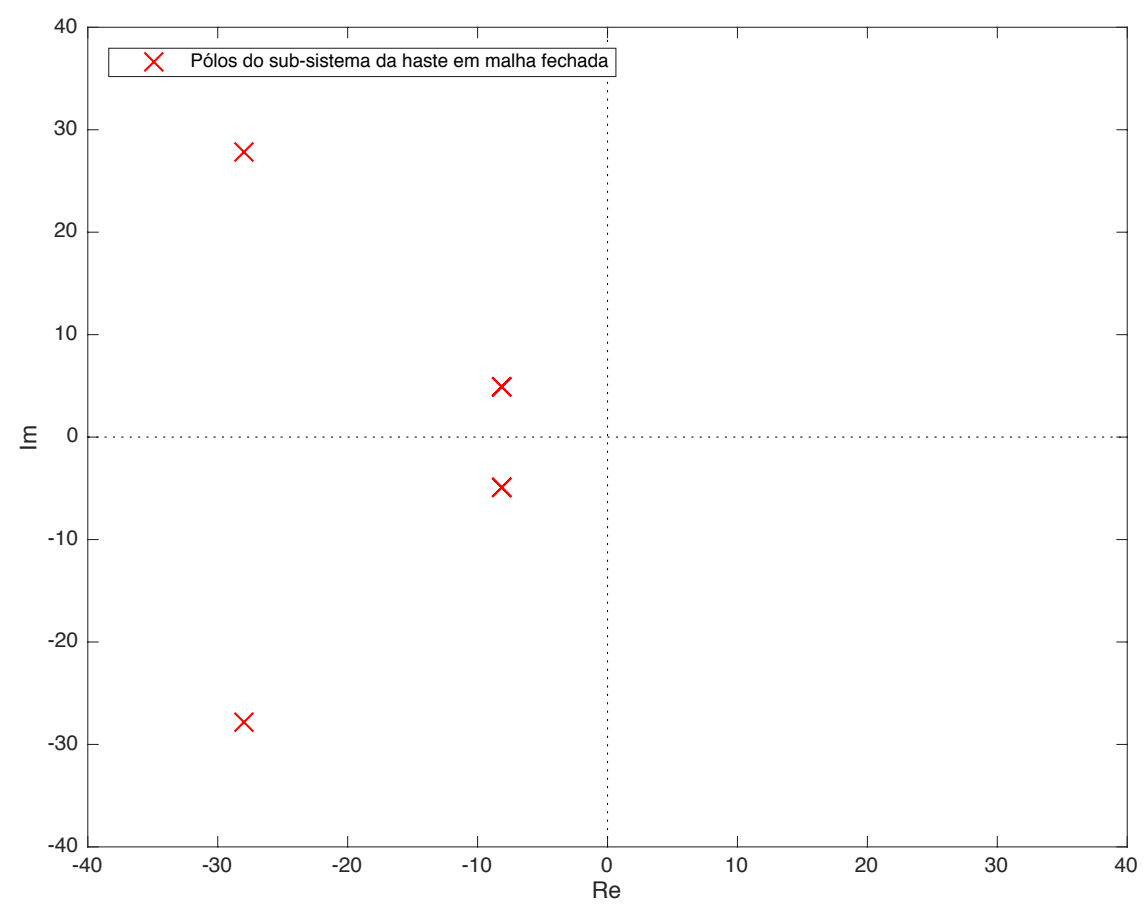

Figura 4.13: Pólos e zeros de transmissão do sistema com a haste em malha fechada

Observando a figura acima pode-se perceber que nenhum dos pólos está mais a direita do eixo imaginário, portanto é de se esperar que o sistema em malha fechada seja estável. Para verificar tal fato serão realizadas algumas simulações.

\subsubsection{Simulações}

\section{Simulação 1}

Supondo que a referência do sistema seja zero, ou seja, que $\phi_{r}, \theta_{r}$ e $\psi_{r}$ são nulos: 


$$
r_{h}=\left[\begin{array}{l}
0 \\
0 \\
0
\end{array}\right]
$$

E que o sistema encontra-se inicialmente com um pequeno deslocamento de sua posição de equilíbrio e sem velocidade, ou seja, que $\phi_{0}, \theta_{0}$ e $\psi_{0}$ são $5^{\circ}$ e que $\dot{\phi}, \dot{\theta}$ e $\dot{\psi}$ são nulos:

$$
x_{h 0}=\left[\begin{array}{c}
0,0873 \\
0,0873 \\
0,0873 \\
0 \\
0 \\
0
\end{array}\right]
$$

O resultado dessa simulação pode ser observado na Figura 4.14.
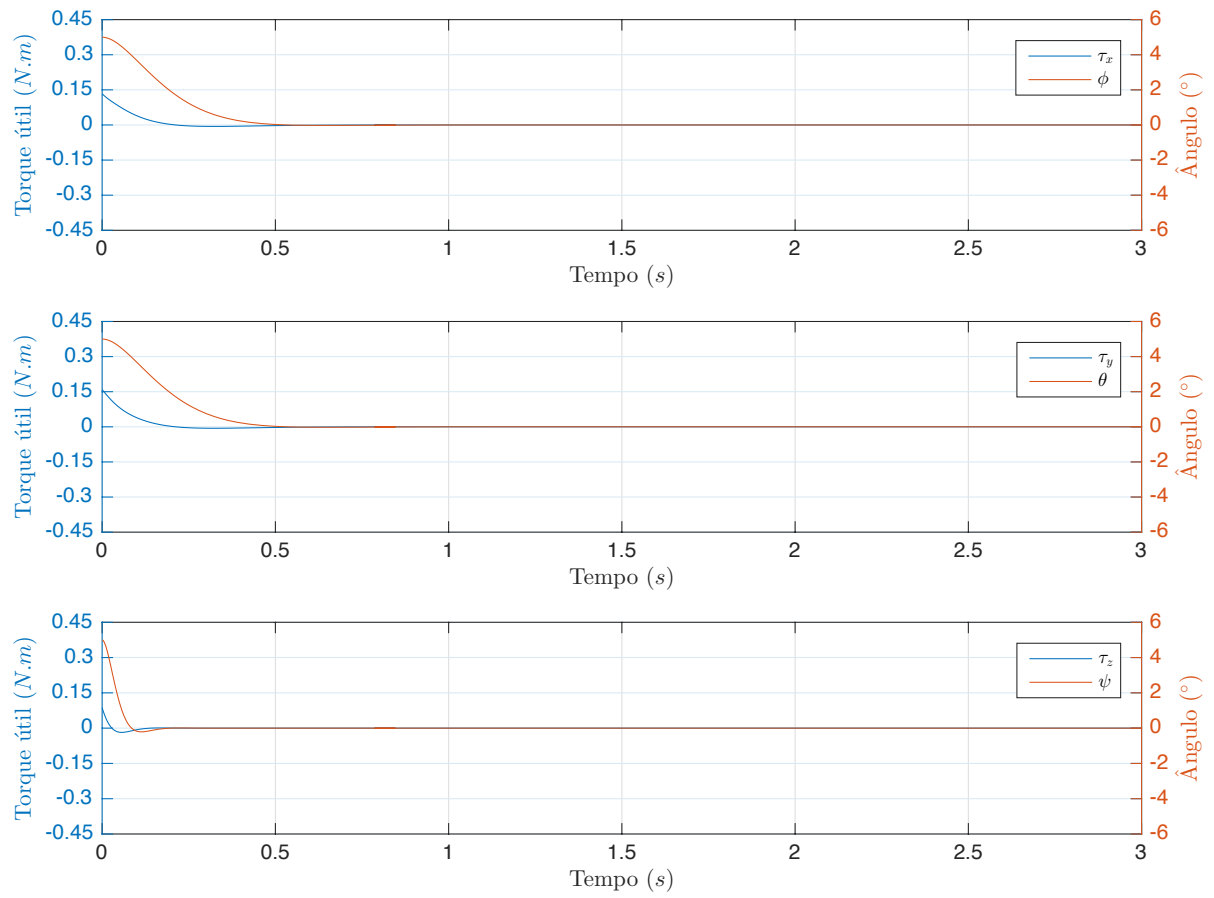

Figura 4.14: Resultados da simulação 1

Observando o resultado da simulação é trivial de se concluir que o sistema em malha fechada é estável dado que as saídas do sistema $\left(y_{h}\right)$ tendem aos sinais de referência do 
sistema em malha fechada $\left(r_{h}\right)$. Essa estabilidade é consequência dos pólos escolhidos, que estão todos à esquerda do eixo imaginário.

Para estabilizar os canais $\phi$ e $\theta$, o esforços de controle não ultrapassaram os $0,2 N . m$, o que não é um valor muito grande e, portanto, não deve apresentar problemas de saturação. É interessante de se notar também que os esforços de controle $v_{x}$ e $v_{y}$ não foram iguais, mesmo tendo visto que os canais desacoplados apresentam dinâmicas idênticas. Isso ocorreu pois as simulações estão sendo realizadas com o sistema nãolinear com uma realimentação linearizante, e nesse sistema não-linear os canais não estão desacoplados.

Já para estabilizar o canal $\psi$, o esforço de controle foi bem menor, não ultrapassando os $0,1 N$.m. Isso ocorre pois os canais $\phi$ e $\theta$ estão sujeitos a ação da gravidade enquanto que o canal $\psi$ não.

\section{Simulação 2}

O desenvolvimento de um protótipo físico envolve riscos associados a problemas construtivos que dizem respeito à simetria e precisão de contrução mecânica. Se o pêndulo não fosse simétrico ou os sensores dos ângulos $\phi, \theta$ e $\psi$ não estivessem perfeitamente alinhados com o pêndulo, a posição e orientação de equilíbrio do sistema não seria mais zero. Isso equivale a assumir que os estados medidos possuem um erro aditivo, conforme o diagrama de blocos da Figura 4.15.

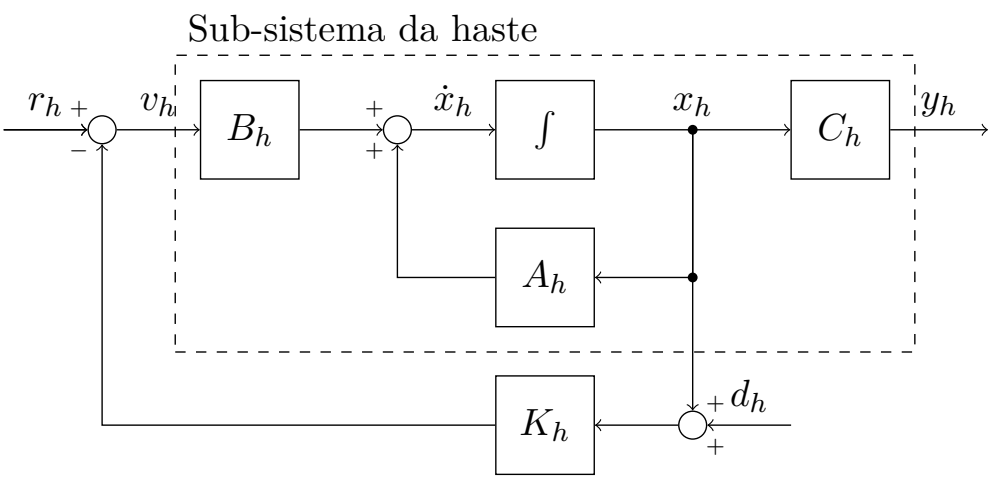

Figura 4.15: Diagrama de blocos do sub-sistema da haste linearizado (linearização exata) com realimentação de estados e erros de medição

Para entender exatamente o que aconteceria nesse caso, será feita uma nova simulacão igual a anterior, porém agora considerando um erro aditivo no sensor do ângulo $\phi$ de $2^{\circ}$ (por exemplo): 


$$
d_{h}=\left[\begin{array}{c}
0,0349 \\
0 \\
0 \\
0 \\
0 \\
0
\end{array}\right]
$$

O resultado dessa nova simulação pode ser observado na Figura 4.16.
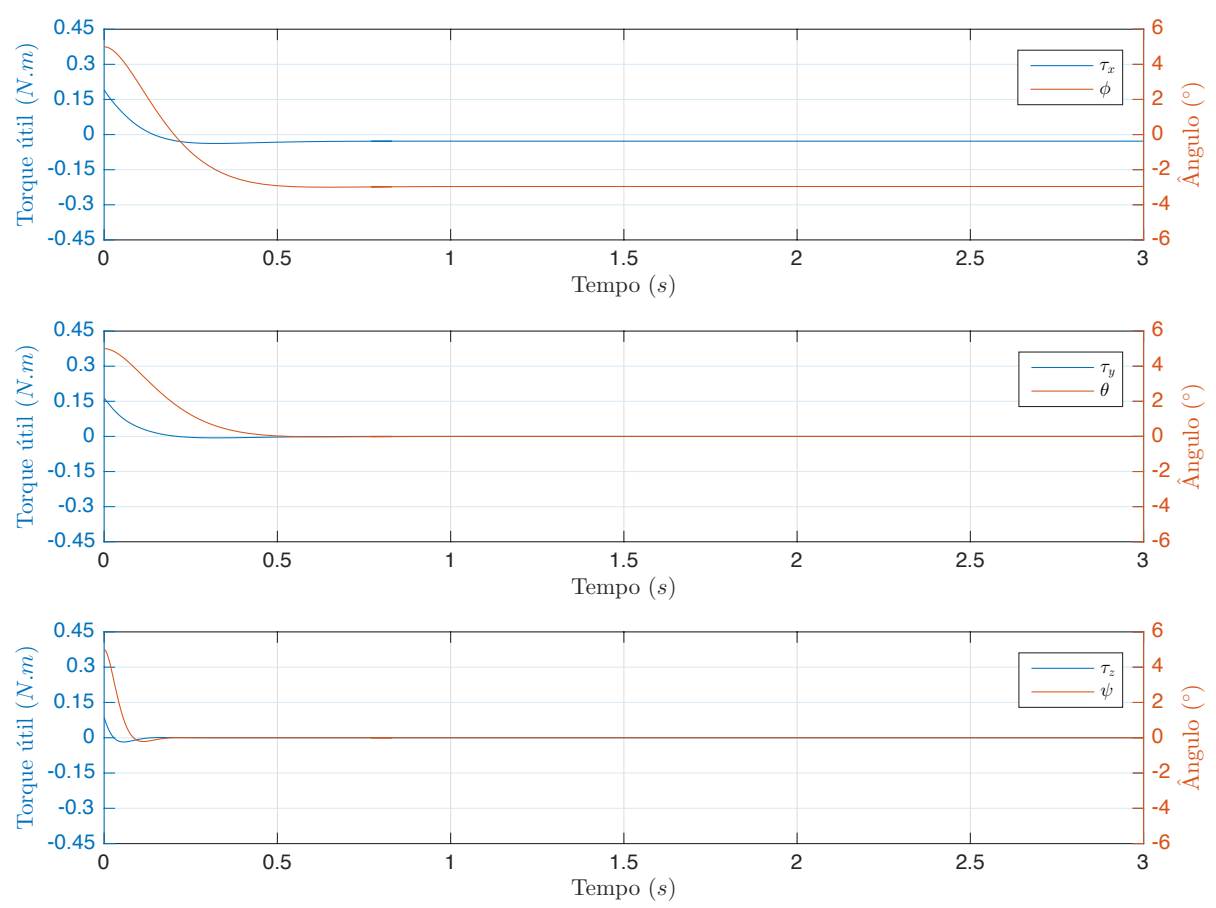

Figura 4.16: Resultados da simulação 2

Observando o resultado da simulação é interessante de se notar que apesar do ângulo $\phi$ possuir um erro de medição de $2^{\circ}$, o ângulo $\phi$ se estabilizou em torno de $-4^{\circ}$ ao invés de $-2^{\circ}$. Isso ocorre pois, como não há integradores no controlador, o erro em estado estacionário é diferente de zero. Além disso, para manter o ângulo $\phi$ em sua posição de equilíbrio, a entrada do sistema $\tau_{x}$ permaneceu constantemente diferente de zero. Em outras palavras, a roda de reação responsável pelo controle do canal $\phi$ ficou acelerando sem parar para manter o sistema em equilíbrio, pois o que o controlador "acha" que é a posição de equilíbrio na verdade não é.

Para resolver esse problema torna-se necessário levar em consideração a dinâmica das rodas de reação no modelo. A simples realimentação da velocidade das rodas de reação 
já resolveria esses dois problemas e as razões pelas quais isso ocorre serão explicadas na próxima seção.

\subsection{Controle da haste com as rodas de reação}

A matriz de ganhos $K_{h}$ obtida pelo método LQR é uma matriz diagonal. Isso significa que o controlador também possui canais desacoplados. Assumindo que:

$$
K_{h}=\left[\begin{array}{cccccc}
-k_{\phi} & 0 & 0 & -k_{\dot{\phi}} & 0 & 0 \\
0 & -k_{\theta} & 0 & 0 & -k_{\dot{\theta}} & 0 \\
0 & 0 & -k_{\psi} & 0 & 0 & -k_{\dot{\psi}}
\end{array}\right]
$$

O sistema com realimentação de estados ${ }^{2}$ pode ser representado de maneira desacoplada conforme o diagramas de blocos das Figura 4.17.

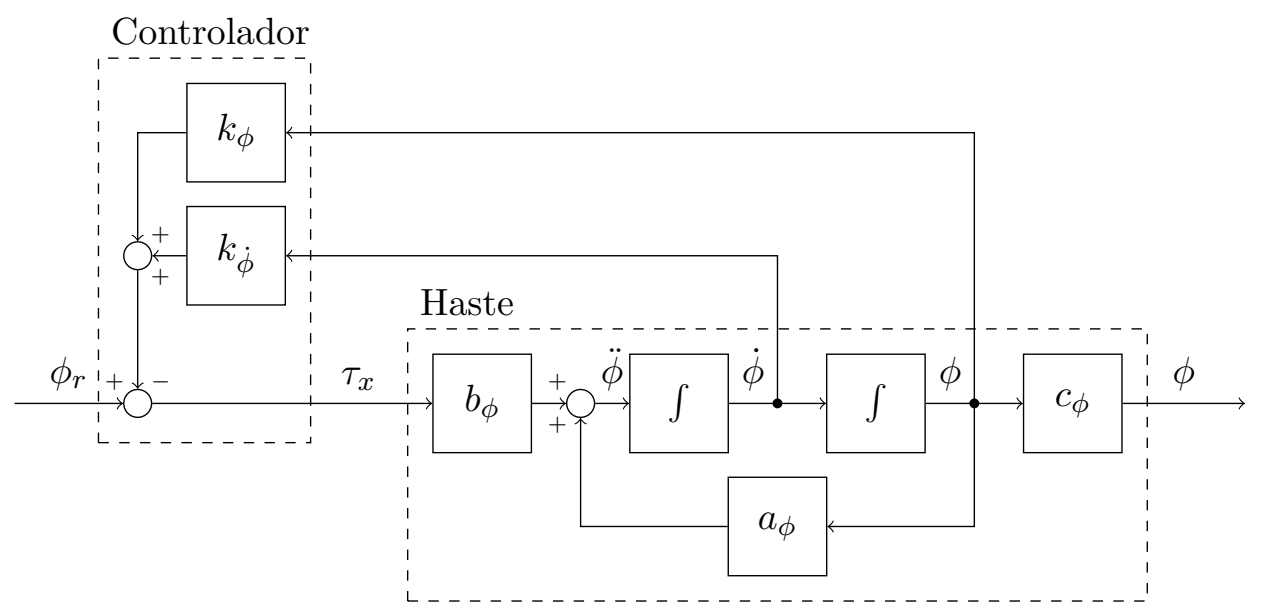

Figura 4.17: Diagrama de blocos do sistema considerado (haste) com realimentação de estados desacoplado

O sub-sistema do canal $\phi$ passa então a ser expresso da seguinte forma em malha fechada:

$$
\left\{\begin{array}{l}
\frac{d}{d t}\left[\begin{array}{c}
\phi \\
\dot{\phi}
\end{array}\right]=\left[\begin{array}{cc}
0 & 1 \\
a_{\phi}-b_{\phi} k_{\phi} & -b_{\phi} k_{\dot{\phi}}
\end{array}\right]\left[\begin{array}{c}
\phi \\
\dot{\phi}
\end{array}\right]+\left[\begin{array}{c}
0 \\
-b_{\phi}
\end{array}\right] \phi_{r} \\
y=\left[\begin{array}{ll}
c_{\phi} & 0
\end{array}\right]\left[\begin{array}{l}
\phi \\
\dot{\phi}
\end{array}\right]
\end{array}\right.
$$

Considerando agora a dinâmica das rodas de reação com uma realimentação adicional dos estados das rodas $(\dot{\alpha}, \dot{\beta}$ e $\dot{\gamma})$ multiplicados por um ganho $\left(k_{\dot{\alpha}}, k_{\dot{\beta}}\right.$ e $\left.k_{\dot{\gamma}}\right)$, o sistema passa a ser representado conforme o diagrama de blocos da Figura 4.18.

\footnotetext{
${ }^{2}$ Para simplificar só será representado o diagrama de blocos do canal $\phi$, mas o mesmo se aplica para os outros canais $\theta$ e $\psi$.
} 


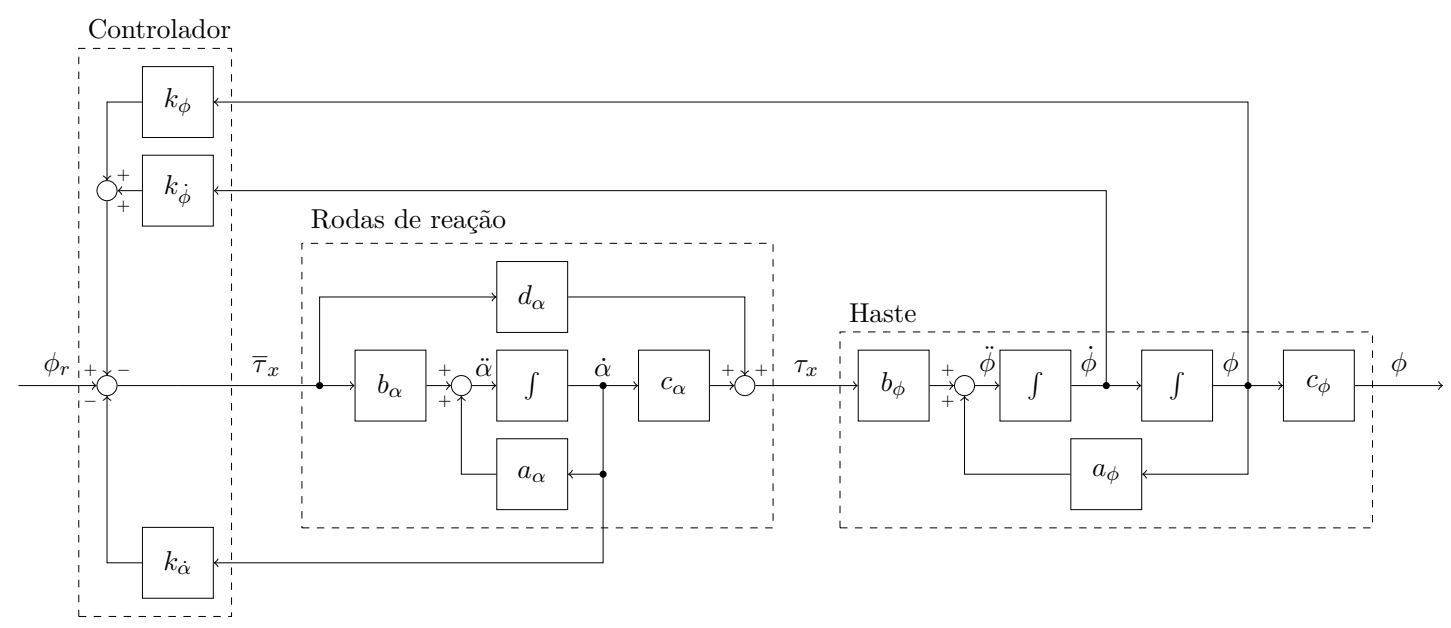

Figura 4.18: Diagrama de blocos do sistema considerado (haste e rodas de reação) com realimentação de estados desacoplado

Assumindo que o coeficiente de atrito das rodas de reação $b$ é muito pequeno, ele será desprezado para facilitar o projeto do controlador sem causar grandes impactos.

Como:

$$
a_{\alpha}=-\frac{b}{J_{r}}, \quad c_{\alpha}=-b,
$$

Para $b$ nulo tem-se que:

$$
a_{\alpha}=0, \quad c_{\alpha}=0
$$

Logo, o sistema da Figura 4.18 pode ser representado conforme o sistema da Figura 4.19 .

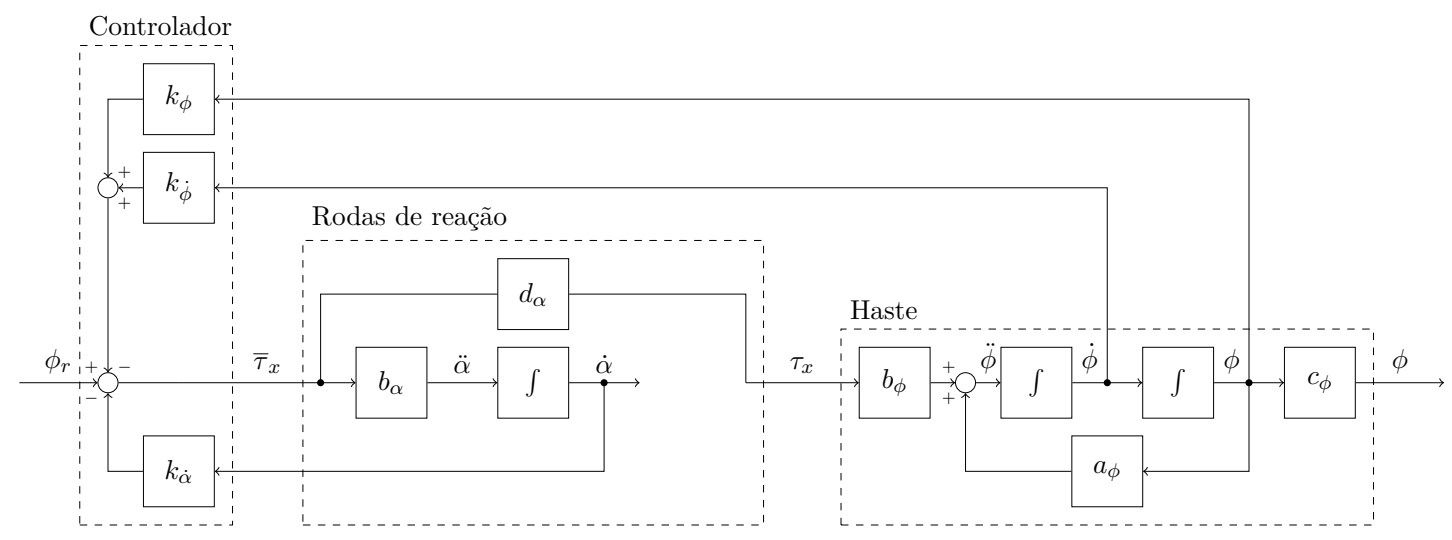

Figura 4.19: Diagrama de blocos do sistema considerado (haste e rodas de reação) com realimentação de estados desacoplado desprezando o coeficiente de atrito das rodas 
Sabendo também que:

$$
d_{\alpha}=1
$$

Logo, a entrada das rodas de reação e da haste torna-se a mesma:

$$
\bar{\tau}_{x}=\tau_{x}
$$

Dessa forma, o sub-sistema do canal $\phi$ agora passa a ser expresso da seguinte forma:

$$
\left\{\begin{array}{l}
\frac{d}{d t}\left[\begin{array}{c}
\phi \\
\dot{\phi} \\
\dot{\alpha}
\end{array}\right]=\left[\begin{array}{ccc}
0 & 1 & 0 \\
a_{\phi}-b_{\phi} k_{\phi} & -b_{\phi} k_{\dot{\phi}} & -b_{\phi} k_{\dot{\alpha}} \\
b_{\alpha} k_{\phi} & b_{\alpha} k_{\dot{\phi}} & b_{\alpha} k_{\dot{\alpha}}
\end{array}\right]\left[\begin{array}{c}
\phi \\
\dot{\phi} \\
\dot{\alpha}
\end{array}\right]+\left[\begin{array}{c}
0 \\
-b_{\phi} \\
b_{\alpha}
\end{array}\right] \phi_{r} \\
y=\left[\begin{array}{lll}
c_{\phi} & 0 & 0
\end{array}\right]\left[\begin{array}{c}
\phi \\
\dot{\phi} \\
\dot{\alpha}
\end{array}\right]
\end{array}\right.
$$

Uma maneira de determinar $k_{\dot{\alpha}}, k_{\dot{\beta}}$ e $k_{\dot{\gamma}}$ seria aplicando novamente o método LQR, porém agora para o sistema completo (considerando a dinâmica das rodas de reação), e obtendo assim uma nova matriz de ganhos $K_{r h}$ onde:

$$
K_{r h}=\left[\begin{array}{ccccccccc}
-k_{\phi} & 0 & 0 & -k_{\dot{\phi}} & 0 & 0 & k_{\dot{\alpha}} & 0 & 0 \\
0 & -k_{\theta} & 0 & 0 & -k_{\dot{\theta}} & 0 & 0 & k_{\dot{\beta}} & 0 \\
0 & 0 & -k_{\psi} & 0 & 0 & -k_{\dot{\psi}} & 0 & 0 & k_{\dot{\gamma}}
\end{array}\right]
$$

Entretanto, o sistema a ser simulado será o sistema não-linear, onde, diferentemente da Figura 4.19, os canais não estão desacoplados. Isso poderia gerar ganhos de realimentação das rodas demasiadamente elevados que alterassem de forma significativa a realimentação linearizante da haste. Além disso, poderia acelerar muito a dinâmica da haste e rodas de reação em malha fechada de modo que a dinâmica dos motores elétricos deixassem se ser desprezíveis e portanto eles não pudessem mais ser assumidos como transdutores de torque constante.

Dessa forma, serão mantidos os ganhos $k_{\phi}, k_{\dot{\phi}}, k_{\theta}, k_{\dot{\theta}}, k_{\psi}$ e $k_{\dot{\psi}}$ previamente calculados e os ganhos $k_{\dot{\alpha}}, k_{\dot{\beta}}$ e $k_{\dot{\gamma}}$ serão determinado de modo que eles sejam pequenos suficientes para que o comportamento da haste seja próximo ao perfeitamente linearizado mas que ao mesmo tempo garantam a estabilização do sistema como um todo.

\subsubsection{Critério de estabilidade de Routh}

Conforme visto na Seção 4.2.3, o eixo imaginário do plano complexo separa a região de estabilidade da região de instabilidade de um sistema. Se todos os pólos encontram-se no semi-plano esquerdo o sistema é estável, caso contrário o sistema é instável. Entretanto, existem alguns métodos mais simples para calcular a estabilidade de um sistema que não envolvem o cálculo dos pólos de um sistema. A mais antiga contribuição para este problema foi feita pelo E. J. Routh, que desenvolveu um algoritmo tabular simples através do qual é possível determinar se um determinado polinômio tem todas as suas 
raízes no semi-plano esquerdo do plano complexo sem efetivamente calcular as raízes desse polinômio.

O polinómio característico de um sistema de ordem $n$ a ser testado para a estabilidade é assumido como sendo da forma:

$$
s^{n}+a_{1} s^{n-1}+\ldots+a_{n-1} s+a_{n}
$$

A tabela de Routh correspondente a esse polinômio é construída conforme se mostra na Tabela 4.1. As duas primeiras linhas são obtidas por transcrição dos coeficientes do polinômio em linhas alternadas, cada linha suscetiva da tabela é completada utilizando valores das duas linhas anteriores, até que não haja mais termos a serem computados. $\mathrm{Na}$ margem esquerda há uma coluna com exatamente $n$ números $\delta_{1}, \delta_{2}, \ldots, \delta_{n}$. O teorema do algoritmo de Routh diz que as raízes desse polinômio encontram-se no semi-plano esquerdo, excluindo o eixo imaginário, se e apenas se todos os $\delta_{i}$ forem estritamente positivos.

Tabela 4.1: Tabela de Routh

$$
\begin{array}{c|ccccc} 
& 1 & a_{2} & a_{4} & a_{6} & \ldots \\
& a_{1} & a_{3} & a_{5} & a_{7} & \ldots \\
\hline \delta_{1}=1 / a_{1} & b_{1}=a_{2}-\delta_{1} a_{1} & b_{2}=a_{4}-\delta_{1} a_{5} & b_{3}=a_{6}-\delta_{1} a_{7} & \ldots & \\
\delta_{2}=a_{1} / b_{1} & c_{1}=a_{3}-\delta_{2} b_{2} & c_{2}=a_{5}-\delta_{2} b_{3} & \ldots & & \\
\delta_{3}=b_{1} / c_{1} & d_{1}=b_{2}-\delta_{3} c_{2} & \ldots & & & \\
\delta_{4}=c_{1} / d_{1} & \ldots & & & &
\end{array}
$$

Voltando ao sistema desse trabalho, a equação característca do sub-sistema do canal $\phi$ sistema é dada por:

$$
s^{3}+\left(b_{\phi} k_{\dot{\phi}}-b_{\alpha} k_{\dot{\alpha}}\right) s^{2}+\left(b_{\phi} k_{\phi}-a_{\phi}\right) s+a_{\phi} b_{\alpha} k_{\dot{\alpha}}
$$

Portanto, tem-se que:

$$
\left\{\begin{array}{l}
a_{1}=b_{\phi} k_{\dot{\phi}}-b_{\alpha} k_{\dot{\alpha}} \\
a_{2}=b_{\phi} k_{\phi}-a_{\phi} \\
a_{3}=a_{\phi} b_{\alpha} k_{\dot{\alpha}}
\end{array}\right.
$$

Aplicando a tabela de Routh chegamos nos seguintes valores de $\delta_{1}, \delta_{2}$ e $\delta_{3}$ : 


$$
\left\{\begin{array}{l}
\delta_{1}=-\frac{1}{b_{\alpha} k_{\dot{\alpha}}-b_{\phi} k_{\dot{\phi}}} \\
\delta_{2}=-\frac{b_{\alpha} k_{\dot{\alpha}}-b_{\phi} k_{\dot{\phi}}{ }^{2}}{b_{\phi}\left(a_{\phi} k_{\dot{\phi}}+b_{\alpha} k_{\dot{\alpha}} k_{\phi}-b_{\phi} k_{\phi} k_{\dot{\phi}}\right)} \\
\delta_{3}=\frac{b_{\phi}\left(a_{\phi} k_{\dot{\phi}}+b_{\alpha} k_{\dot{\alpha}} k_{\phi}-b_{\phi} k_{\phi} k_{\dot{\phi}}\right)}{a_{\phi} b_{\alpha} k_{\dot{\alpha}}\left(b_{\alpha} k_{\dot{\alpha}}-b_{\phi} k_{\dot{\phi}}\right)}
\end{array}\right.
$$

Para que $\delta_{1}, \delta_{2}$ e $\delta_{3}$ sejam positivos, $k_{\dot{\alpha}}$ precisa satisfazer as seguintes condições:

$$
\left\{\begin{array}{l}
k_{\dot{\alpha}} \leq \frac{b_{\phi} k_{\dot{\phi}}-1}{b_{\alpha}} \\
k_{\dot{\alpha}} \leq \frac{k_{\dot{\phi}}\left(a_{\phi}-b_{\phi} k_{\phi}\right)}{b_{\alpha} k_{\phi}}
\end{array}\right.
$$

Portanto, para cada canal tem-se as seguintes condições de estabilidade:

$$
\left\{\begin{array} { l } 
{ k _ { \dot { \alpha } } \leq 4 , 4 0 \times 1 0 ^ { - 4 } } \\
{ k _ { \dot { \alpha } } \leq 3 , 1 7 \times 1 0 ^ { - 4 } }
\end{array} \quad \left\{\begin{array} { l } 
{ k _ { \dot { \beta } } \leq 4 , 4 0 \times 1 0 ^ { - 4 } } \\
{ k _ { \dot { \beta } } \leq 3 , 1 7 \times 1 0 ^ { - 4 } }
\end{array} \quad \left\{\begin{array}{l}
k_{\dot{\gamma}} \leq 1,60 \times 10^{-3} \\
k_{\dot{\gamma}} \leq 1,60 \times 10^{-3}
\end{array}\right.\right.\right.
$$

Para que a realimentação das velocidades angulares das rodas de reação não deixe o sistema instável, os ganhos $k_{\dot{\alpha}}, k_{\dot{\beta}}$ e $k_{\dot{\gamma}}$ devem satisfazer as seguintes condições:

$$
\left\{\begin{array}{l}
k_{\dot{\alpha}} \leq 3,17 \times 10^{-4} \\
k_{\dot{\beta}} \leq 3,17 \times 10^{-4} \\
k_{\dot{\gamma}} \leq 1,60 \times 10^{-3}
\end{array}\right.
$$

Para certificar-se de que o sistema realmente se torna estável e determinar qual valores de $k_{\dot{\alpha}}, k_{\dot{\beta}}$ e $k_{\dot{\gamma}}$ dentro dessas condições serão adotadas, algumas simulações foram realizadas.

\subsubsection{Simulações}

\section{Simulação 3}

Inicialmente será adotado um valor para os ganhos equivalente a $110 \%$ do calculado como condição de estabilidade:

$$
\left\{\begin{array}{l}
k_{\dot{\alpha}}=3,48 \times 10^{-4} \\
k_{\dot{\beta}}=3,48 \times 10^{-4} \\
k_{\dot{\gamma}}=1,80 \times 10^{-3}
\end{array}\right.
$$

Retomando o mesmo cenário da última simulação, com referência nula, condição inicial com um pequeno deslocamento da posição e orientação de equilíbrio porém sem velocidade e um erro aditivo no canal $\phi$, o resultado da simulação pode ser observado na Figura 4.20. 

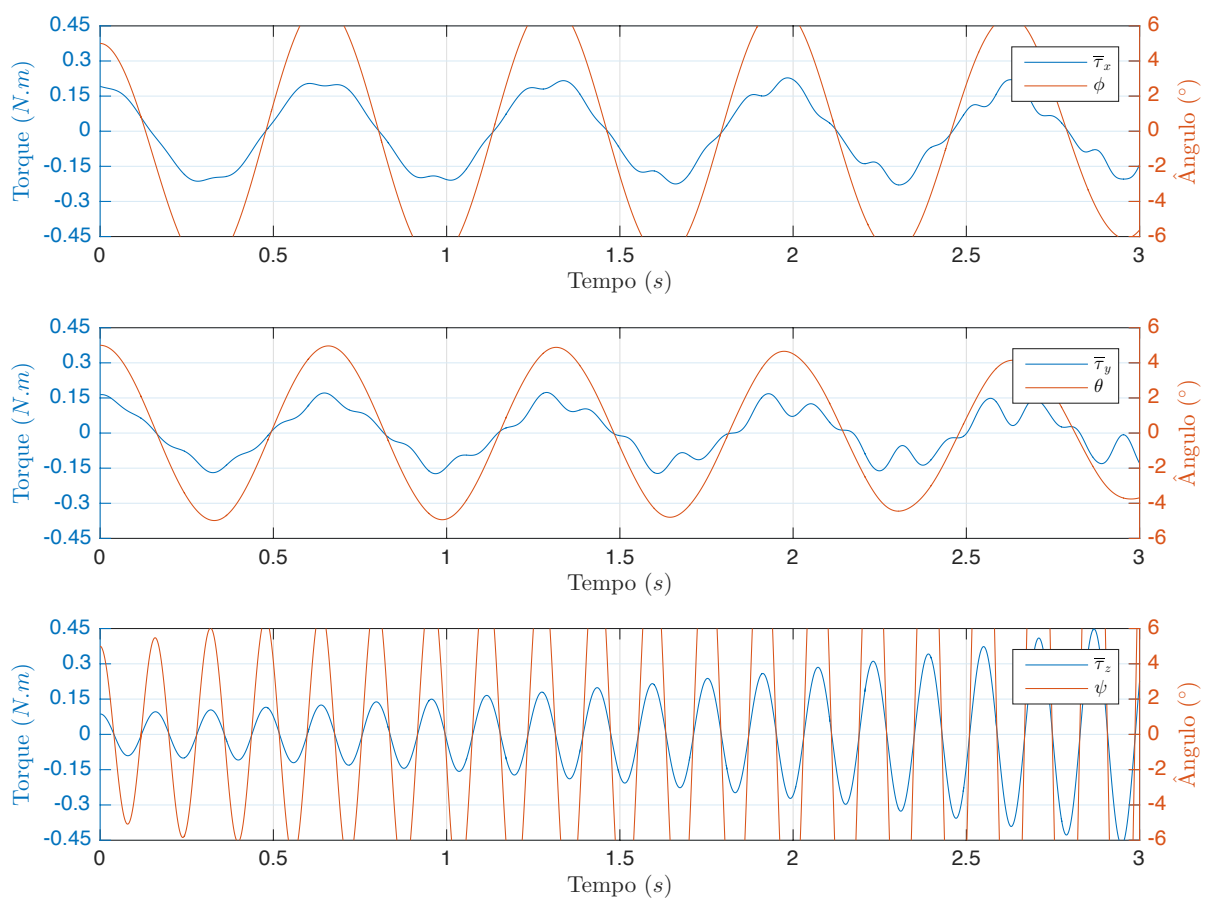

Figura 4.20: Resultado da simulação 3

Conforme pode ser observado na figura acima, a realimentação das rodas desestabilizou o sistema. Isso já era esperado pois as condições de estabilidade de Routh não foram satisfeitas (já que os valores adotados para $k_{\dot{\alpha}}, k_{\dot{\beta}}$ e $k_{\dot{\gamma}}$ foram $110 \%$ dos máximos permitidos).

\section{Simulação 4}

Adotadando agora um valor para os ganhos equivalente a $90 \%$ do calculado como condição de estabilidade:

$$
\left\{\begin{array}{l}
k_{\dot{\alpha}}=2,85 \times 10^{-4} \\
k_{\dot{\beta}}=2,85 \times 10^{-4} \\
k_{\dot{\gamma}}=1,40 \times 10^{-3}
\end{array}\right.
$$

O resultado da simulação pode ser observado na Figura 4.21. 

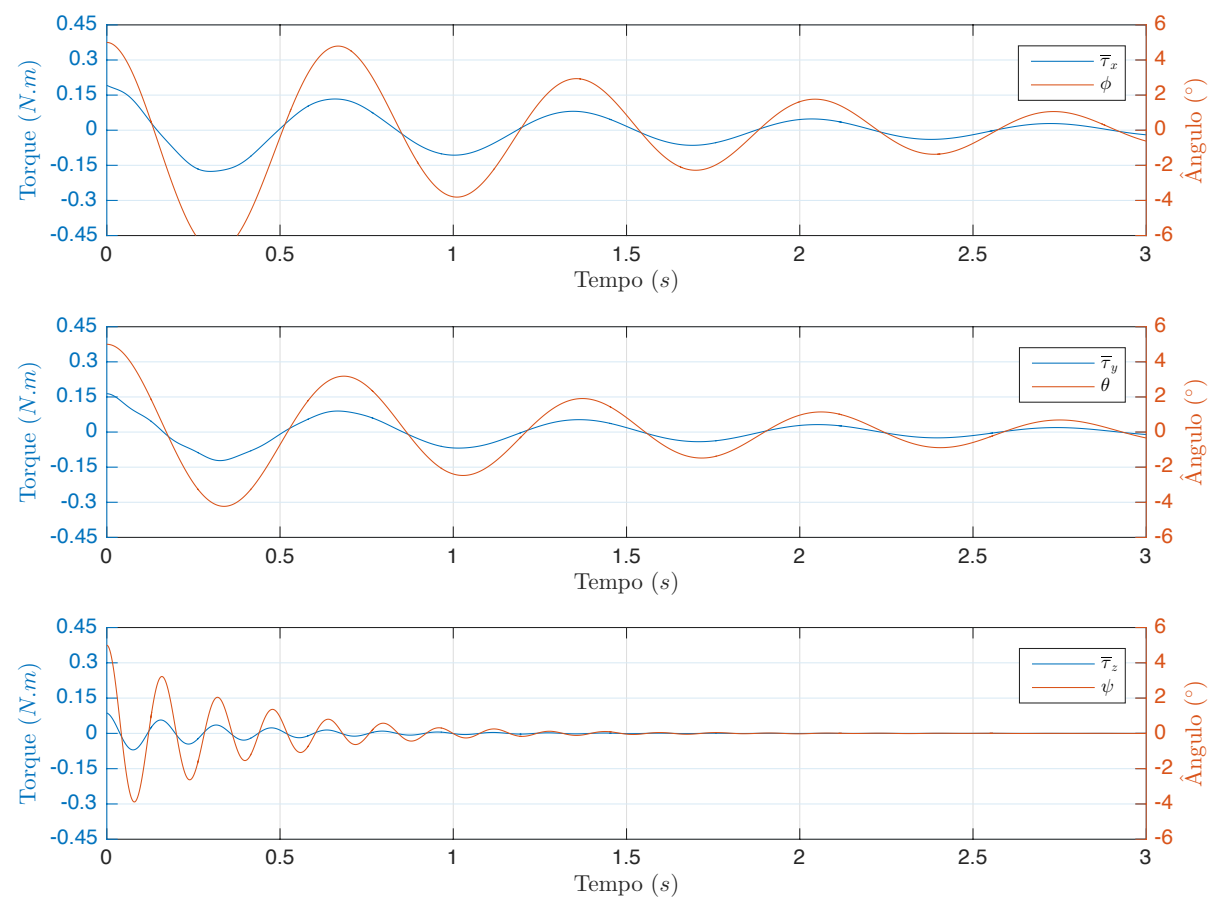

Figura 4.21: Resultado da simulação 4

Neste caso a realimentação das rodas manteve o sistema estável, mostrando que os critérios de estabilidade de Routh estão corretos, já que para valores um pouco acima do máximo permitido o sistema fica instável, enquanto que para valores um pouco abaixo do máximo permitido o sistema fica estável.

Entretanto, estabilidade não é o único objetivo de controle. Conforme pode ser observado na figura acima, o coeficiente de amortecimento do sistema ficou muito baixo. Portanto os valores de $k_{\dot{\alpha}}, k_{\dot{\beta}}$ e $k_{\dot{\gamma}}$ adotados ainda não são satisfatórios.

\section{Simulação 5}

Adotadando desta vez um valor para os ganhos equivalente a $50 \%$ do calculado como condição de estabilidade:

$$
\left\{\begin{array}{l}
k_{\dot{\alpha}}=1,58 \times 10^{-4} \\
k_{\dot{\beta}}=1,58 \times 10^{-4} \\
k_{\dot{\gamma}}=0,80 \times 10^{-3}
\end{array}\right.
$$

O resultado da simulação pode ser observado na Figura 4.22. 

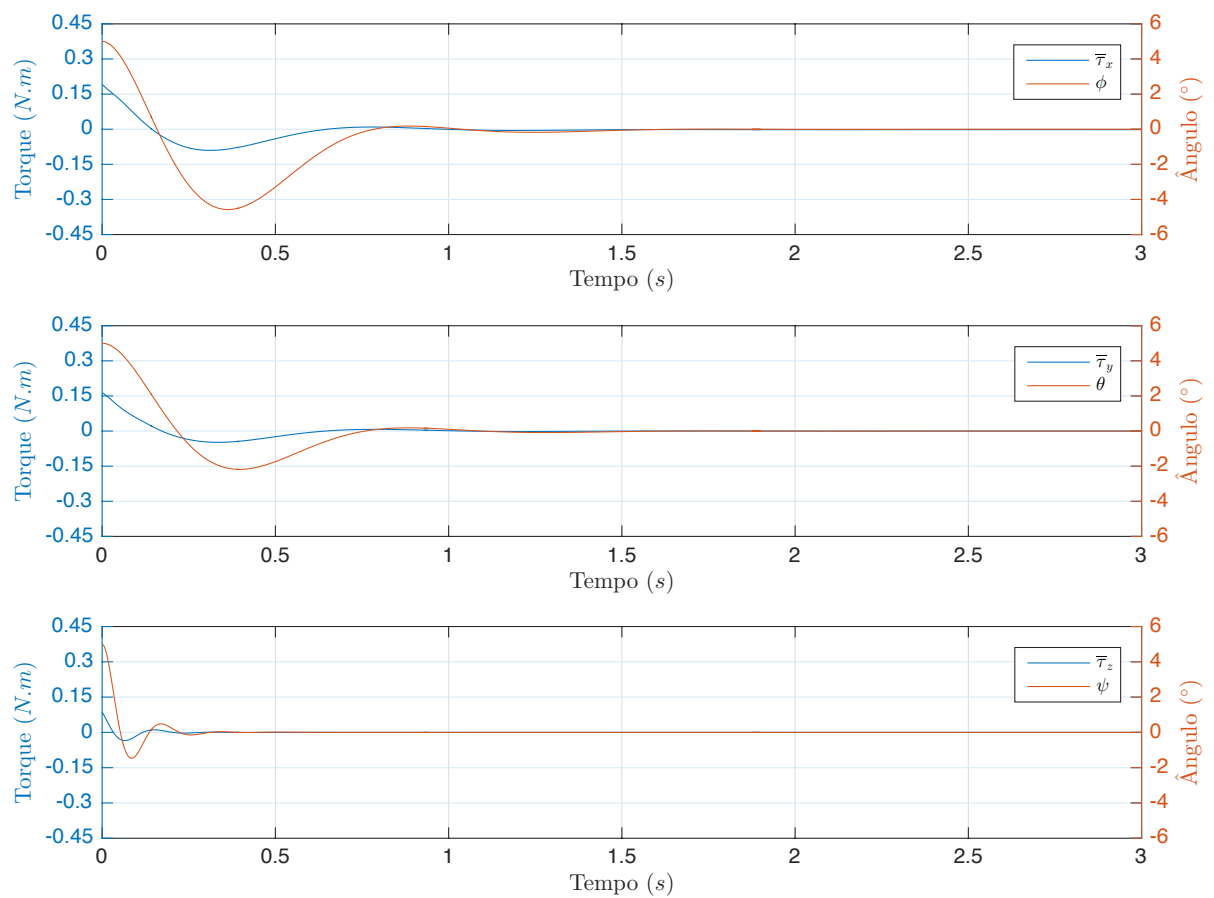

Figura 4.22: Resultado da simulação 5

Nesse caso a realimentação das rodas também manteve o sistema estável e o coeficiente de amortecimento do sistema não é mais tão baixo. Além disso, conforme pode ser observado na figura acim, a o problema dos erros de medição foi corrigido dado que o $\phi$ passou a se estabilizar na condição de equilíbrio $0^{\circ}$ e o esforço de controle $\tau_{x}$ tende a zero.

\section{Simulação 6}

Adotando um valor para os ganhos menor ainda, equivalente a $10 \%$ do calculado como condição de estabilidade:

$$
\left\{\begin{array}{l}
k_{\dot{\alpha}}=0,32 \times 10^{-4} \\
k_{\dot{\beta}}=0,32 \times 10^{-4} \\
k_{\dot{\gamma}}=0,16 \times 10^{-3}
\end{array}\right.
$$

O resultado da simulação pode ser observado na Figura 4.23. 

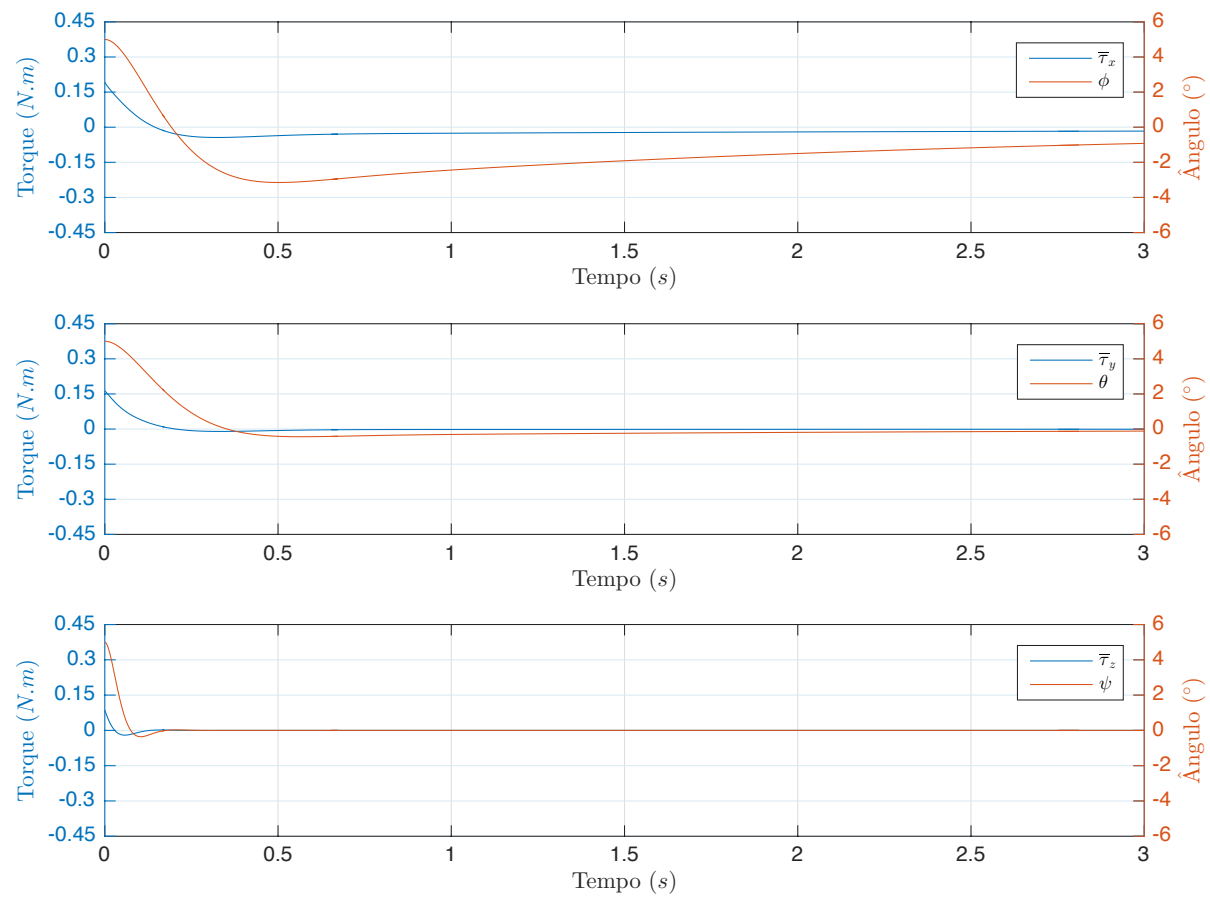

Figura 4.23: Resultado da simulação 6

Conforme pode ser observado acima, o sistema continua estável porém desta vez o problema foi o tempo de resposta. Isso já era esperado dado que quanto menor é o coeficiente de amortecimento maior é o tempo de resposta. Portanto, para determinação do ganho de realimentação das rodas de reação não basta apenas satisfazer as condições de estabilidade do critério de Routh como também é necessário realizar algumas simulações para se certificar de que o sistema possui o comportamento dinâmico desejado.

\subsection{Controle da haste com as rodas de reação e motores elétricos}

O último etapa de projeto do controlador é considerar a dinâmica dos motores elétricos no sistema, conforme a Figura 4.24. 


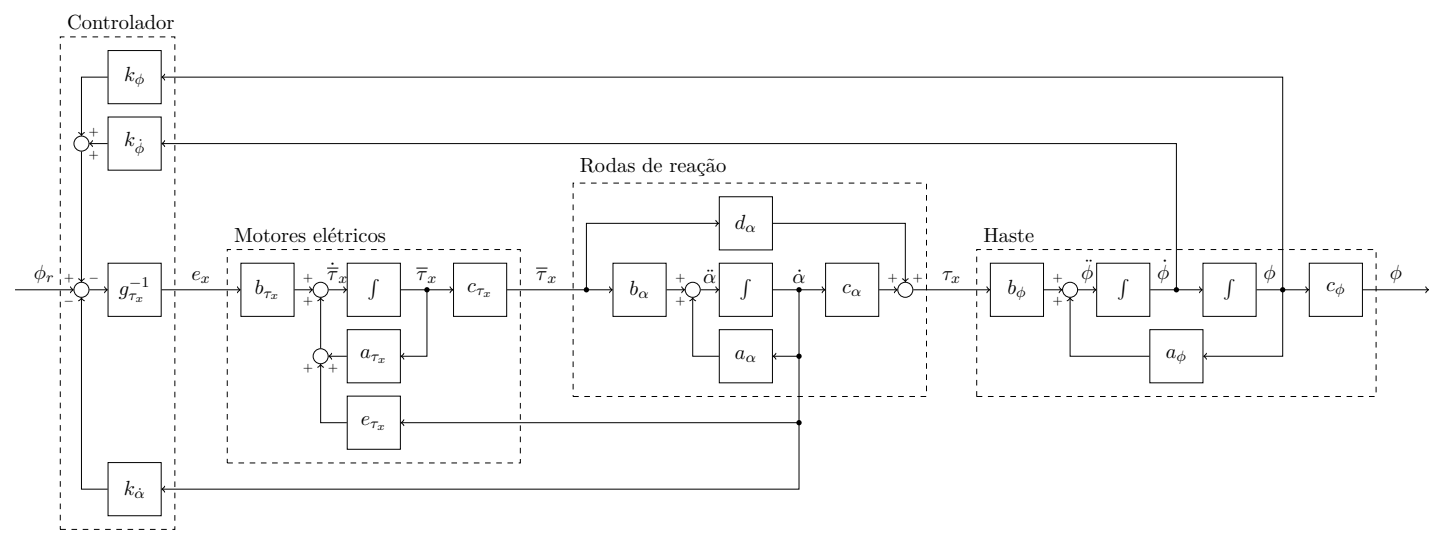

Figura 4.24: Diagrama de blocos do sistema considerado (haste, rodas de reação e motores elétricos) com realimentação de estados desacoplado

Pela dinâmica dos motores ser extremamente rápida se comparada com a dinâmica da haste e das rodas de reação, eles serão assumidos como sendo transdutores de torque de ganhos constantes $g_{\tau_{x}}, g_{\tau_{y}}$ e $g_{\tau_{z}}$ e um compensador de ganhos deverá ser incorprorado ao controlador.

\subsubsection{Compensador de ganhos}

Em um primeiro momento, a realimentação de estados das rodas de reação nos motores elétricos será desprezada. Dessa forma, o sistema da Figura 4.24 passa a ser representado conforme a Figura 4.25.

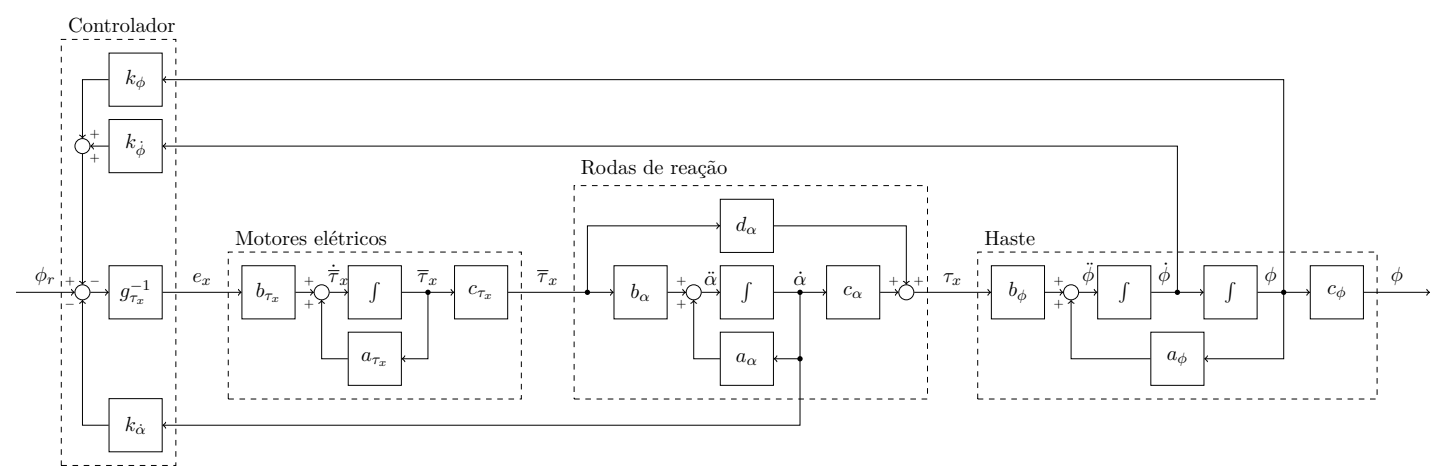

Figura 4.25: Diagrama de blocos do sistema considerado (haste, rodas de reação e motores elétricos) com realimentação de estados desacoplado desprezando a realimentação dos estados das rodas nos motores

O ganho constante dos motores elétricos é dado por:

$$
g_{\tau_{x}}=-\frac{c_{\tau_{x}} b_{\tau_{x}}}{a_{\tau_{x}}}
$$


Substituindo os valores de $a_{\tau_{x}}, b_{\tau_{x}}$ e $c_{\tau_{x}}$ definidos na Seção 2.5 , tem-se que:

$$
g_{\tau_{x}}=\frac{k_{\tau}}{R}
$$

Assim, para cada canal são obtidos os seguintes compensadores de ganhos constantes dos motores elétricos:

$$
g_{\tau_{x}}^{-1}=\frac{R}{k_{\tau}}, \quad g_{\tau_{y}}^{-1}=\frac{R}{k_{\tau}}, \quad g_{\tau_{z}}^{-1}=\frac{R}{k_{\tau}}
$$

Entretanto, assim como nas simulações anteriores, o sistema a ser simulado será o sistema não-linear, onde, diferentemente da Figura 4.25, os canais não estão desacoplados. Além disso, no sistema a ser simulado os motores elétricos possuem sua dinâmica própria (e não são ganhos constantes) e a realimentação dos estados das rodas de reação não é desprezada. Para garantir que mesmo com todas essas simplificações o projeto do controlador vai funcionar, torna-se necessário verificar o resultado das simulações.

\subsubsection{Simulações}

\section{Simulação 7}

Adotadando o valor dos ganhos $k_{\dot{\alpha}}, k_{\dot{\beta}}$ e $k_{\dot{\gamma}}$ da simulação 5 , que foi a que apresentou o melhor desempenho:

$$
\left\{\begin{array}{l}
k_{\dot{\alpha}}=1,58 \times 10^{-4} \\
k_{\dot{\beta}}=1,58 \times 10^{-4} \\
k_{\dot{\gamma}}=0,80 \times 10^{-3}
\end{array}\right.
$$

E o valor dos compensadores $g_{\tau_{x}}^{-1}, g_{\tau_{y}}^{-1}$ e $g_{\tau_{z}}^{-1}$ calculados acima:

$$
\left\{\begin{array}{l}
g_{\tau_{x}}^{-1}=20 \\
g_{\tau_{y}}^{-1}=20 \\
g_{\tau_{z}}^{-1}=20
\end{array}\right.
$$

O resultado da simulação pode ser observado na Figura 4.26. 

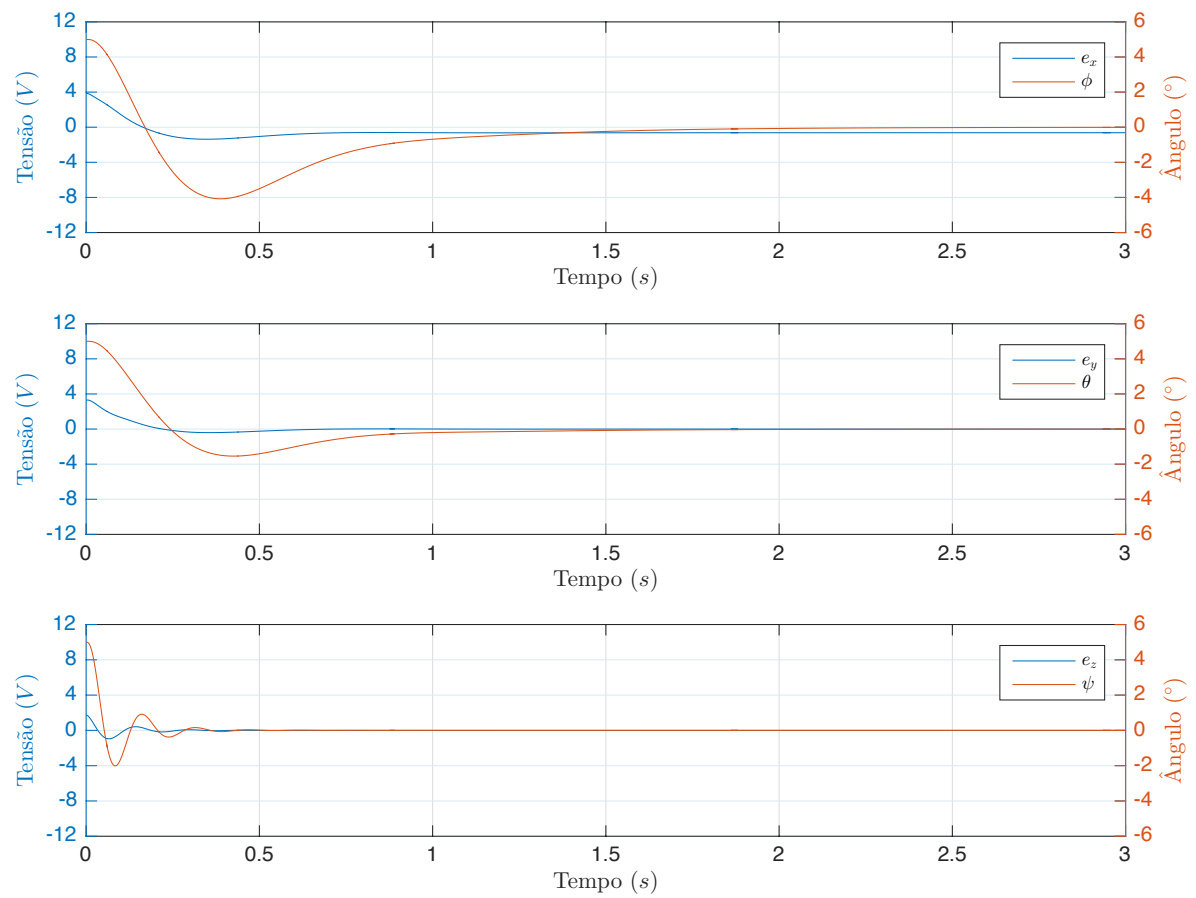

Figura 4.26: Resultado da simulação 7

Conforme pode ser observado, o sistema ficou um pouco mais lento do que o simulado anteriormente. Entretanto, os esforços de controle ficaram dentro de valores aceitáveis, não ultrapassando os $5 V$, e o pêndulo continua sendo estabilizado. Isso significa que a simplificação da dinâmica dos motores elétricos como transdutores de torques constantes para projeto do controlador foi aceitável.

\section{Simulação 8}

Para que a dinâmica do sistema volte a ficar um pouco mais rápida, serão adotados valores para os ganhos $k_{\dot{\alpha}}, k_{\dot{\beta}}$ e $k_{\dot{\gamma}}$ um pouco maiores, equivalentes a $70 \%$ dos calculados como condição de estabilidade:

$$
\left\{\begin{array}{l}
k_{\dot{\alpha}}=2,22 \times 10^{-4} \\
k_{\dot{\beta}}=2,22 \times 10^{-4} \\
k_{\dot{\gamma}}=1,10 \times 10^{-3}
\end{array}\right.
$$

O resultado da simulação pode ser observado na Figura 4.27. 

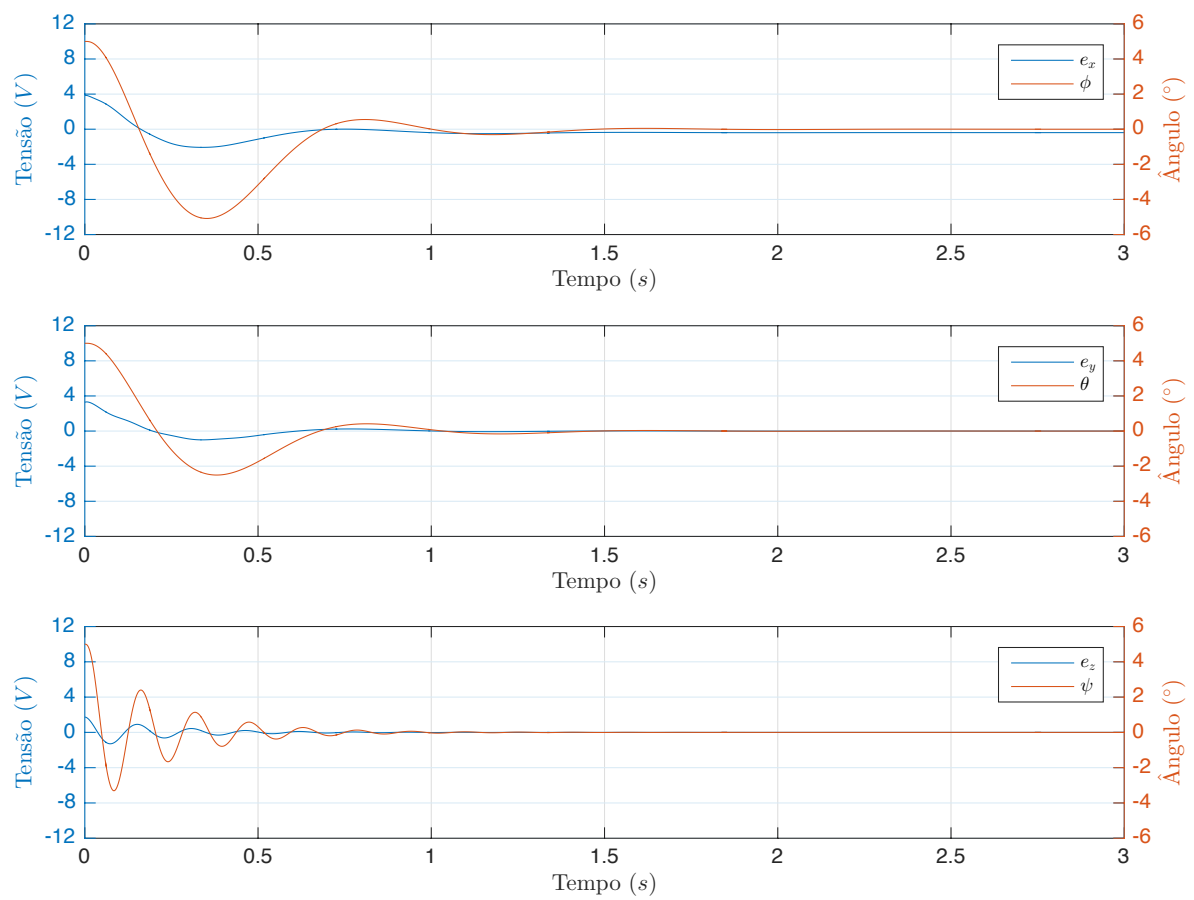

Figura 4.27: Resultado da simulação 8

Analisando a figura acima percebe-se que o sistema apresentou comportamento muito similar ao sistema sem os motores elétricos, com uma dinâmica mais rápida do que a simulação anterior.

\subsection{Controlador final obtido}

O controlador final obtido pode ser observado na Figura 4.28. 


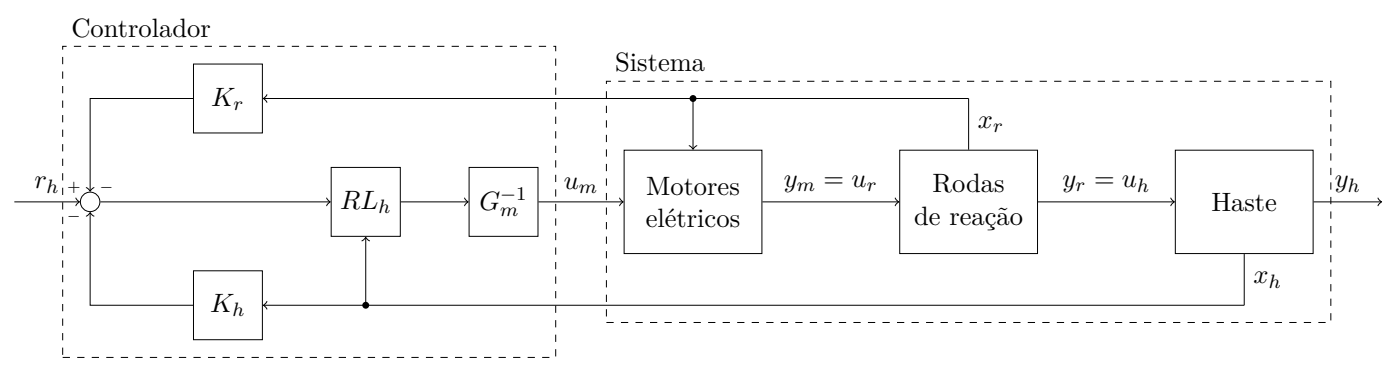

Figura 4.28: Diagrama de blocos do sistema composto pela haste, rodas de reação e motores elétricos com realimentação linearizante da haste, realimentação de estados da haste, realimentação de estados das rodas de reação e compensador de ganhos dos motores elétricos

Onde:

$$
\begin{gathered}
K_{h}=\left[\begin{array}{cccccc}
-1,68 & 0 & 0 & -0,20 & 0 & 0 \\
0 & -1,68 & 0 & 0 & -0,20 & 0 \\
0 & 0 & -1,00 & 0 & 0 & -0,04
\end{array}\right] \\
K_{h}=\left[\begin{array}{ccc}
2,22 & 0 & 0 \\
0 & 2,22 & 0 \\
0 & 0 & 2,22
\end{array}\right] \\
G_{m}^{-1}=\left[\begin{array}{ccc}
20 & 0 & 0 \\
0 & 20 & 0 \\
0 & 0 & 20
\end{array}\right]
\end{gathered}
$$

Já o bloco $R L_{h}$ é a realimentação linearizante não-linear definida na Seção 4.2.1.

Note que apenas os estados da haste e das rodas de reação estão sendo utilizados para controle. Pela dinâmica dos motores elétricos ser extremamente rápida se comparada as da haste e das rodas, a não realimentação de seus estados no controlador não gera impactos significativos. E conforme foi verificado na simulação 8, tal controlador apresentou resultados satisfatórios.

Entretanto, algo que não foi análisado a fundo é a vantagem da realimentaçnao linearizante não-linear $R L_{h}$. Para isso, foram realizadas duas novas simulações: uma sem a realimentação linearizante e outra com.

\subsubsection{Simulações}

\section{Simulação 9}

Para destacar o efeito da realimentação linearizante foram assumidas como condições iniciais da posição e orientação da haste ângulos de $50^{\circ}$, bem distantes do ponto de equilíbrio do sistema: 


$$
x_{h 0}=\left[\begin{array}{c}
0,8726 \\
0,8727 \\
0,8727 \\
0 \\
0 \\
0
\end{array}\right]
$$

Dessa forma, o sistema não-linear não pode ser aproximado ao sistema com linearização padrão, o que aumenta a relevância da realimentação linearizante no controlador.

Inicialmente foi simulado o sistema sem a realimentação linearizante e o resultado obtido pode ser observado na Figura 4.29.
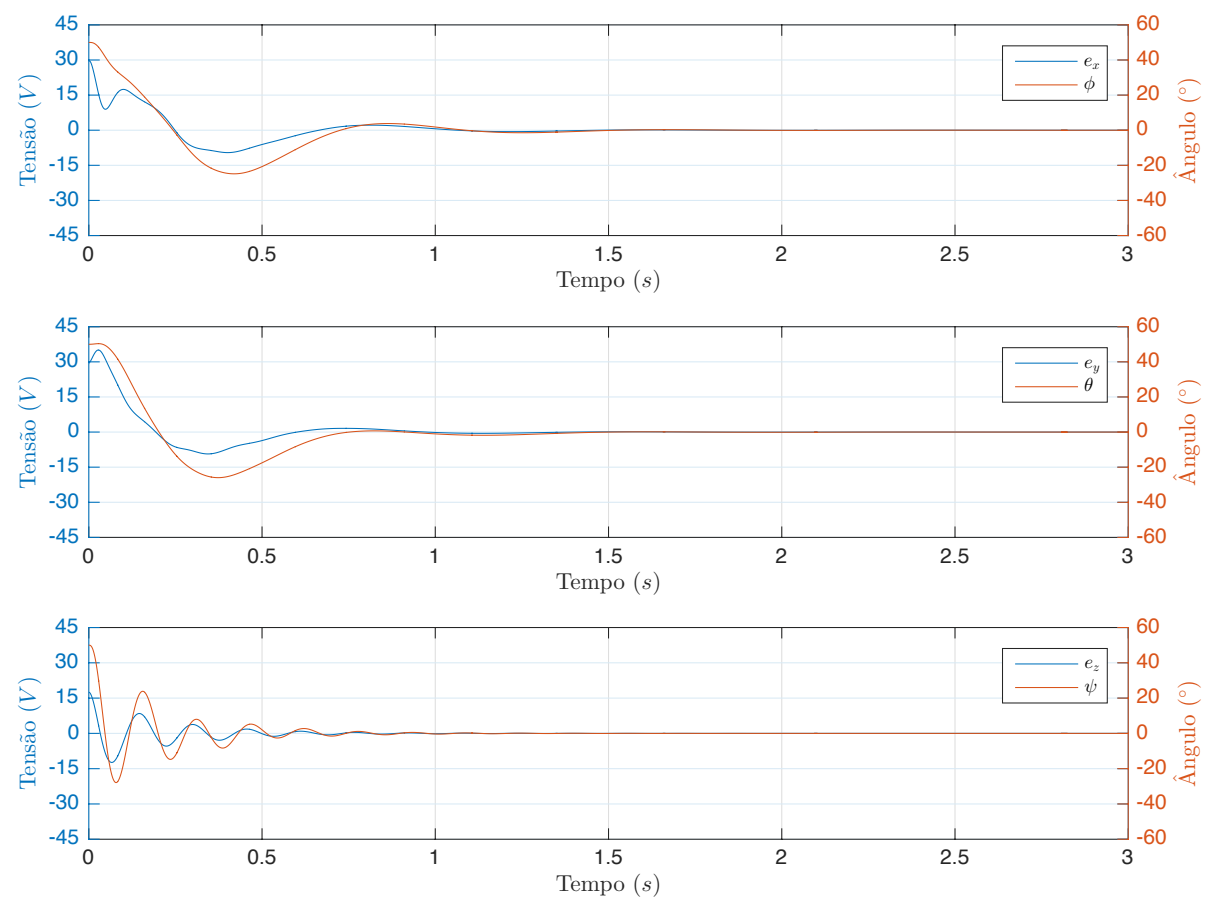

Figura 4.29: Resultado da simulação 9

Conforme pode ser observado na figura acima, o controlador sem realimentação linearizante estabilizou o sistema. Entretanto, a saída do sistema apresentou uma trajetória não muito uniforme até a estabilização.

\section{Simulação 10}

Em seguida foi simulado o sistema com a realimentação linearizante e o resultado obtido pode ser observado na Figura 4.30. 

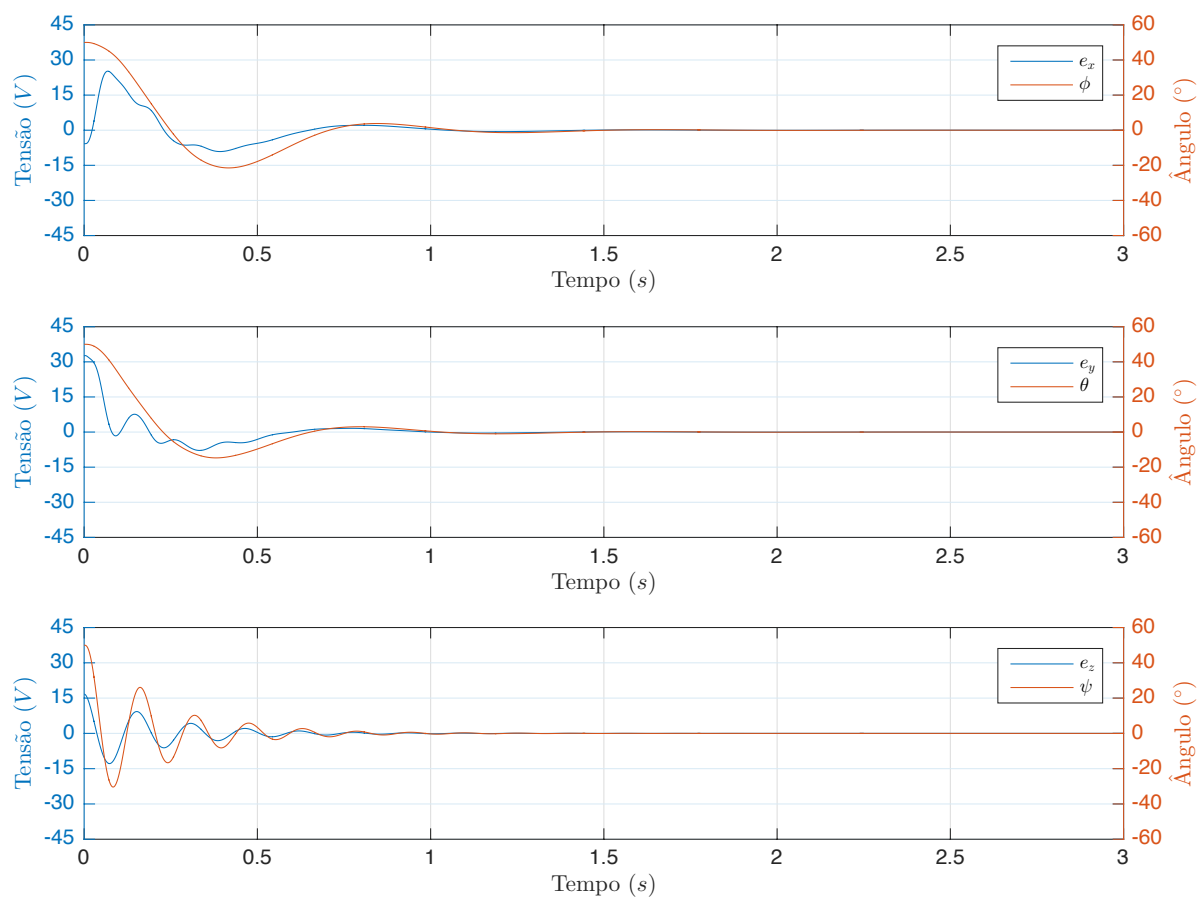

Figura 4.30: Resultado da simulação 10

Nessa nova simulação o sistema também se estabilizou e a saída aparentemente apresentou uma tajetória mais uniforme. Entretanto, o resultado final de ambas as simulações é muito similar.

Para destacar melhor as diferenças entre essas duas simulações (sem e com realimentação linearizante), ambos os resultados serão exibidos nas mesmas figuras.

Na Figura 4.31 tem-se um comparativo entre as entradas dessas duas simulações. 

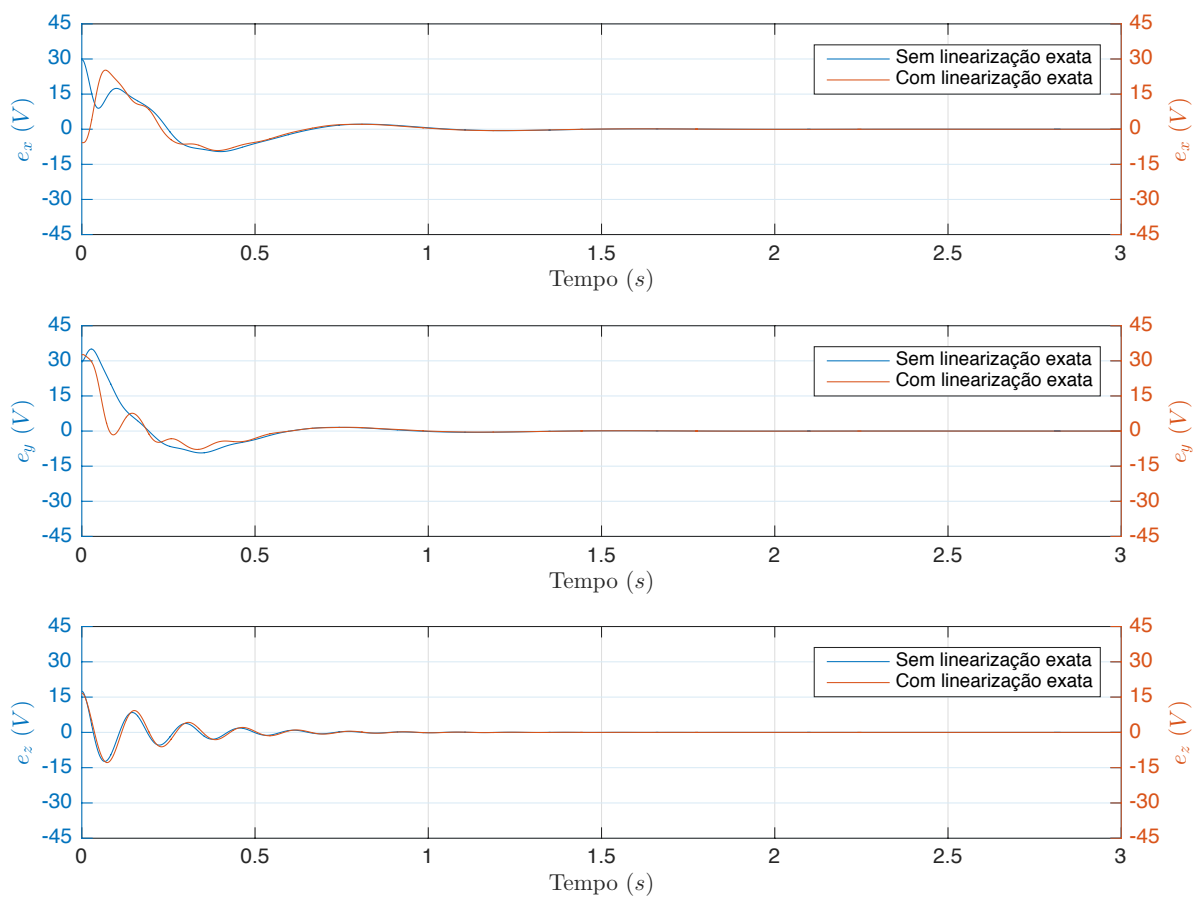

Figura 4.31: Comparação das entradas nas simulações com e sem a realimentação linearizante

Já na Figura 4.32 tem-se um comparativo entre as saídas dessas duas simulações. 

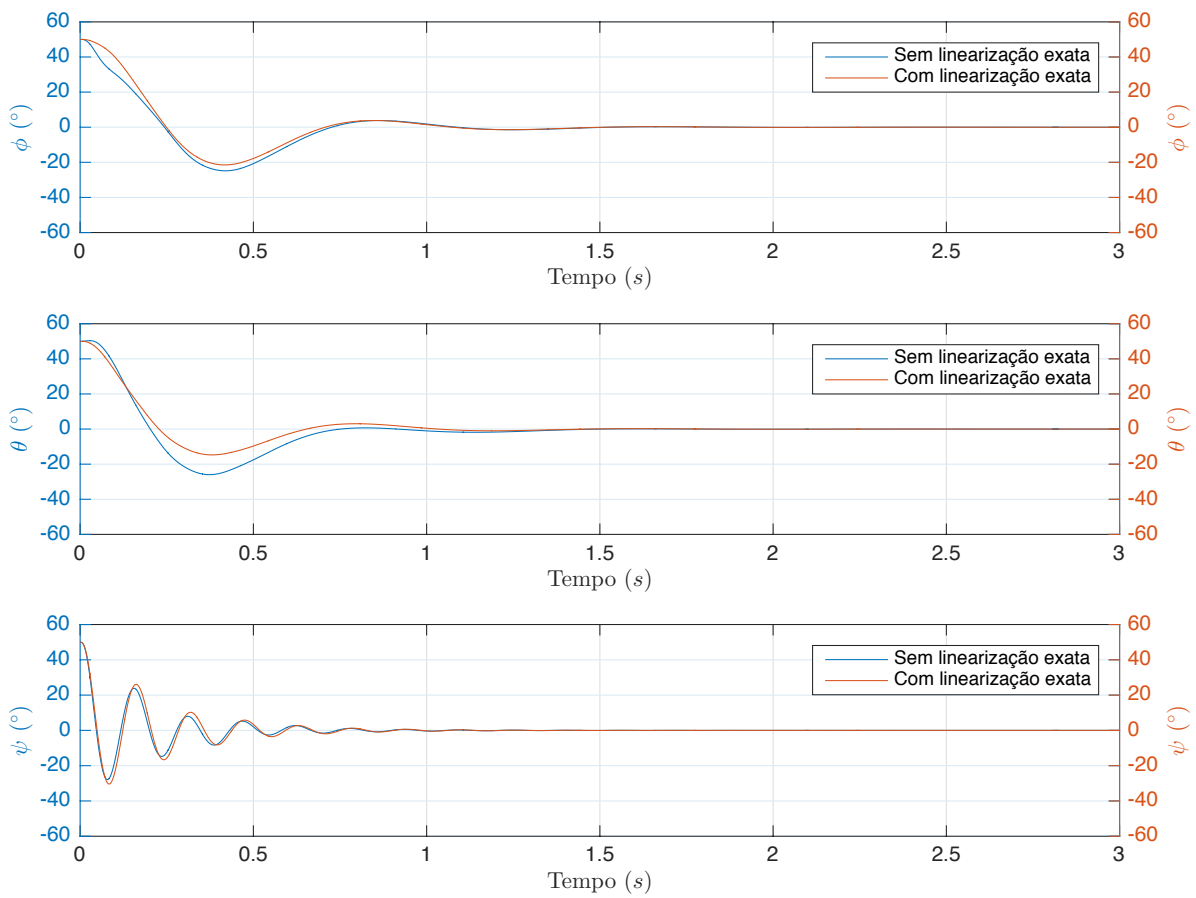

Figura 4.32: Comparação das saídas nas simulações com e sem a realimentação linearizante

Conforme é possível observar, as entradas da simulação sem realimentação linearizante apresenta picos lijeiramente superiores aos da simulação com realimentação linearizante. Além disso, observando as saídas, novamente a simulação sem realimentação linearizante apresentou picos lijeiramente superiores, além de uma trajetória menos uniforme.

Entretanto, apesar dessas pequenas diferenças, ambas as simulações apresentaram resultados muito similares. Isso significa que ainda que a o controlador com realimentação linearizante seja melhor, o controlador sem a realimentação linearizante não deixa a desejar. 


\section{Capítulo 5}

\section{Conclusão}

Um tipo diferente de pêndulo invertido controlado por rodas de reação foi descrito e modelado por meio das equações de Euler-Lagrange, das leis de Newton e das leis de Kirchhoff. O sistema foi, então, sub-dividido em três sub-sistemas: a haste, as rodas de reação e os motores elétricos. Cada um desses sub-sistemas foi analisado individualmente e, em seguida, seguiu-se com o projeto do controlador.

Inicialmente, apenas a dinâmica da haste foi considerada e o sistema foi então linearizado sob duas técnicas conhecidas na literatura: linearização padrão e linearização exata. A linearização padrão foi utilizada para as etapas de projeto do controlador, enquanto que a linearização exata foi utilizada nas simulações. Uma realimentação dos estados da haste foi projetada com base nos conceitos de controle ótimo. Em seguida, a dinâmica das rodas de reação foi inserida no sistema. Uma realimentação de estados adicional, dessa vez dos estados das rodas de reação, foi projetada a partir de algumas simplificações e do critério de estabilidade de Routh. Por fim, a dinâmica dos motores elétricos foi considerada no sistema. Um compensador de ganhos foi inserido no controlador assumindo que os motores elétricos podem ser aproximados a transdutores de torque de ganho constante.

O teste e a validação do controlador foram realizados através de simulações dinâmicas, nas quais todas as simplificações assumidas para o projeto não estavam presentes. O intuito do projeto de controle era obter um comportamento da haste próximo ao esperado pela linearização padrão da mesma. Isso foi obtido pela combinação da linearização exata da haste com a inclusão de uma pequena realimentação da velocidade das rodas de reação para a consequente estabilização da velocidade das mesmas.

Foi possível concluir que, quando o pêndulo está próximo de seu ponto de equilíbrio, a linearização exata se equivale a linearização padrão e, portanto, o controlador desenvolvido para o sistema com linearização padrão pode ser implementado no sistema com linearização exata. Além disso, uma realimentação de estados das rodas de reação pequena garante a estabilidade do sistema como um todo sem alterar drasticamente a dinâmica da haste. Por fim, como a dinâmica dos motores elétricos é extremamente rápida se comparada com a dinâmica da haste e das rodas de reação, a simplificação dos mesmos como transdutores de torque de ganho constante traz grandes facilidades 
no projeto do controlador sem gerar grandes implicacões nas simulações.

Quanto a trabalhos futuros, há alguns aprimoramentos que poderiam ser considerados. O primeiro seria projetar um observador de estados para o sistema. Apesar de todos os estados do sistema em estudo poderem ser facilmente obtidos por meio de uma $\mathrm{IMU}^{1}$, tal observador não desempenharia a função de estimar aqueles que não estão sendo medidos, mas sim de operar como um filtro de Kalman. Dessa forma, o controlador poderia compensar não só erros aditivos, conforme foi visto neste trabalho, mas também erros aleatórios e assim aprimorar sua robustez. Um segundo aprimoramento seria construir um protótipo do sistema. Embora, hoje em dia, seja possível modelar e representar fielmente sistemas dinâmicos complexos através de modelos matemáticos, nem sempre o que é esperado na teoria acontece na prática. A única forma de realmente validar o controlador projetado seria por meio da construção de um protótipo.

\footnotetext{
${ }^{1}$ Dispositivo eletrônico composto por acelerômetros, giroscópios e magnetrômetros, em inglês "Inertial Measurement Unit"
} 


\section{Referências Bibliográficas}

Aristotle (1984). The Complete Works of Aristotle. Princeton University Press.

Block, D. J.; Astrom, K. L. and Spong, M. W. (2007). The Reaction Wheel Pendulum. Morgan \& Claypool.

Craig, J. J. (2005). Introduction to Robotics. Pearson.

Dion, C. (2008). Solution of Lagrange's and Hamilton's equations of motion for a symmetric top. International Journal of Non-Linear Mechanics, pages 794-800.

Dion, C. and Nyln, M. (2013). Solution of Lagrange's and the discrete Lagrange's equations of motion for a symmetric top. Computer Methods in Applied Mechanics and Engineering, pages 49-66.

Fliess, M.; Lévine, J. M. P. and Rouchon, P. (1995). Flatness and defect of nonlinear systems. International Journal of Control, pages 1327-1361.

Friedland, B. (2005). Control System Design: An Introduction to State-Space Methods. Dover Publications.

Galilei, G. (1957). Discoveries and Opinions of Galileo. Anchor.

Goswami, A. (2008). Kinematic and dynamic analogies between planar biped robots and the reaction mass pendulum (RMP) model. IEEE-RAS International Conference on Humanoid Robots, pages 182-188.

Isidori, A. (1995). Nonlinear Control Systems. Springer.

Meirovitch, L. (2003). Methods of Analytical Dynamics. Dover Publications.

Morazzani, I.; Lahr, D. H. D. W. and Ren, P. (2008). Novel Tripedal Mobile Robot and Considerations for Gait Planning Strategies Based on Kinematics. Springer-Verlag, pages 35-48.

Pons, J. L. (2008). Wereable Robots: Biomechatronic Exoskeletons. John Wiley \& Sons.

Seman, P. and Juhás, M. (2011). Swing Up and Stadilization of Reaction Wheel Pendulum. 4th International Conference of Modelling of Mechanical and Mechatronic Systems, pages $47-52$. 
Sheng-Jun, P.; Hai-Tao, S. T. S. and Hong-Xu, M. (2009). A Novel Stability Criterion for Underactuated Biped Robot. IEEE International Conference on Robotics and Biomimetics, pages 1912-1917. 


\section{Apêndice A}

\section{Códigos do MATLAB}

Código A.1: definicao_das_variaveis

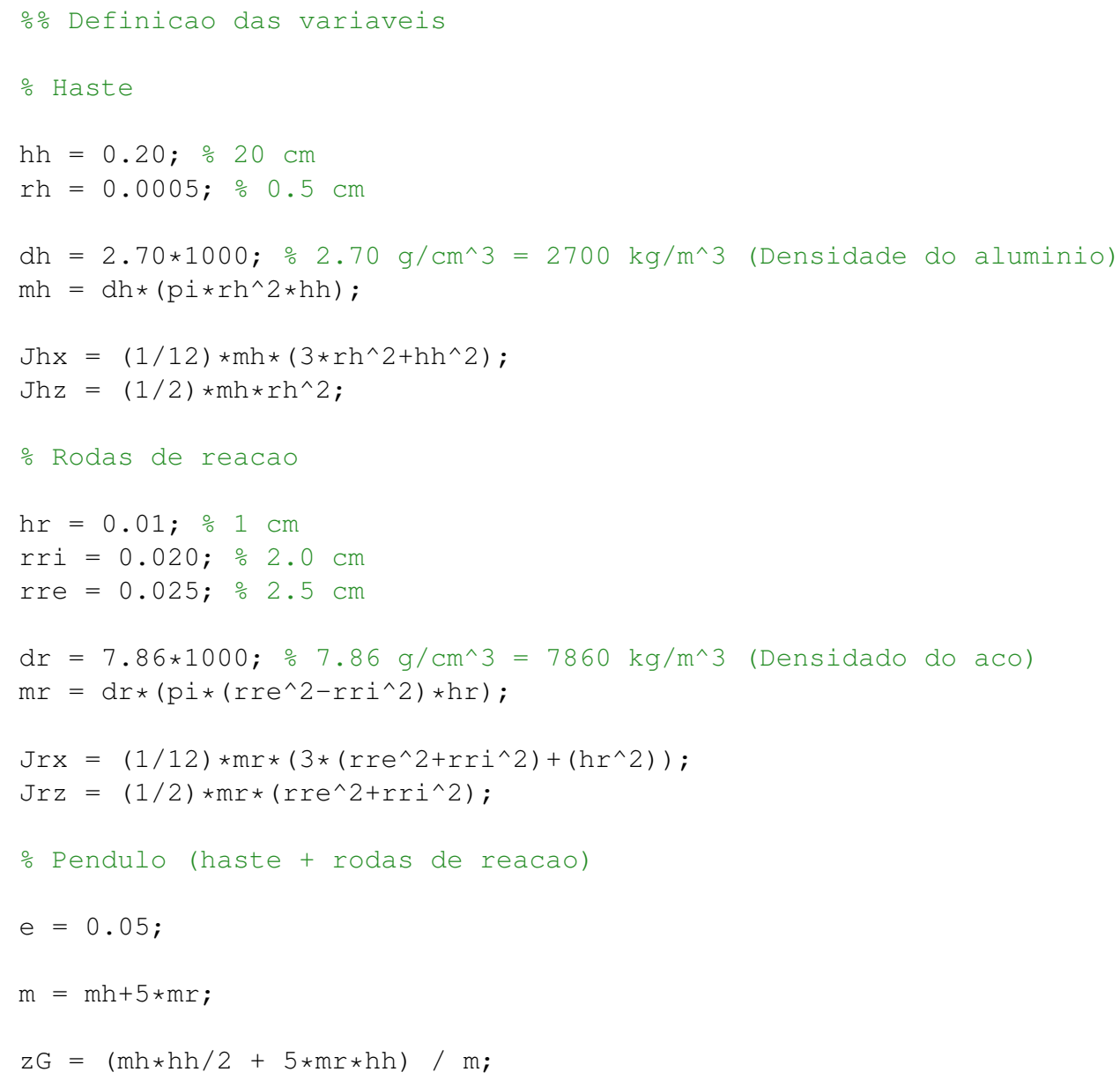




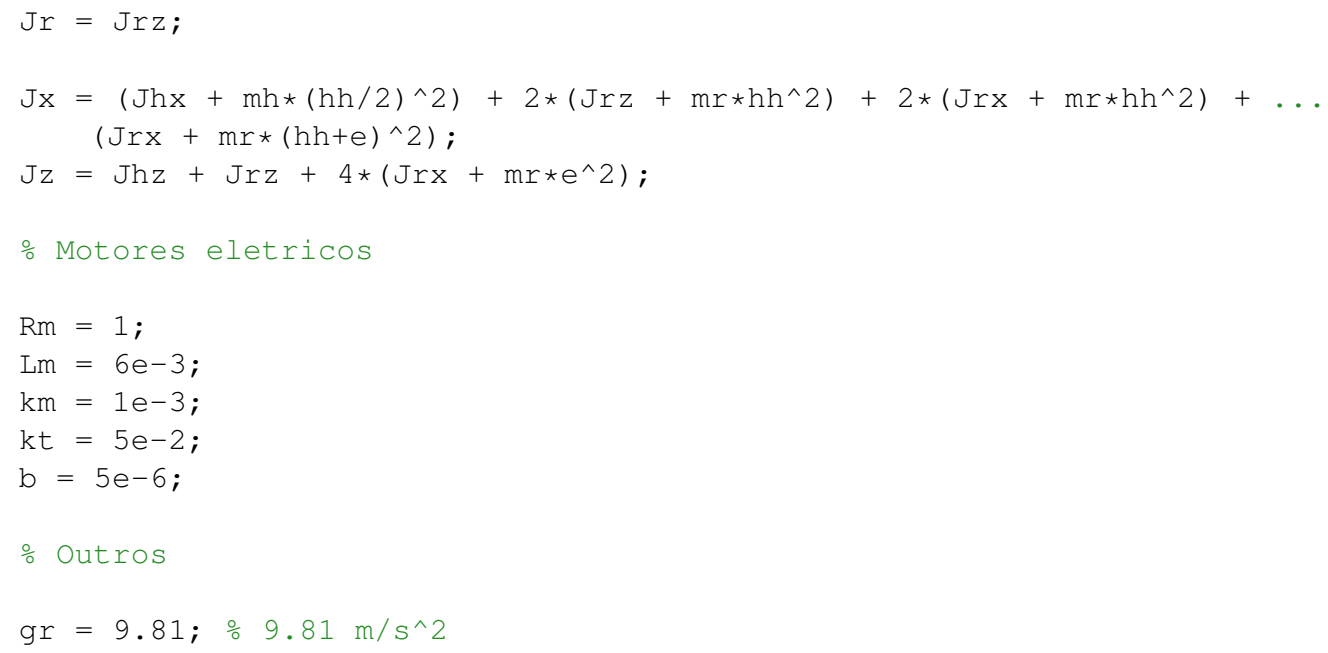

Código A.2: sub_sistema_haste

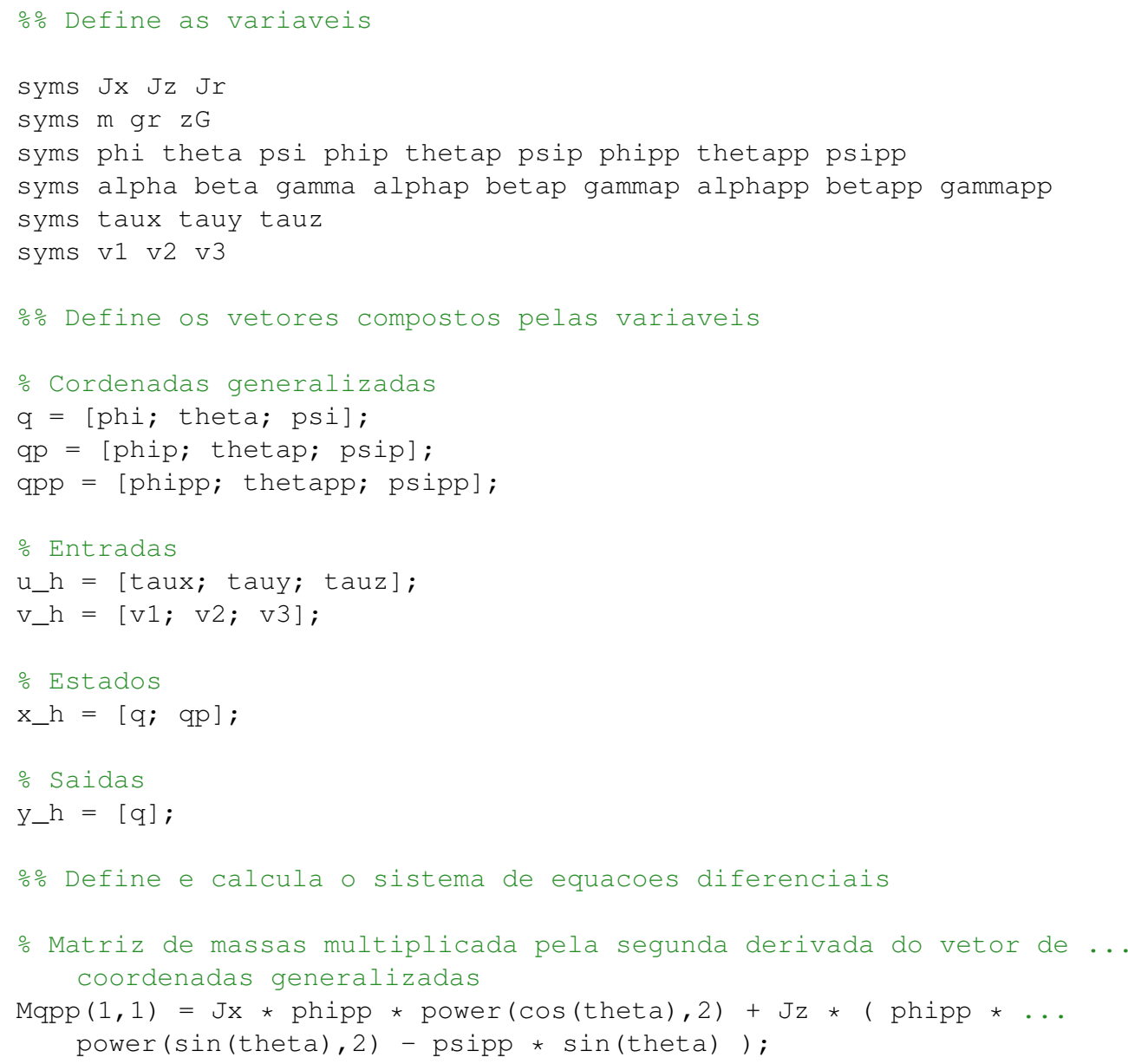




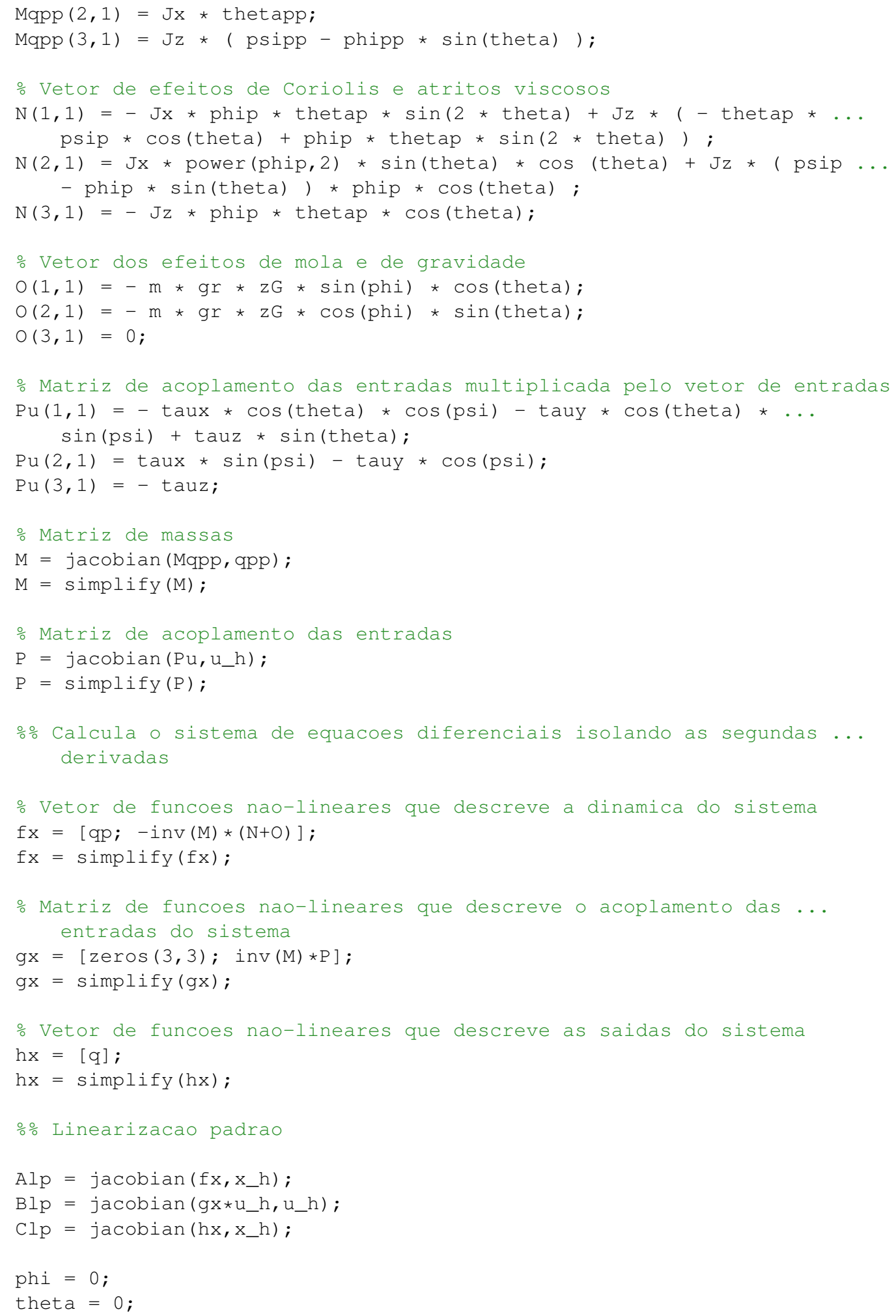




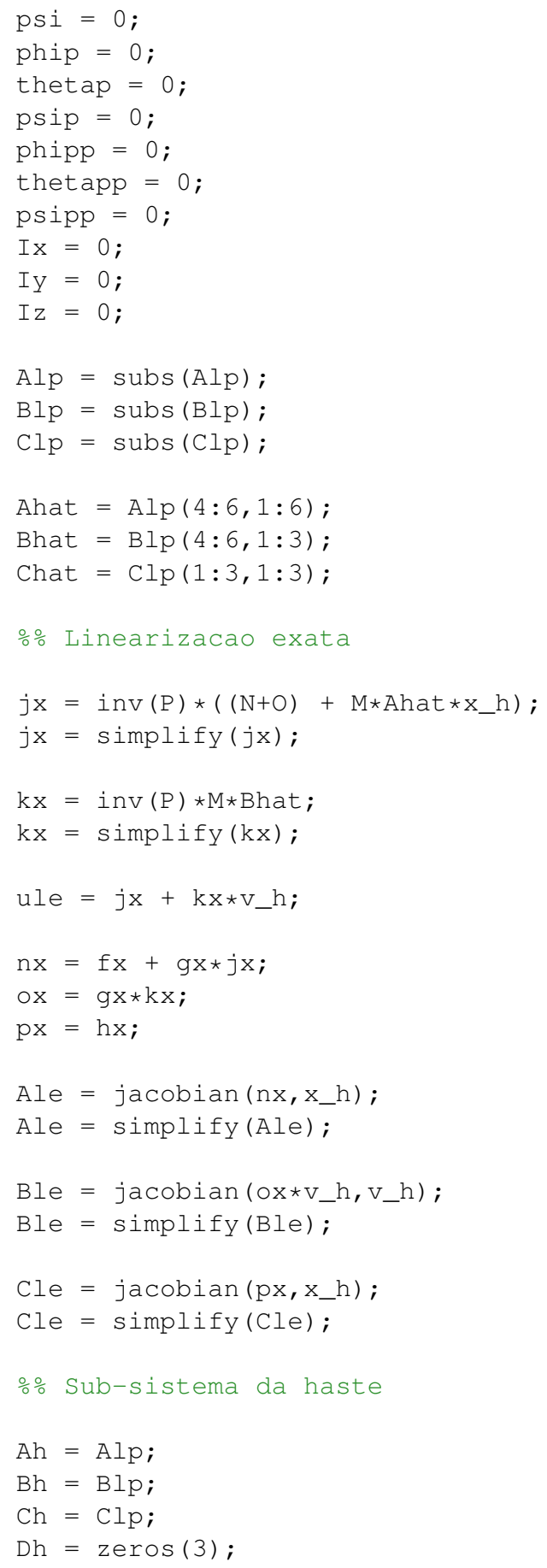

Código A.3: sub_sistema_rodas_de_reacao

$1 \%$ Define as variaveis 


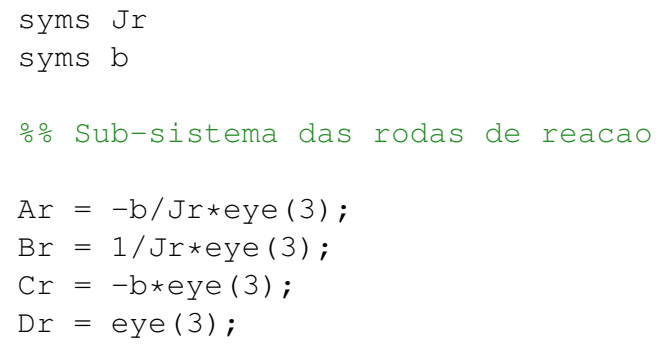

Código A.4: sub_sistema_motores_eletricos

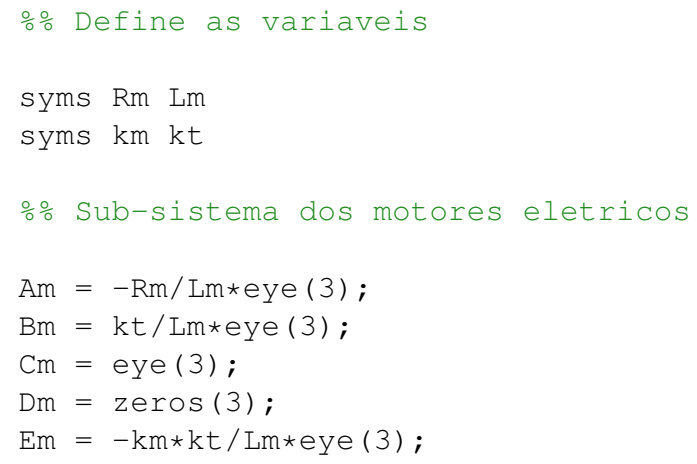

Código A.5: sistema_completo

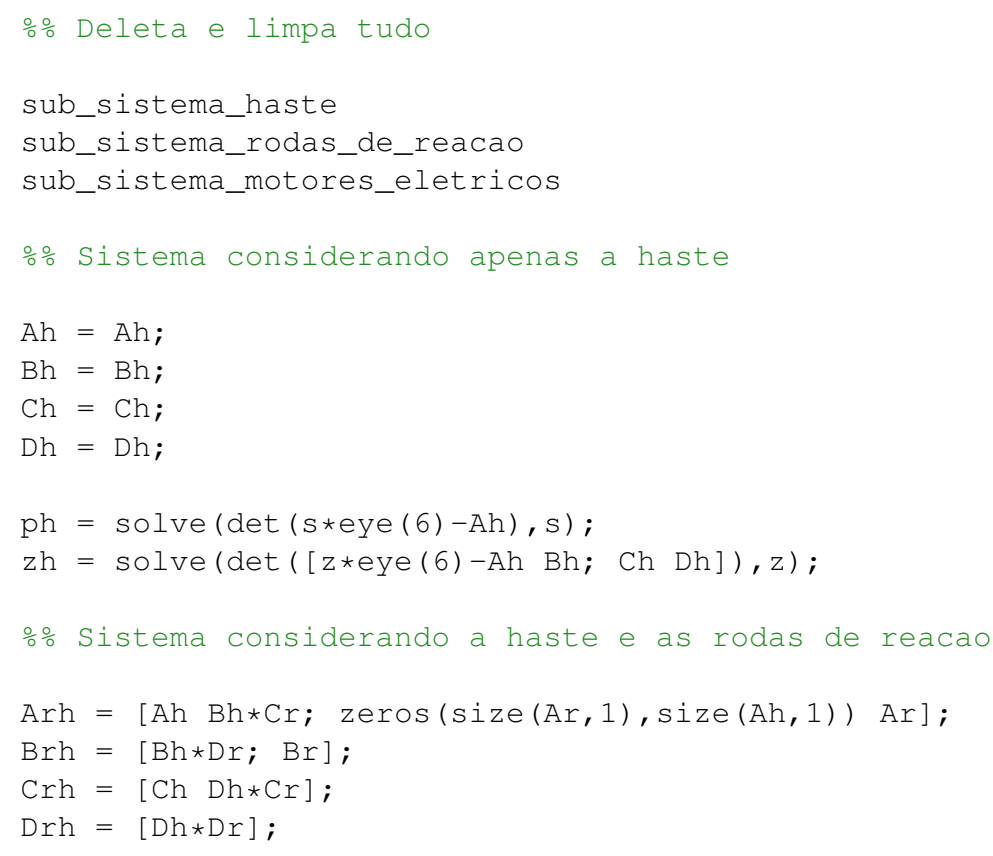




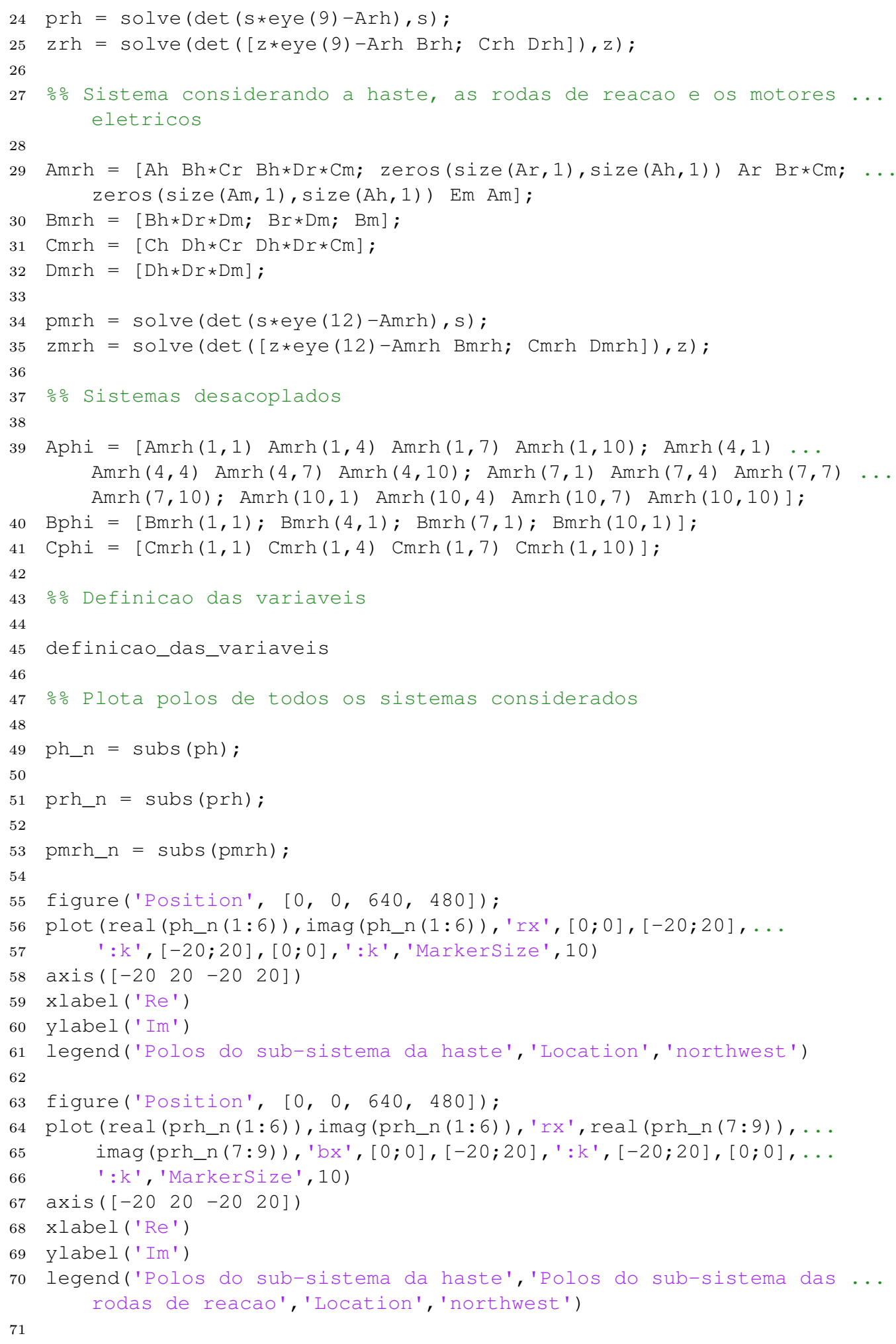




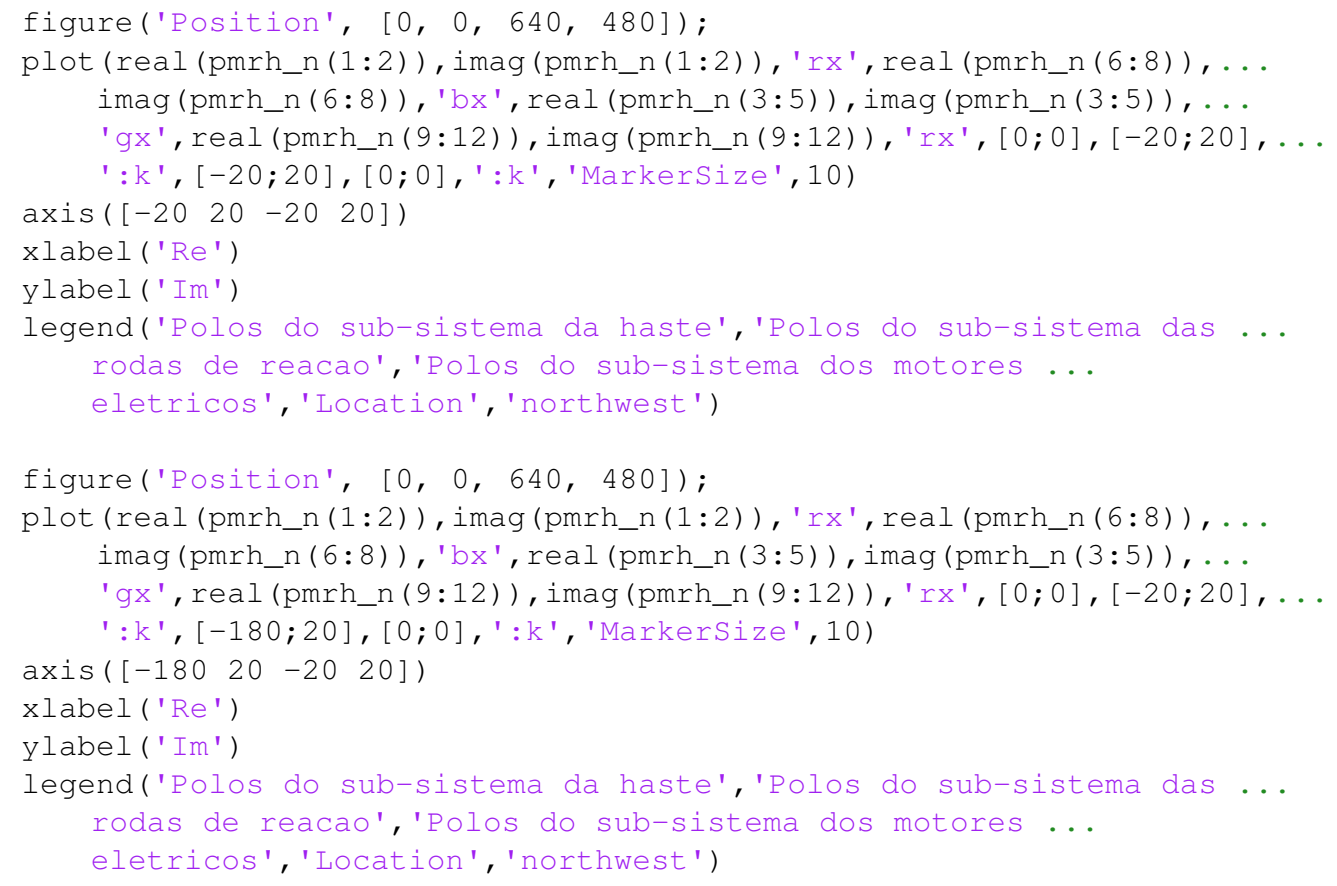

\section{Código A.6: estabilidade_routh}

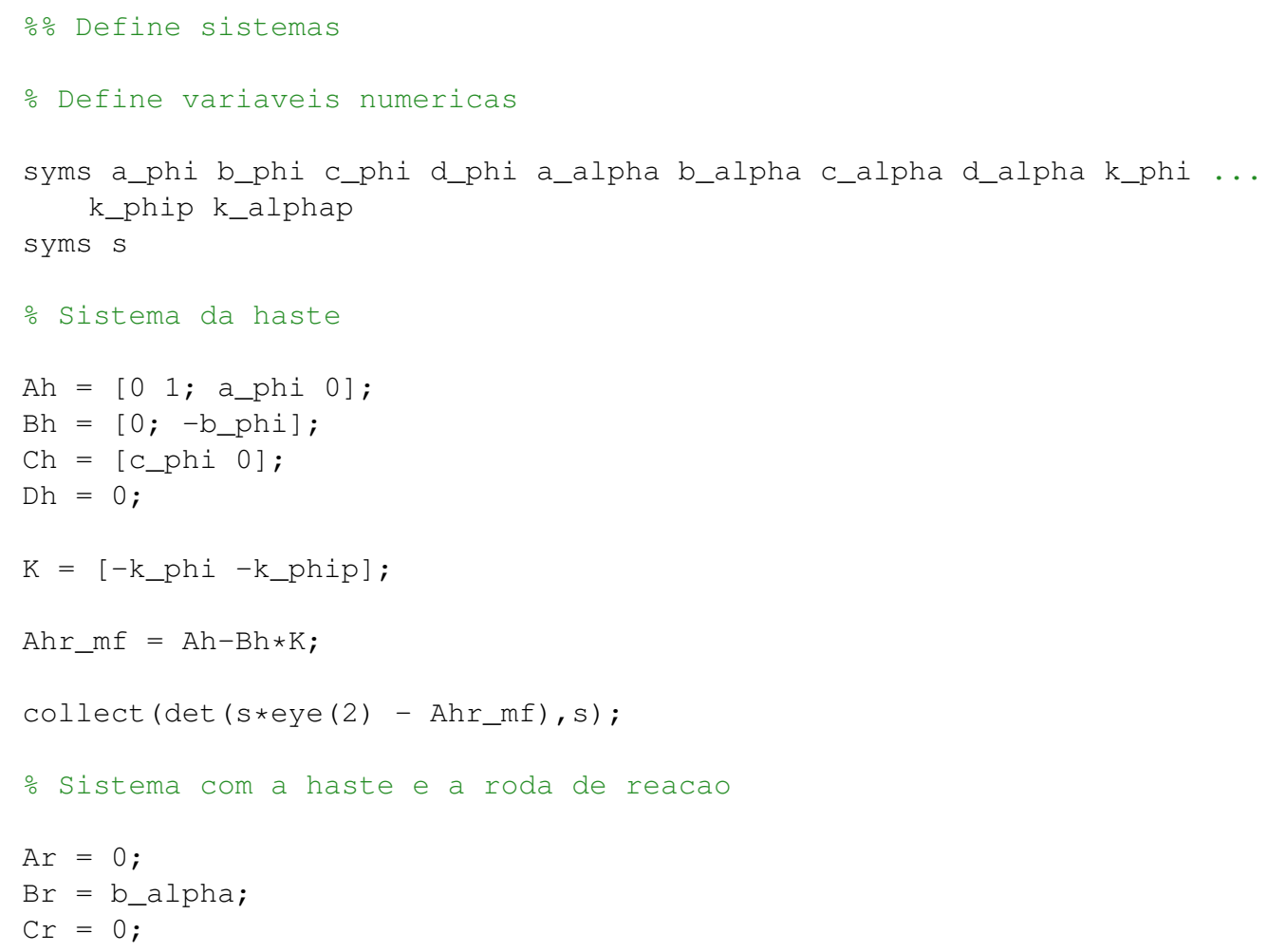




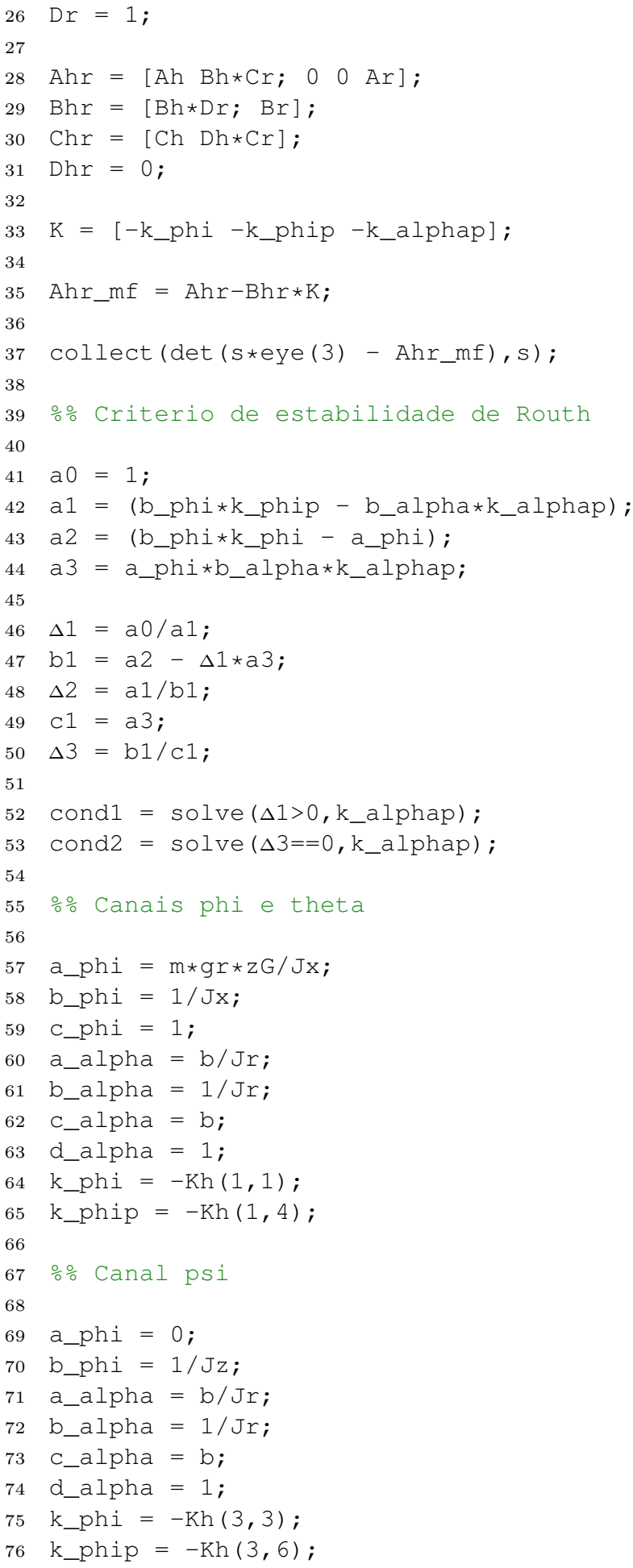


Código A.7: inicializa

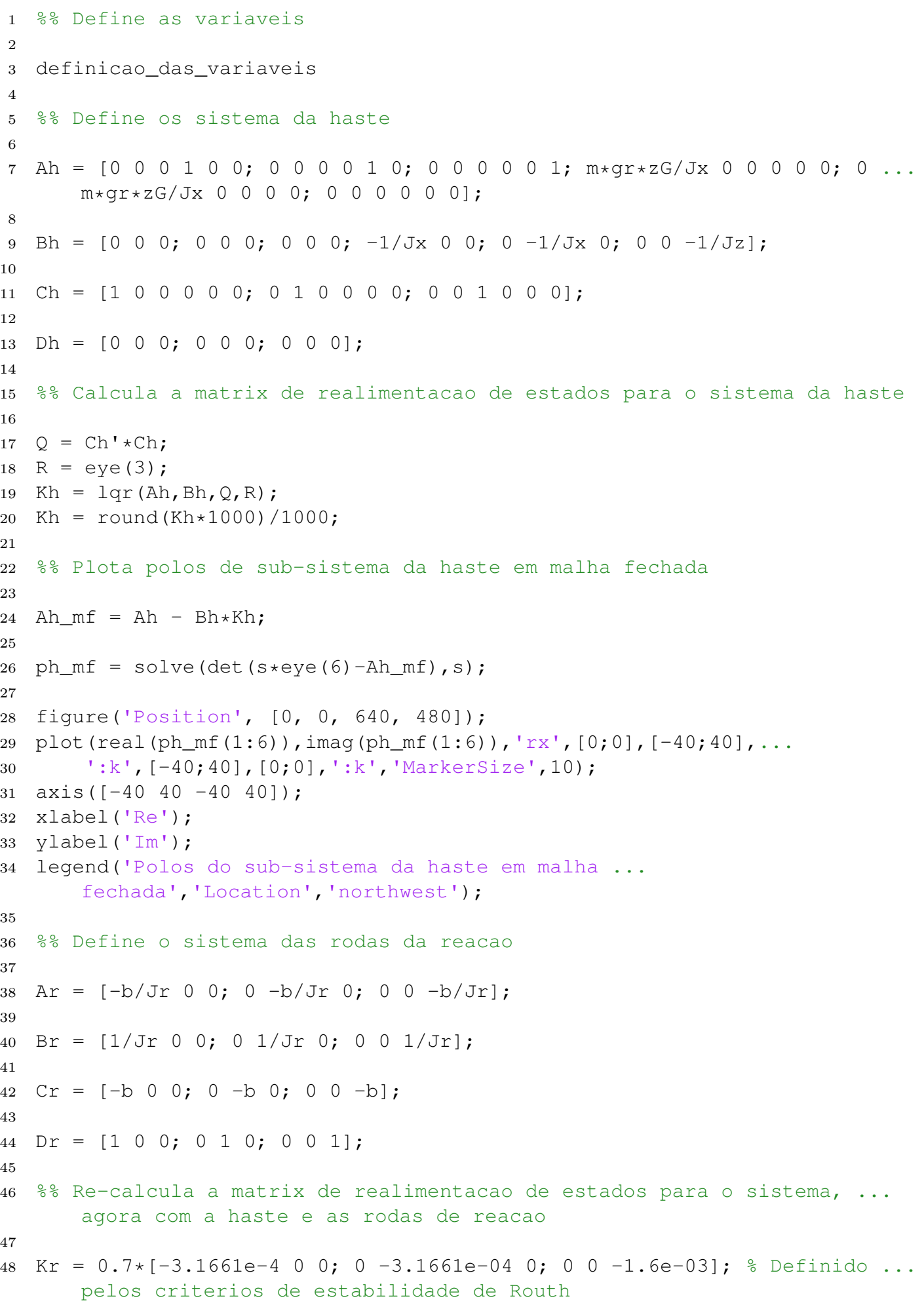




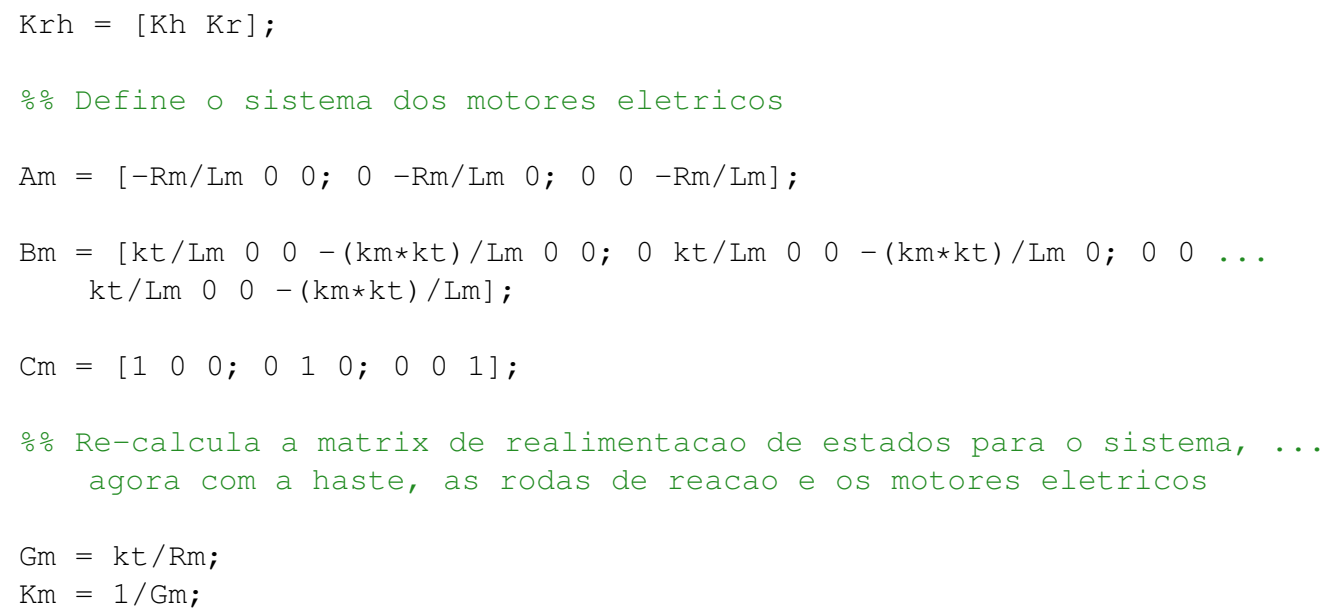

Código A.8: function_f

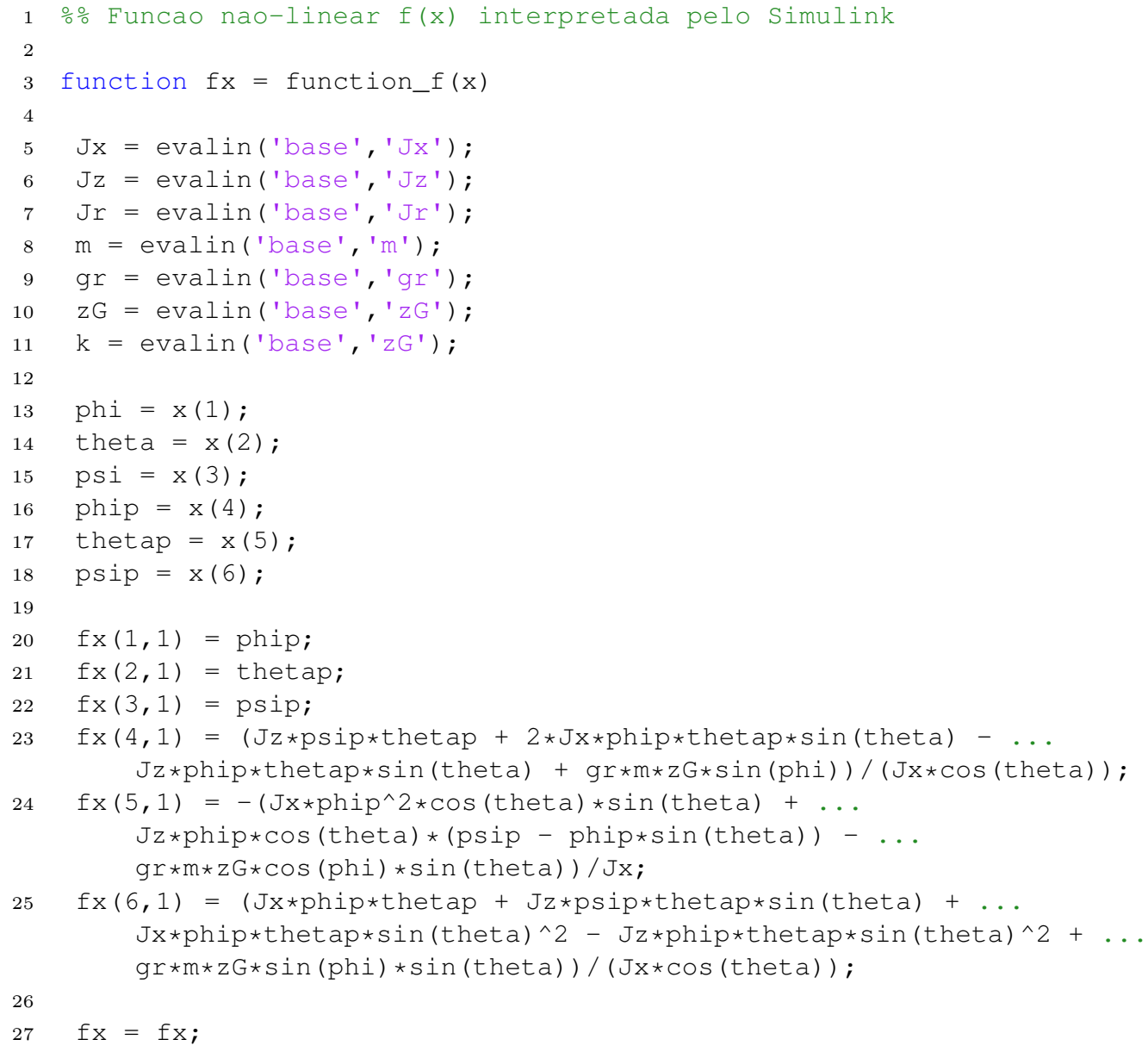


Código A.9: function_g

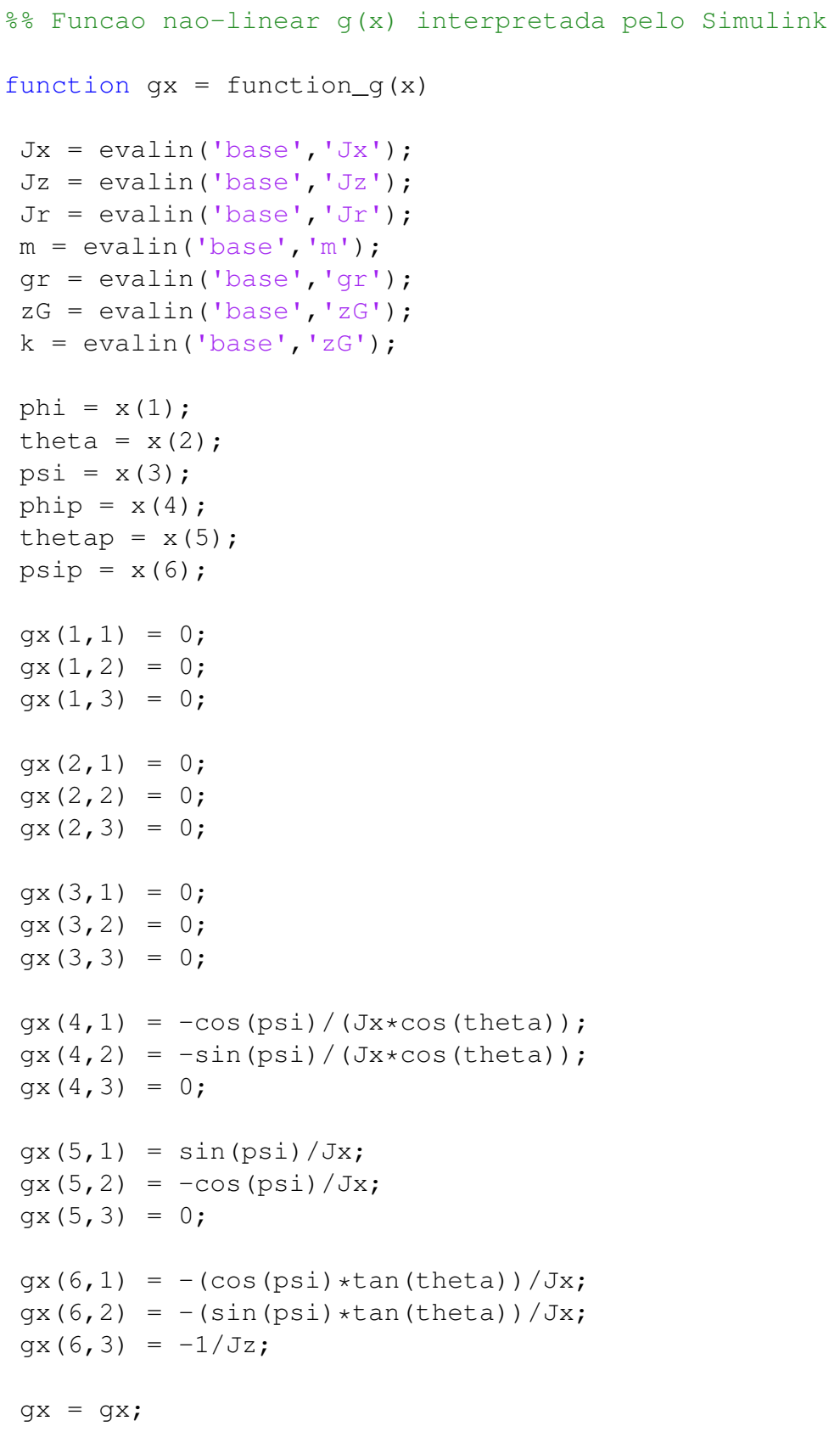

Código A.10: function_h

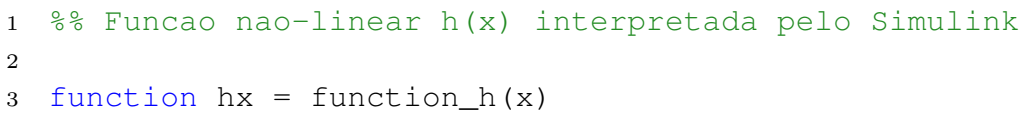




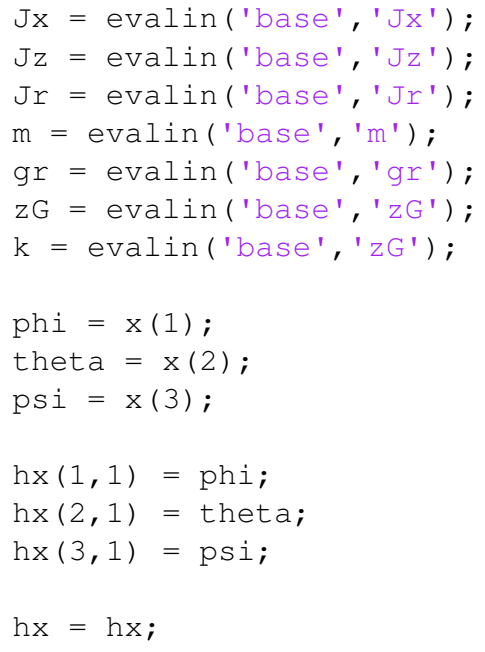

Código A.11: function_

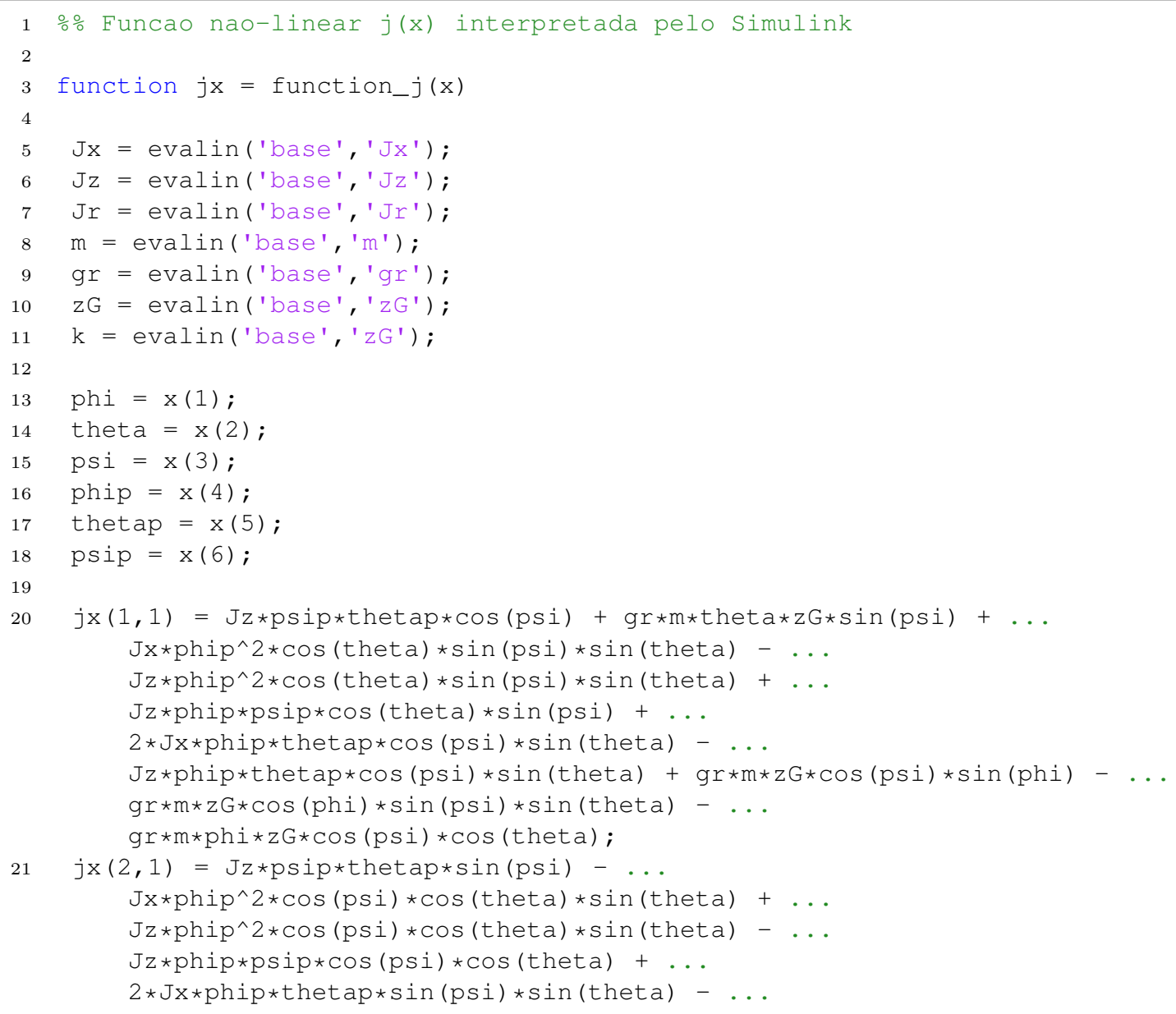




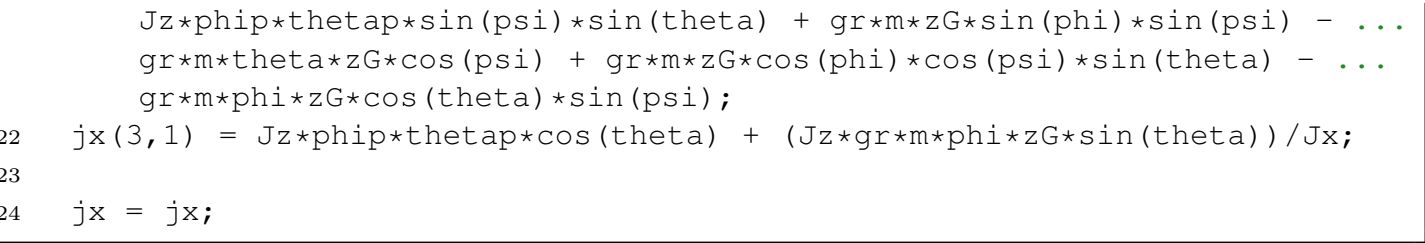

\section{Código A.12: function_k}

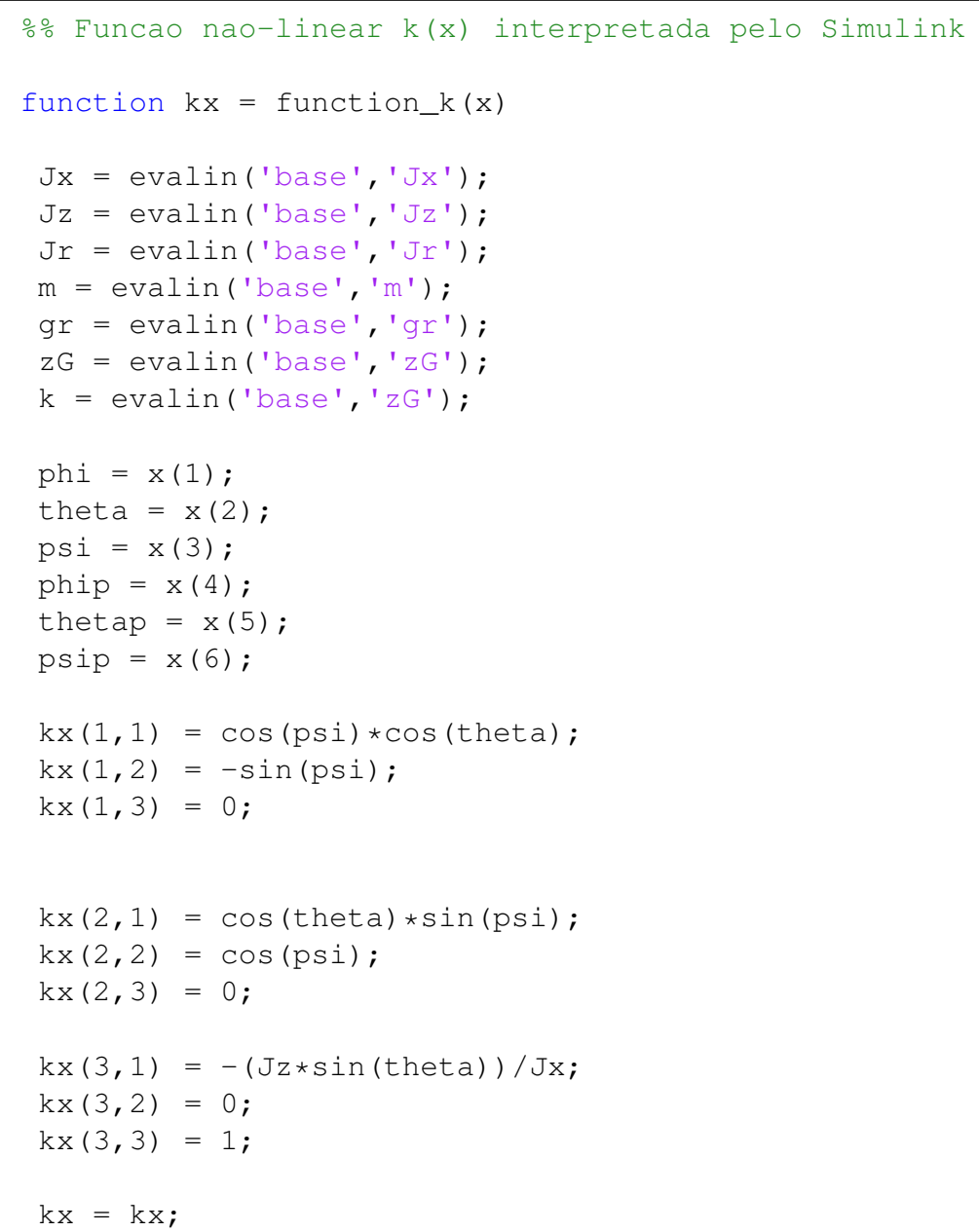

Código A.13: plota_simulacao_haste

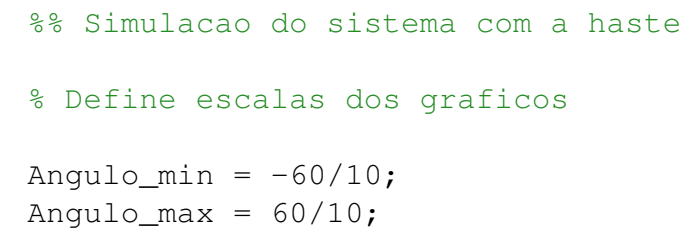




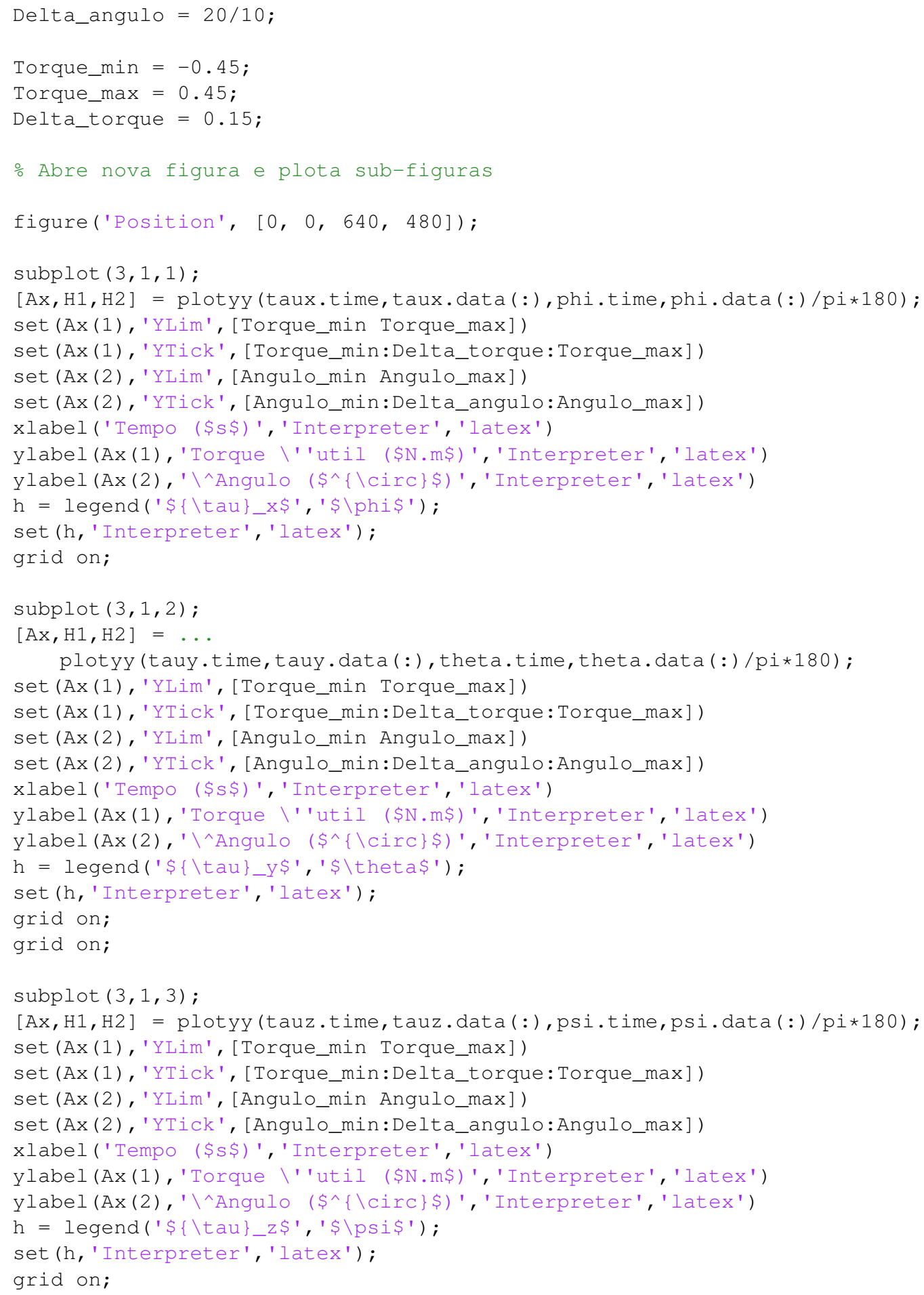


Código A.14: plota_simulacao_haste_rodas

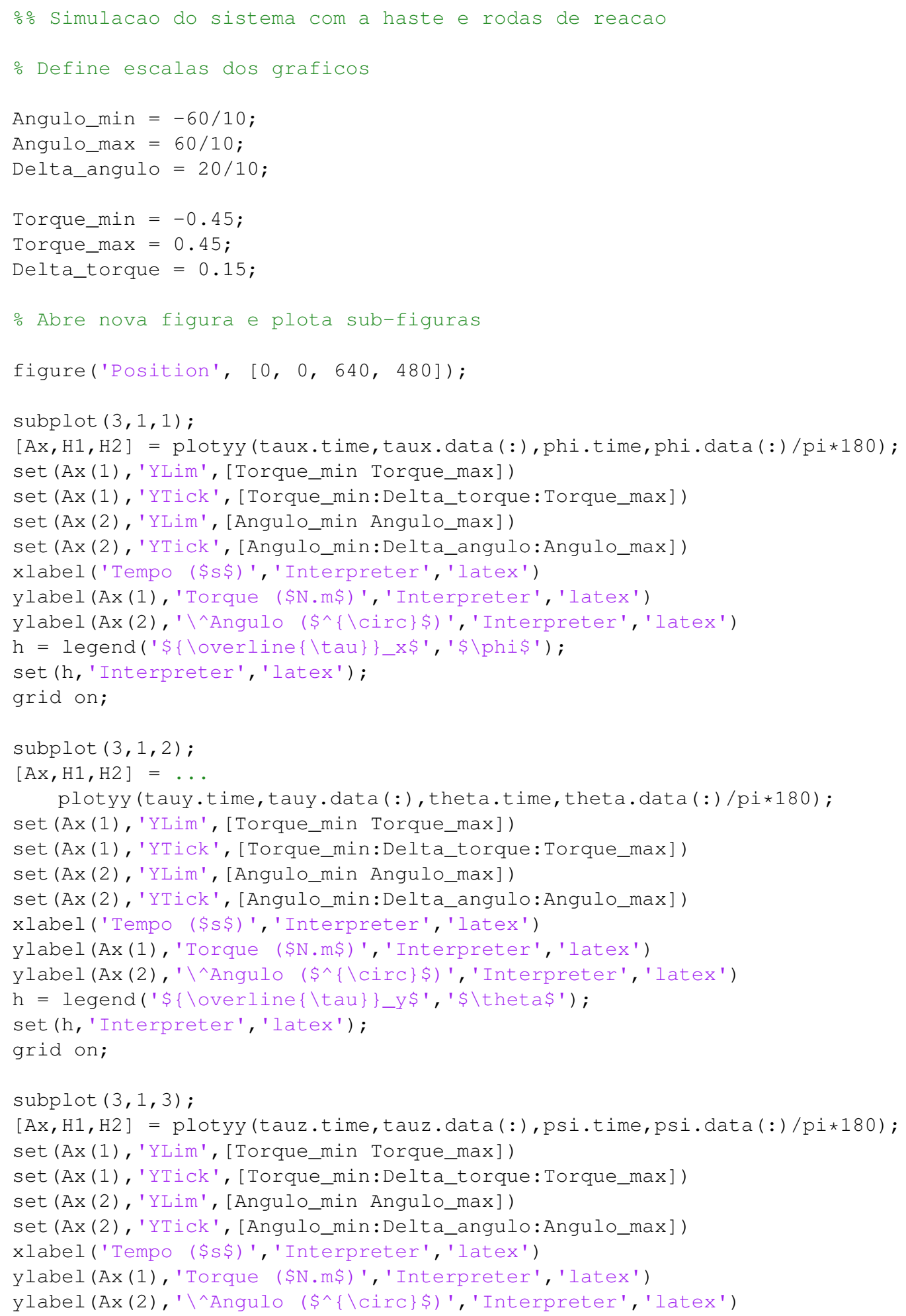


$52 \mathrm{~h}=$ legend('\$\{\overline $\{$ tau $\}$ _z\$', '\$ \psi\$');

53 set (h, 'Interpreter', 'latex');

54 grid on;

Código A.15: plota_simulacao_haste_rodas_motor

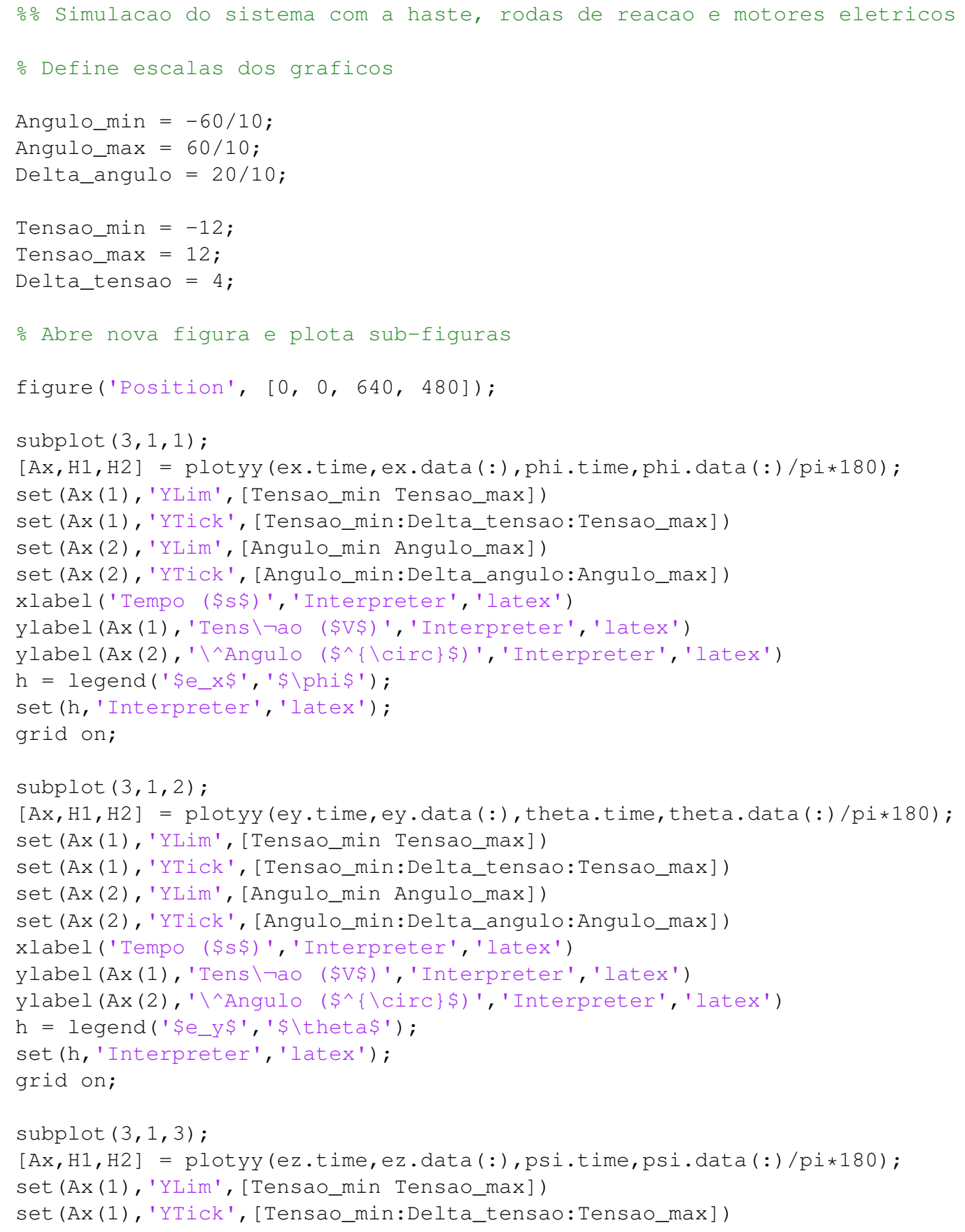


47 set(Ax (2), 'YLim', [Angulo_min Angulo_max])

48 set(Ax (2),'YTick', [Angulo_min:Delta_angulo:Angulo_max])

49 xlabel ('Tempo (\$s\$) ', 'Interpreter', 'latex')

50 ylabel (Ax (1), 'Tens \aao (\$V\$) ', 'Interpreter', 'latex')

51 ylabel (Ax (2), '\^Angulo (\$^\{\Circ\}\$) ', 'Interpreter', 'latex')

$52 \mathrm{~h}=$ legend ('\$e_z\$','\$

53 set (h, 'Interpreter', 'latex');

54 grid on; 